Supporting Information for

\title{
Hydrosilylation of Aldehydes and Ketones Catalyzed by a 2-Iminopyrrolyl Alkyl-manganese(II) Complex
}

\author{
Tiago F. C. Cruz, * Luís F. Veiros, Pedro T. Gomes* \\ Centro de Química Estrutural, Departamento de Engenharia Química, Instituto Superior \\ Técnico, Universidade de Lisboa, Av. Rovisco Pais 1, 1000-049 Lisboa, Portugal. \\ *Corresponding Authors; E-mails: carpinteirocruz@ tecnico.ulisboa.pt; \\ pedro.t.gomes@tecnico.ulisboa.pt
}




\section{Table of contents}

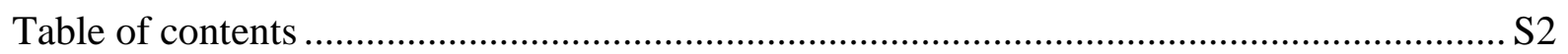

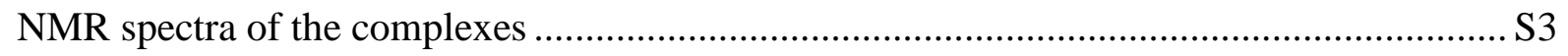

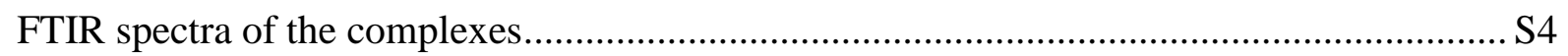

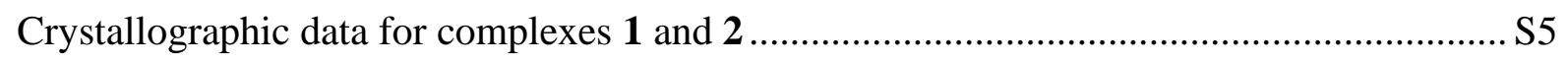

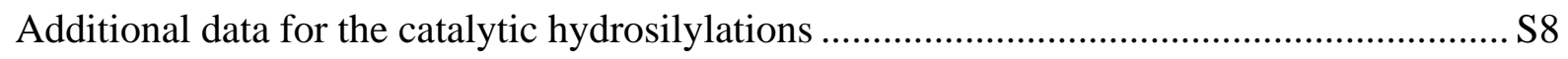

NMR data for the conversion of the aldehydes and ketones to the corresponding silanes

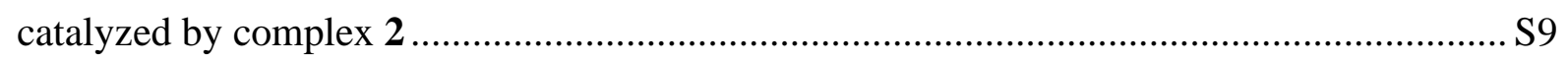

NMR data of the alcoholic products obtained from hydrolysis of the silanes .................... S19

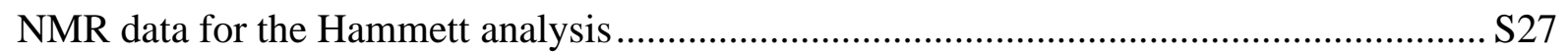

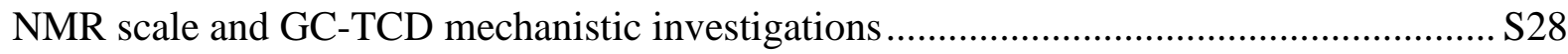

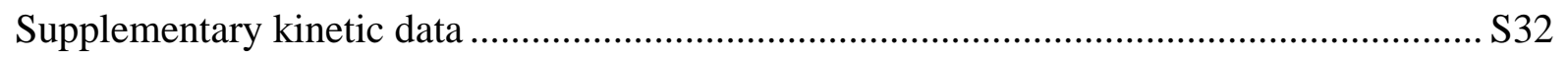

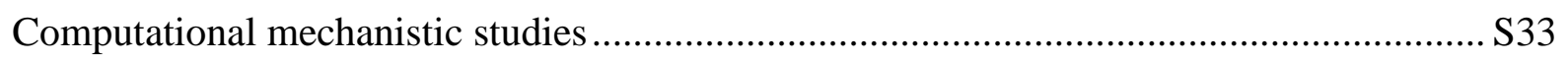

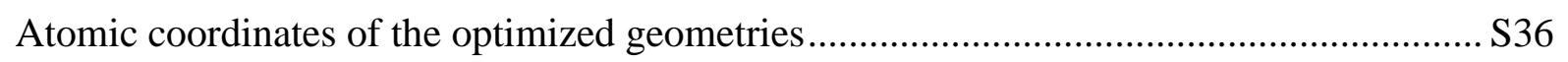




\section{NMR spectra of the complexes}

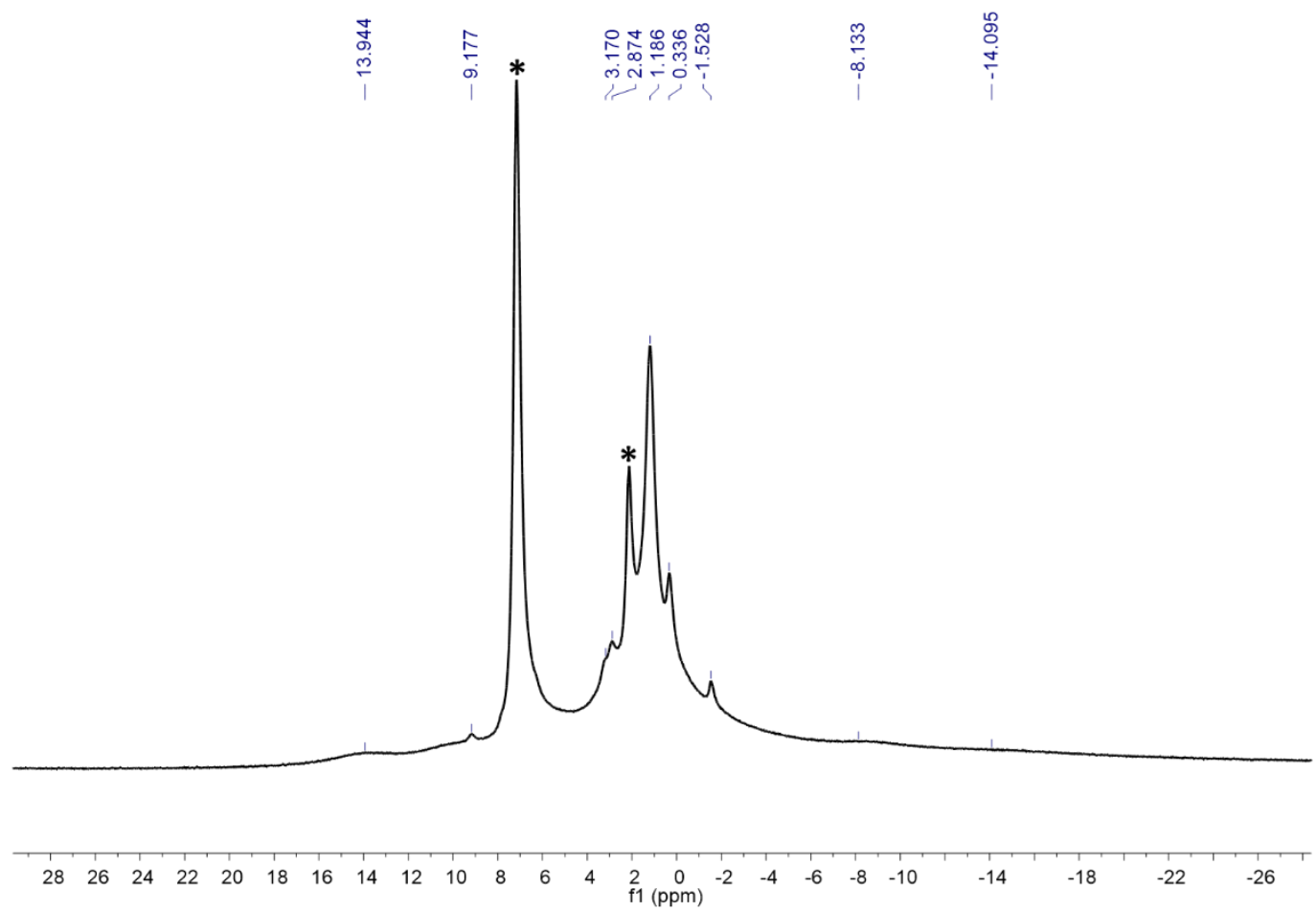

Figure S1 ${ }^{1} \mathrm{H}$ NMR spectrum ( $\left.300 \mathrm{MHz}, \mathrm{C}_{6} \mathrm{D}_{6}\right)$ of complex 1 ( $\left.c a .0 .1 \mathrm{M}\right)$. The asterisk denotes the protio-residual resonance of $\mathrm{C}_{6} \mathrm{D}_{5} \mathrm{H}$ and residual toluene. The resonance at $2.132 \mathrm{ppm}$ corresponds to residual toluene.

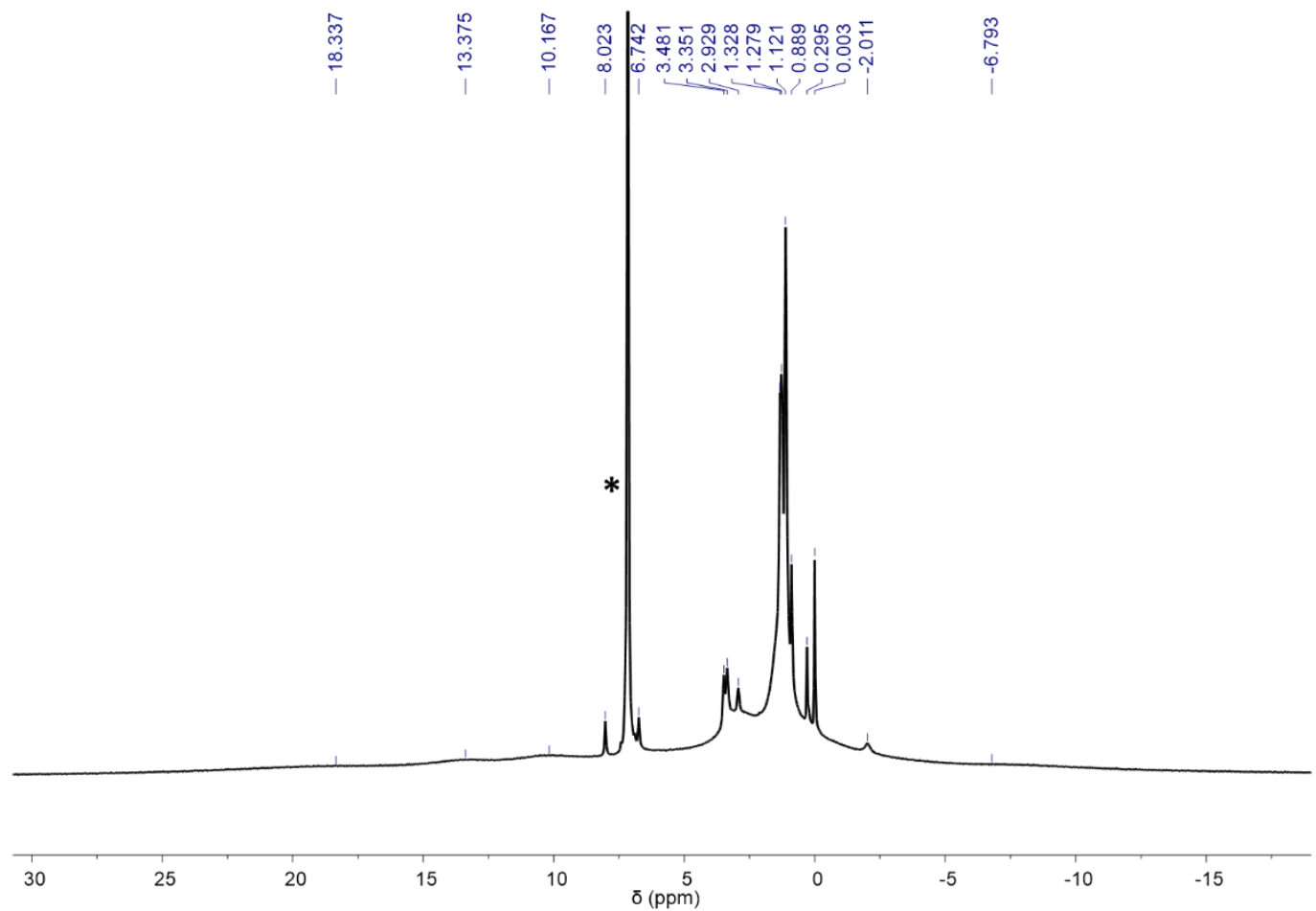

Figure S2 ${ }^{1} \mathrm{H}$ NMR spectrum (300 MHz, $\left.\mathrm{C}_{6} \mathrm{D}_{6}\right)$ of complex 2 (ca. $\left.0.1 \mathrm{M}\right)$. The asterisk denotes the protio-residual resonance of $\mathrm{C}_{6} \mathrm{D}_{5} \mathrm{H}$. 
FTIR spectra of the complexes

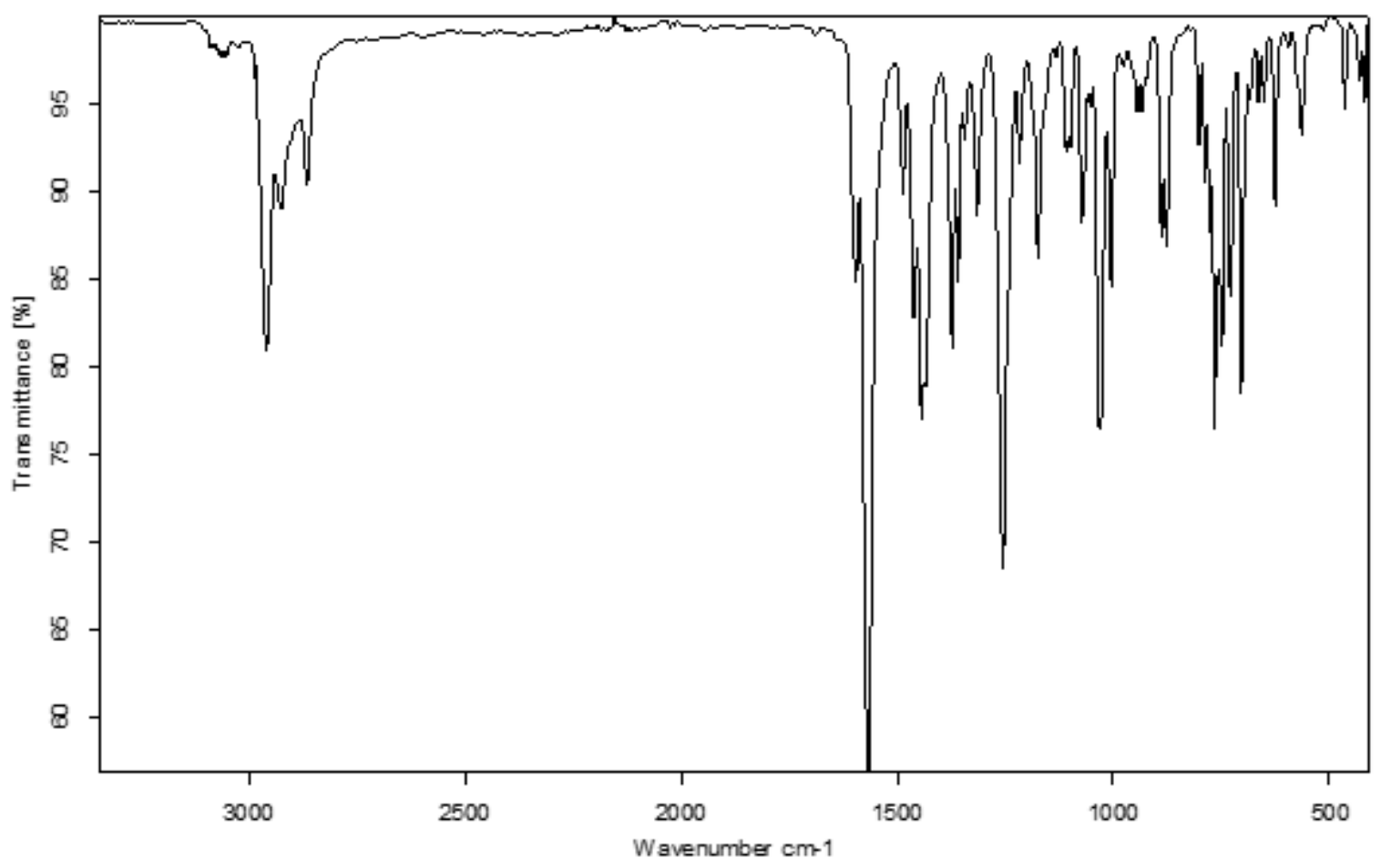

Figure S3 ATR-FTIR spectrum of complex 1.

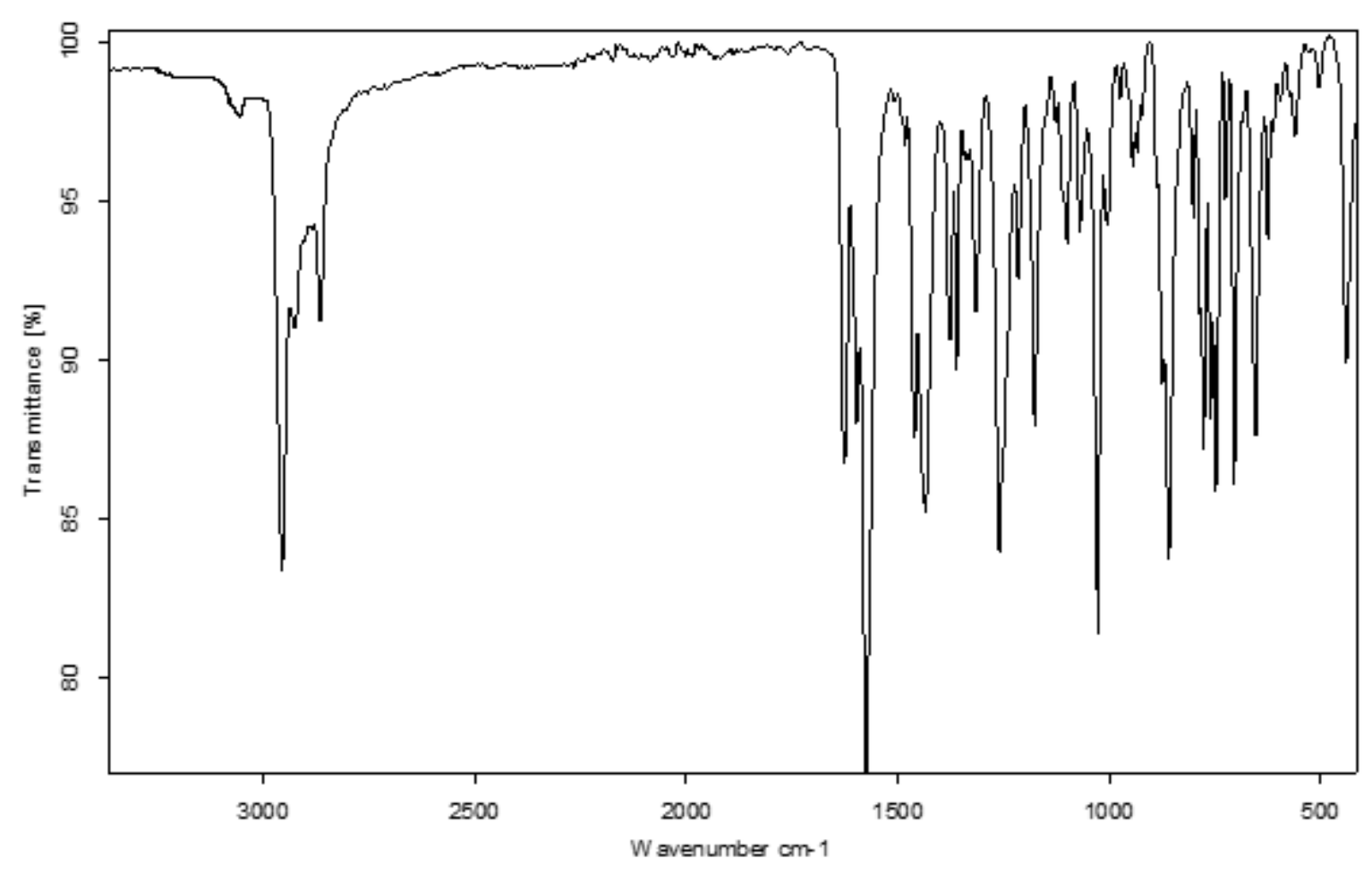

Figure S4 ATR-FTIR spectrum of complex 2. 


\section{Crystallographic data for complexes 1 and 2}

Table S1 Selected bond distances $(\AA)$ and bond angles $\left(^{\circ}\right)$ for complex 1 . The \# superscript corresponds to the bridging chloride atom or the $\mathrm{Mn} 1$ atom from the other half of the asymmetric unit.

\begin{tabular}{|c|c|c|c|}
\hline Bond lengths & & Bond angles & \\
\hline $\mathrm{Mn} 1-\mathrm{N} 1$ & $2.175(2)$ & $\mathrm{N} 1-\mathrm{Mn} 1-\mathrm{N} 2$ & $80.39(8)$ \\
\hline $\mathrm{Mn} 1-\mathrm{N} 2$ & $2.187(2)$ & N1-Mn1-N3 & $95.71(8)$ \\
\hline $\mathrm{Mn} 1-\mathrm{N} 3$ & $2.256(2)$ & $\mathrm{N} 2-\mathrm{Mn} 1-\mathrm{N} 3$ & $101.70(8)$ \\
\hline $\mathrm{Mn} 1-\mathrm{Cl1}^{\#}$ & $2.457(2)$ & $\mathrm{N} 1-\mathrm{Mn} 1-\mathrm{Cl1}^{\#}$ & $94.30(6)$ \\
\hline Mn1-C11 & $2.499(2)$ & $\mathrm{N} 2-\mathrm{Mn} 1-\mathrm{Cl1}^{\#}$ & $112.31(6)$ \\
\hline $\mathrm{N} 1-\mathrm{C} 5$ & $1.359(3)$ & $\mathrm{N} 3-\mathrm{Mn} 1-\mathrm{Cl1}^{\#}$ & $145.69(7)$ \\
\hline $\mathrm{N} 1-\mathrm{C} 2$ & $1.391(3)$ & $\mathrm{N} 1-\mathrm{Mn} 1-\mathrm{Cl1}$ & $174.63(6)$ \\
\hline $\mathrm{N} 2-\mathrm{C} 6$ & $1.304(3)$ & $\mathrm{N} 2-\mathrm{Mn} 1-\mathrm{Cl1}$ & $95.01(6)$ \\
\hline $\mathrm{N} 2-\mathrm{C} 21$ & $1.444(3)$ & N3-Mn1-C11 & $87.92(6)$ \\
\hline $\mathrm{C} 1-\mathrm{C} 5$ & $1.508(4)$ & 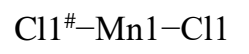 & $84.84(3)$ \\
\hline $\mathrm{C} 2-\mathrm{C} 3$ & $1.401(3)$ & $\mathrm{Mn} 1^{\#-C 11-M n 1}$ & $95.16(3)$ \\
\hline $\mathrm{C} 2-\mathrm{C} 6$ & $1.420(3)$ & $\mathrm{C} 5-\mathrm{N} 1-\mathrm{C} 2$ & $105.8(2)$ \\
\hline $\mathrm{C} 3-\mathrm{C} 4$ & $1.384(4)$ & $\mathrm{C} 6-\mathrm{N} 2-\mathrm{C} 21$ & $118.2(2)$ \\
\hline \multirow[t]{9}{*}{$\mathrm{C} 4-\mathrm{C} 5$} & $1.403(4)$ & $\mathrm{N} 1-\mathrm{C} 2-\mathrm{C} 3$ & $110.4(2)$ \\
\hline & & $\mathrm{N} 1-\mathrm{C} 2-\mathrm{C} 6$ & $120.5(2)$ \\
\hline & & $\mathrm{C} 3-\mathrm{C} 2-\mathrm{C} 6$ & $129.1(2)$ \\
\hline & & $\mathrm{C} 4-\mathrm{C} 3-\mathrm{C} 2$ & $106.0(2)$ \\
\hline & & $\mathrm{C} 3-\mathrm{C} 4-\mathrm{C} 5$ & $107.6(2)$ \\
\hline & & $\mathrm{N} 1-\mathrm{C} 5-\mathrm{C} 4$ & $110.2(2)$ \\
\hline & & $\mathrm{N} 1-\mathrm{C} 5-\mathrm{C} 1$ & $124.7(2)$ \\
\hline & & $\mathrm{C} 4-\mathrm{C} 5-\mathrm{C} 1$ & $125.0(2)$ \\
\hline & & $\mathrm{N} 2-\mathrm{C} 6-\mathrm{C} 2$ & $121.8(2)$ \\
\hline
\end{tabular}


Table S2 Selected bond distances $(\AA)$ and bond angles $\left(^{\circ}\right)$ for complex 2 .

\begin{tabular}{llll}
\hline Bond lengths & & \multicolumn{2}{l}{ Bond angles } \\
\hline Mn1-C33 & $2.107(2)$ & C33-Mn1-N1 & $128.36(8)$ \\
Mn1-N1 & $2.108(2)$ & C33-Mn1-N3 & $112.78(8)$ \\
Mn1-N3 & $2.198(2)$ & N1-Mn1-N3 & $105.87(6)$ \\
Mn1-N2 & $2.198(2)$ & C33-Mn1-N2 & $127.21(8)$ \\
Si1-C33 & $1.834(2)$ & N1-Mn1-N2 & $80.19(6)$ \\
Si1-C34 & $1.875(3)$ & N3-Mn1-N2 & $94.75(6)$ \\
N1-C5 & $1.357(2)$ & C33-Si1-C35 & $113.17(12)$ \\
N1-C2 & $1.385(2)$ & C33-Si1-C34 & $110.34(12)$ \\
N2-C6 & $1.296(2)$ & C33-Si1-C36 & $111.57(11)$ \\
N2-C21 & $1.432(2)$ & C5-N1-C2 & $106.27(14)$ \\
C1-C5 & $1.501(3)$ & C6-N2-C21 & $120.50(15)$ \\
C2-C3 & $1.393(3)$ & N1-C2-C3 & $110.06(17)$ \\
C2-C6 & $1.421(3)$ & N1-C2-C6 & $119.27(16)$ \\
C3-C4 & $1.394(3)$ & C3-C2-C6 & $130.65(17)$ \\
C4-C5 & $1.398(3)$ & C4-C3-C2 & $106.38(17)$ \\
& & C3-C4-C5 & $106.89(17)$ \\
& & N1-C5-C4 & $110.41(16)$ \\
& & N1-C5-C1 & $121.15(15)$ \\
& & C4-C5-C1 & $128.42(17)$ \\
& & N2-C6-C2 & $121.32(17)$ \\
\hline
\end{tabular}


Table S3 Crystallographic data for complexes $\mathbf{1}$ and $\mathbf{2 .}$

\begin{tabular}{lll}
\hline & \multicolumn{1}{c}{$\mathbf{1}$} & \multicolumn{1}{c}{$\mathbf{2}$} \\
\hline Formula & $\mathrm{C}_{37} \mathrm{H}_{48} \mathrm{ClMnN}_{3}$ & $\mathrm{C}_{41} \mathrm{H}_{59} \mathrm{MnN}_{3} \mathrm{Si}$ \\
$\mathrm{M}$ & 625.17 & 676.94 \\
$\lambda(\AA)$ & 0.71073 & 0.71073 \\
$\mathrm{~T}(\mathrm{~K})$ & 150 & 150 \\
Crystal system & Monoclinic & Monoclinic \\
Space group & $\mathrm{P} 2{ }_{1} / \mathrm{c}$ & $\mathrm{P} 2{ }_{1} / \mathrm{c}$ \\
$\mathrm{a}(\AA)$ & $13.166(3)$ & $10.5776(8)$ \\
$\mathrm{b}(\AA)$ & $31.108(8)$ & $18.7778(15)$ \\
$\mathrm{c}(\AA)$ & $9.748(2)$ & $19.8900(14)$ \\
$\alpha(\AA)$ & 90 & 90 \\
$\beta(\AA)$ & $94.802(8)$ & $93.980(3)$ \\
$\gamma(\AA)$ & 90 & 90 \\
V $\left(\AA^{3}\right)$ & $3978.2(2)$ & $3941.1(5)$ \\
$\mathrm{Z}$ & 4 & 4 \\
$\rho_{\text {calc }}\left(\mathrm{g} . \mathrm{cm}^{-3}\right)$ & 1.044 & 1.141 \\
$\mu\left(\mathrm{mm}{ }^{-1}\right)$ & 0.424 & 0.396 \\
Crystal size $(\mathrm{mm})$ & $0.05 \times 0.05 \times 0.02$ & $0.25 \times 0.20 \times 0.20$ \\
$\theta_{\text {max }}\left({ }^{\circ}\right)$ & 28.616 & 28.772 \\
Total data & 126177 & 36142 \\
Unique data & 9924 & 10231 \\
$\mathrm{R}_{\text {int }}$ & 0.1728 & 0.0521 \\
$\mathrm{R}[I>2 \sigma(I)]$ & 0.1290 & 0.0501 \\
$\mathrm{R}_{\mathrm{w}}$ & 0.1447 & 0.0725 \\
Goodness of fit & 1.098 & 1.002 \\
$\rho_{\text {min }}$ & -0.410 & -0.287 \\
$\rho_{\text {max }}$ & 0.372 & \\
\hline
\end{tabular}




\section{Additional data for the catalytic hydrosilylations}

Table S4 Hydrosilylation of aldehydes and ketones with $\mathrm{PhSiH}_{3}$ catalyzed by complex $\mathbf{2}$ - silane selectivity. The presented data correspond to full conversion of the substrates.

\begin{tabular}{|c|c|c|c|c|c|}
\hline Substrate & Time (h) & Product ratio $(\mathbf{A}: \mathbf{B})^{a}$ & Substrate & Time (h) & Product ratio $(\mathbf{A}: \mathbf{B})^{a}$ \\
\hline & 1 & $>99: 1.0$ & & 1 & $1.5: 1.0$ \\
\hline & 1 & $>99: 1.0$ & & 1 & $1.6: 1.0$ \\
\hline & 1 & $>99: 1.0$ & & 1 & $1.4: 1.0$ \\
\hline & 1 & $>99: 1.0$ & & 1 & 2.6:1.0 \\
\hline & 1 & $0.13: 1.0$ & & 48 & $0.14: 1.0$ \\
\hline & 1 & $>99: 1.0$ & & 4 & $<0.01: 1$ \\
\hline & 1 & $>99: 1.0$ & & 1 & $1.2: 1.0$ \\
\hline & 1 & $>99: 1.0$ & & 1 & $0.26: 1$ \\
\hline & 1 & $>99: 1.0$ & & & \\
\hline
\end{tabular}

Conditions: $1 \mathrm{~mol} \%$ of $2 ; 0.8 \mathrm{mmol}$ of carbonyl substrate; $0.8 \mathrm{mmol}$ of $\mathrm{PhSiH}_{3}$; Solvent: $\mathrm{C}_{6} \mathrm{D}_{6}(0.5 \mathrm{~mL}) ;$ Temperature: $25^{\circ} \mathrm{C}$.

${ }^{a}$ Determined by ${ }^{1} \mathrm{H}$ NMR spectroscopy. 


\section{NMR data for the conversion of the aldehydes and ketones to the corresponding silanes catalyzed by complex 2}

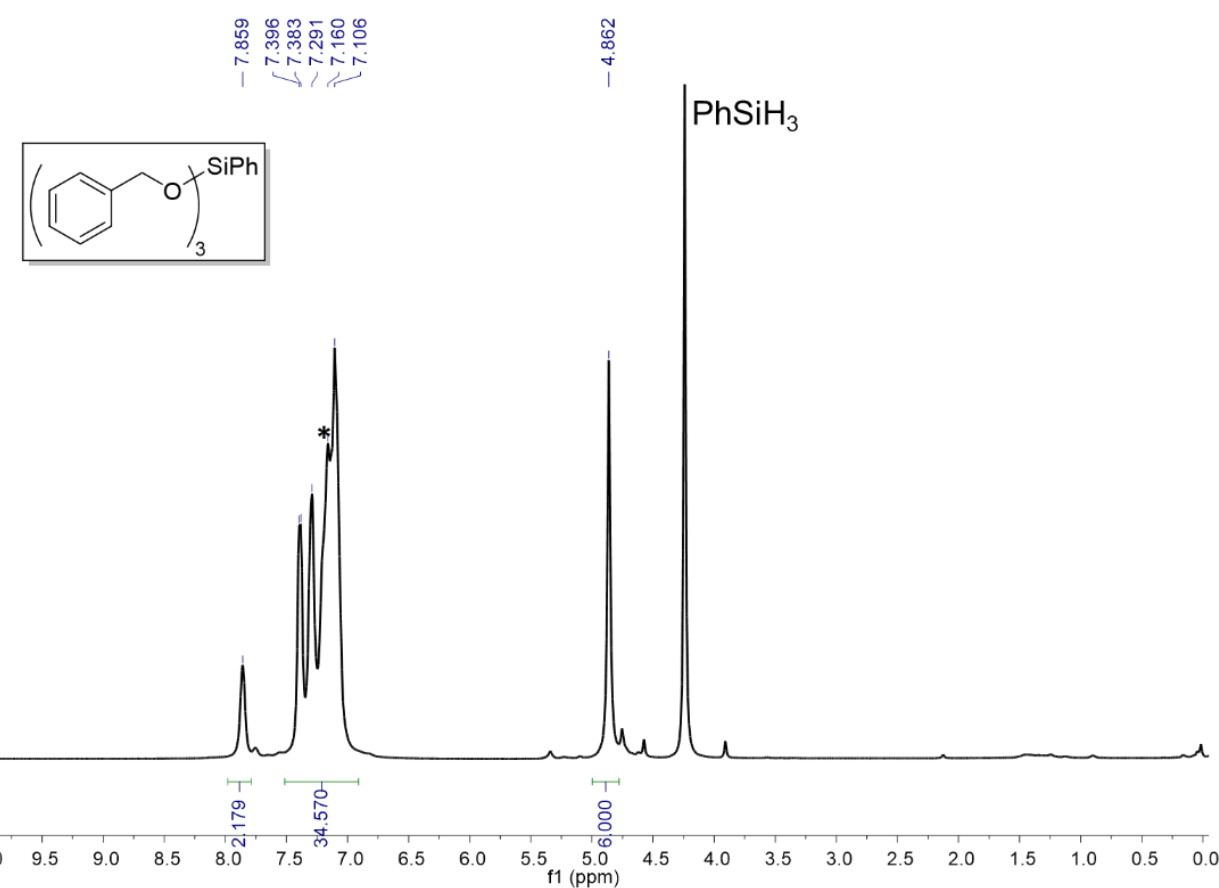

Figure S5 ${ }^{1} \mathrm{H}$ NMR spectrum $\left(300 \mathrm{MHz}, \mathrm{C}_{6} \mathrm{D}_{6}\right)$ of the catalytic hydrosilylation reaction of benzaldehyde with $\mathrm{PhSiH}_{3}$ catalyzed by complex 2 . The asterisk denotes the protio-residual resonance of $\mathrm{C}_{6} \mathrm{D}_{5} \mathrm{H}$ and all other unassigned peaks correspond to the silane $\mathrm{PhSi}\left(\mathrm{OCH}_{2} \mathrm{Ph}\right)_{3}$.

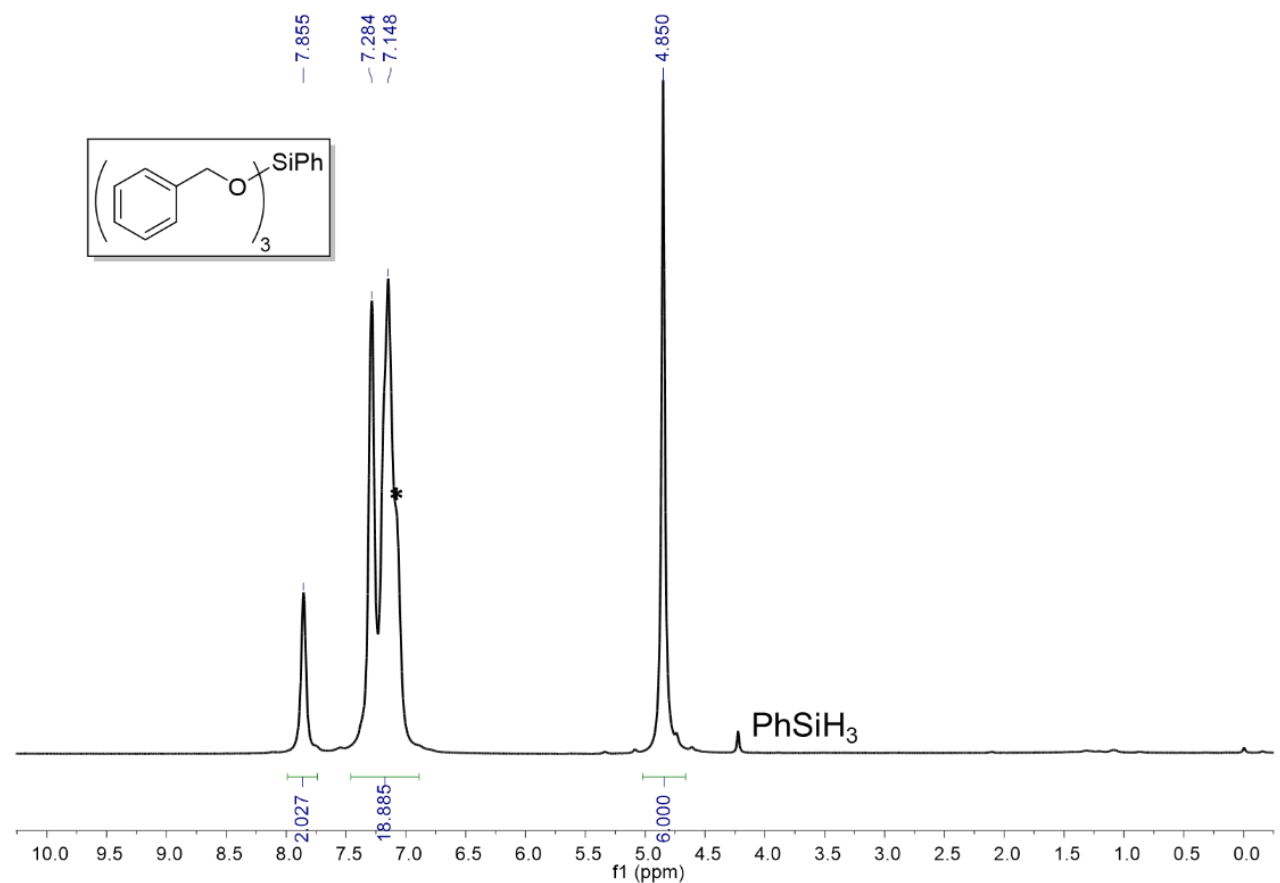

Figure S6 ${ }^{1} \mathrm{H}$ NMR spectrum $\left(300 \mathrm{MHz}, \mathrm{C}_{6} \mathrm{D}_{6}\right)$ of the catalytic hydrosilylation reaction of benzaldehyde with $1 / 3$ equivalents of $\mathrm{PhSiH}_{3}$ catalyzed by complex 2 . The asterisk denotes the protio-residual resonance of $\mathrm{C}_{6} \mathrm{D}_{5} \mathrm{H}$ and all other unassigned peaks correspond to the silane $\mathrm{PhSi}\left(\mathrm{OCH}_{2} \mathrm{Ph}\right)_{3}$. 


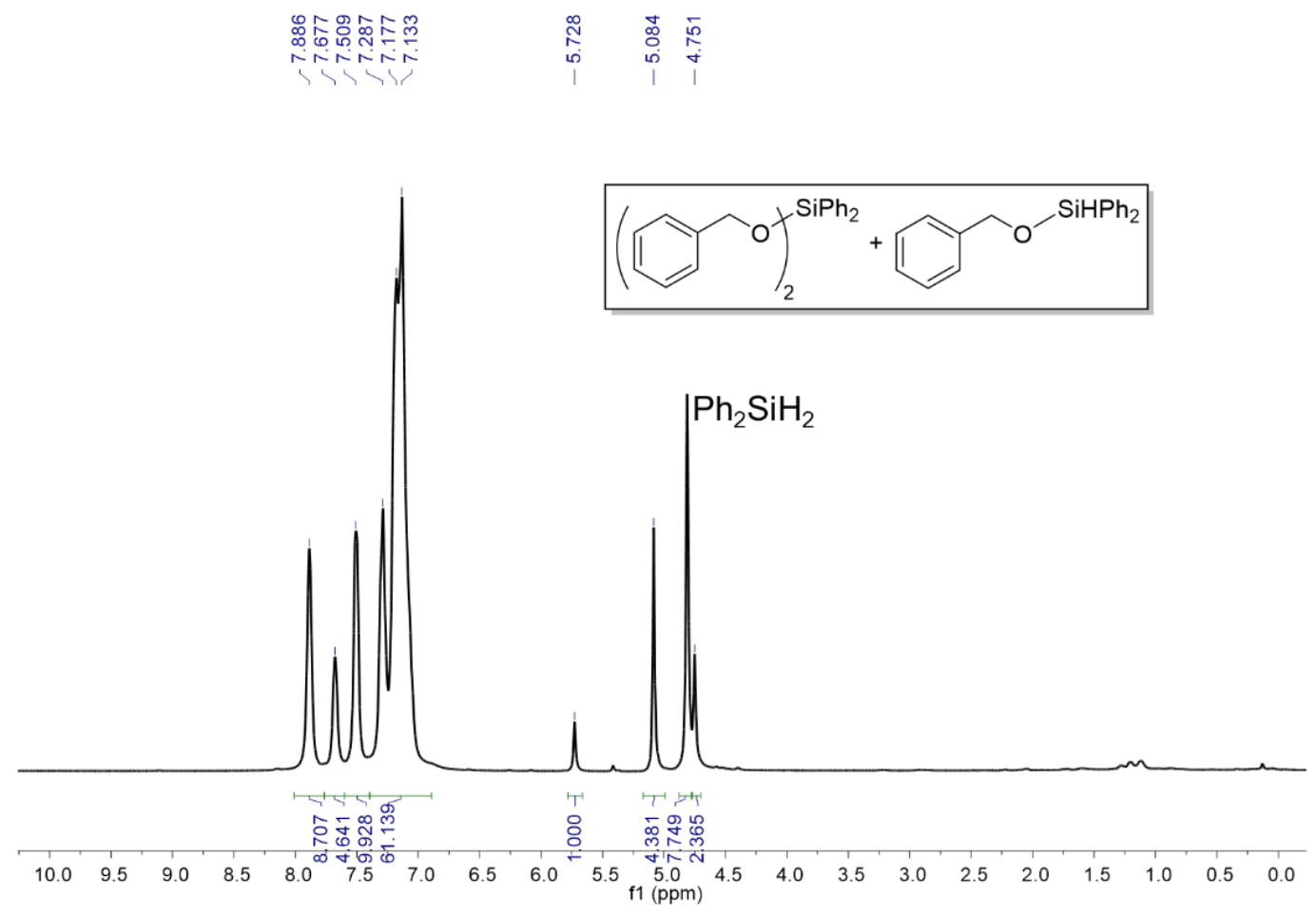

Figure $\mathbf{S} 7{ }^{1} \mathrm{H}$ NMR spectrum ( $300 \mathrm{MHz}, \mathrm{C}_{6} \mathrm{D}_{6}$ ) of the catalytic hydrosilylation reaction of benzaldehyde with $\mathrm{Ph}_{2} \mathrm{SiH}_{2}$ catalyzed by complex 2 . The protio-residual resonance of $\mathrm{C}_{6} \mathrm{D}_{5} \mathrm{H}$ has been overlapped by the aromatic resonances and all other unassigned peaks correspond to the mixture of silanes $\mathrm{Ph}_{2} \mathrm{SiH}\left(\mathrm{OCH}_{2} \mathrm{Ph}\right)$ and $\mathrm{Ph}_{2} \mathrm{Si}\left(\mathrm{OCH}_{2} \mathrm{Ph}\right)$.

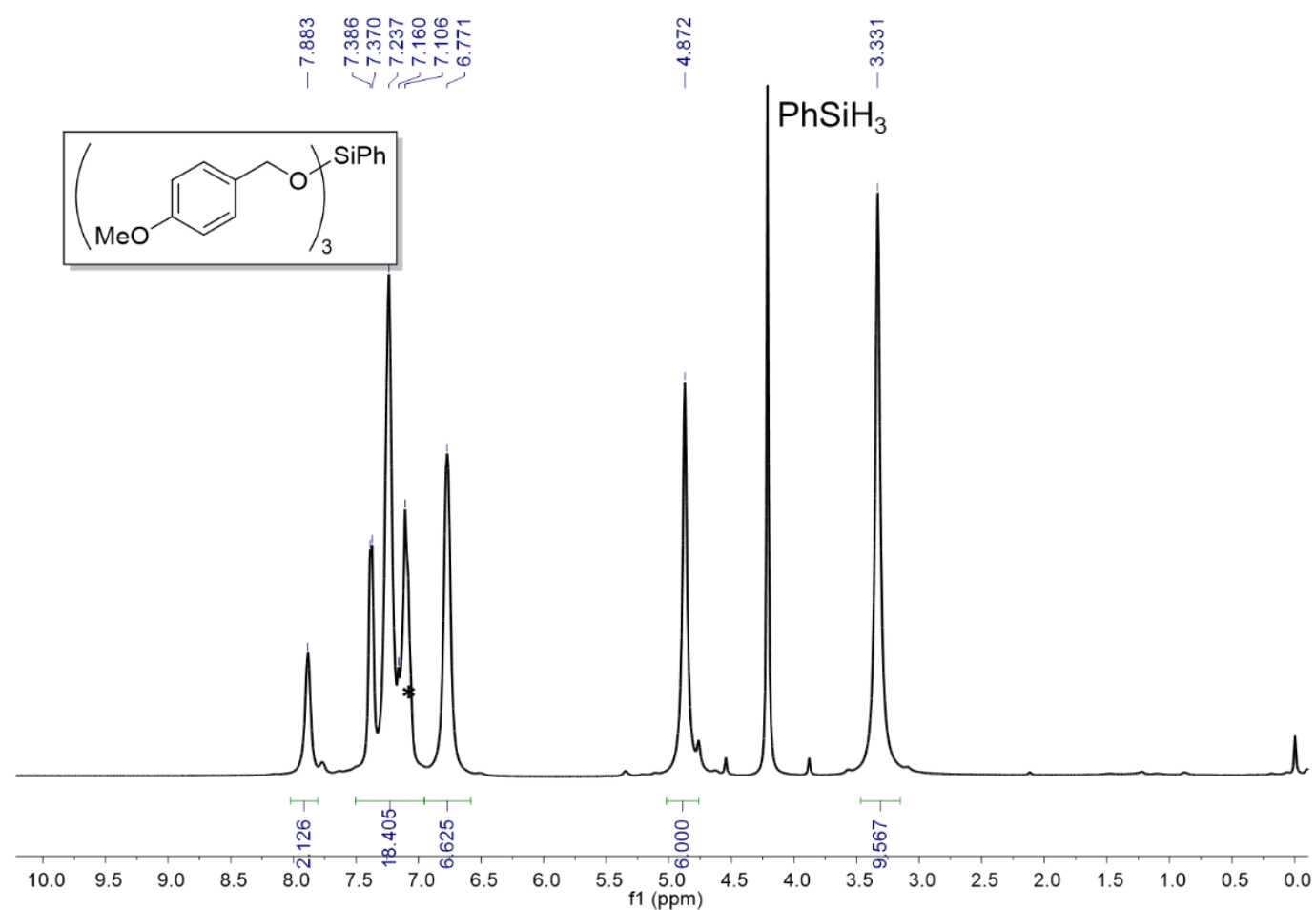

Figure S8 ${ }^{1} \mathrm{H}$ NMR spectrum $\left(300 \mathrm{MHz}, \mathrm{C}_{6} \mathrm{D}_{6}\right)$ of the catalytic hydrosilylation reaction of 4-methoxybenzaldehyde with $\mathrm{PhSiH}_{3}$ catalyzed by complex 2 . The asterisk denotes the protio-residual resonance of $\mathrm{C}_{6} \mathrm{D}_{5} \mathrm{H}$ and all other unassigned peaks correspond to the silane $\mathrm{PhSi}_{2}\left[\mathrm{OCH}_{2}\left(4-\mathrm{OMeC}_{6} \mathrm{H}_{4}\right)\right]_{3}$. 


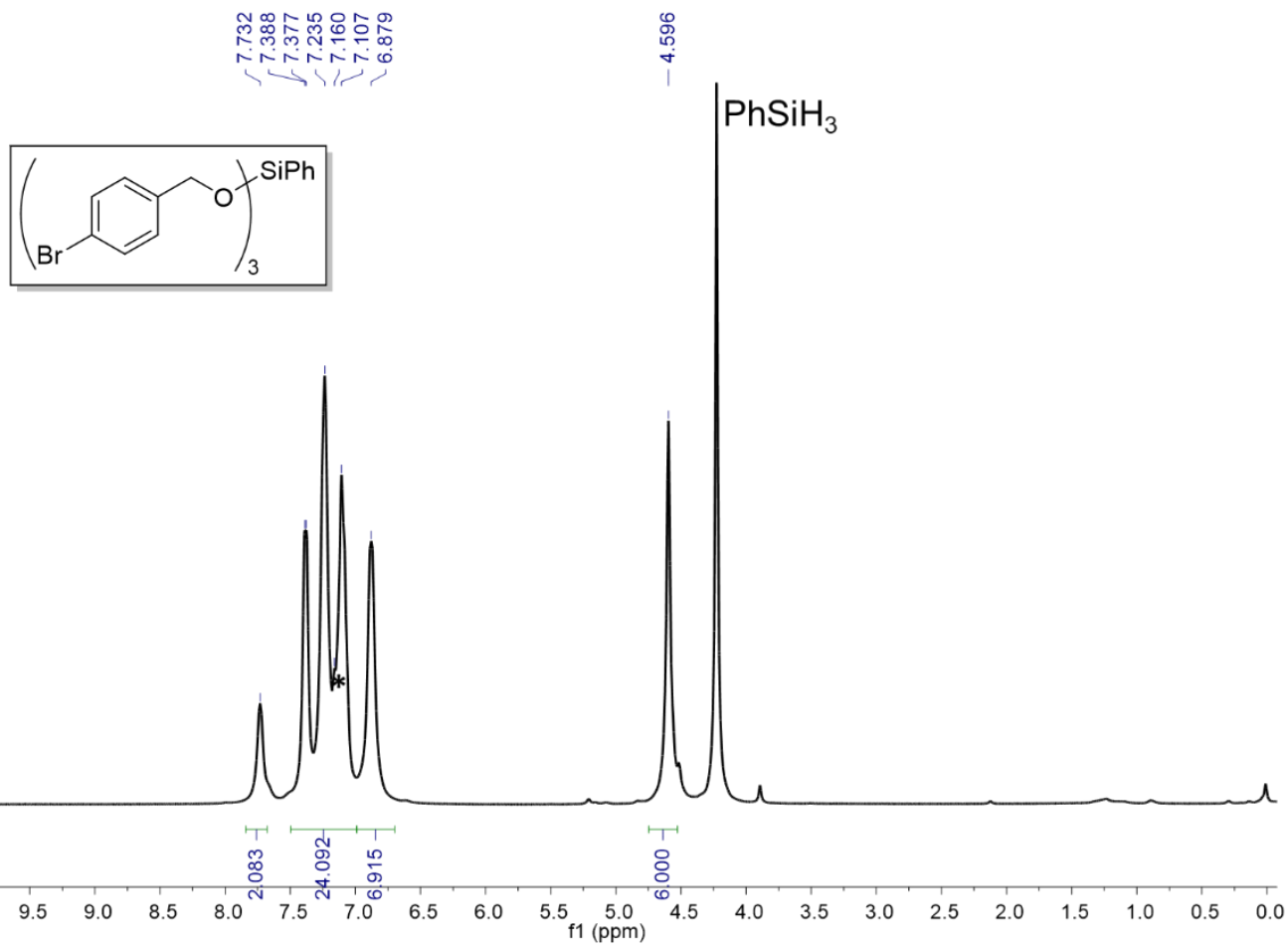

Figure S9 ${ }^{1} \mathrm{H}$ NMR spectrum $\left(300 \mathrm{MHz}, \mathrm{C}_{6} \mathrm{D}_{6}\right)$ of the catalytic hydrosilylation reaction of 4-bromobenzaldehyde with $\mathrm{PhSiH}_{3}$ catalyzed by complex 2 . The asterisk denotes the protio-residual resonance of $\mathrm{C}_{6} \mathrm{D}_{5} \mathrm{H}$ and all other unassigned peaks correspond to the silane $\mathrm{PhSi}\left[\mathrm{OCH}_{2}\left(4-\mathrm{BrC}_{6} \mathrm{H}_{4}\right)\right]_{3}$.
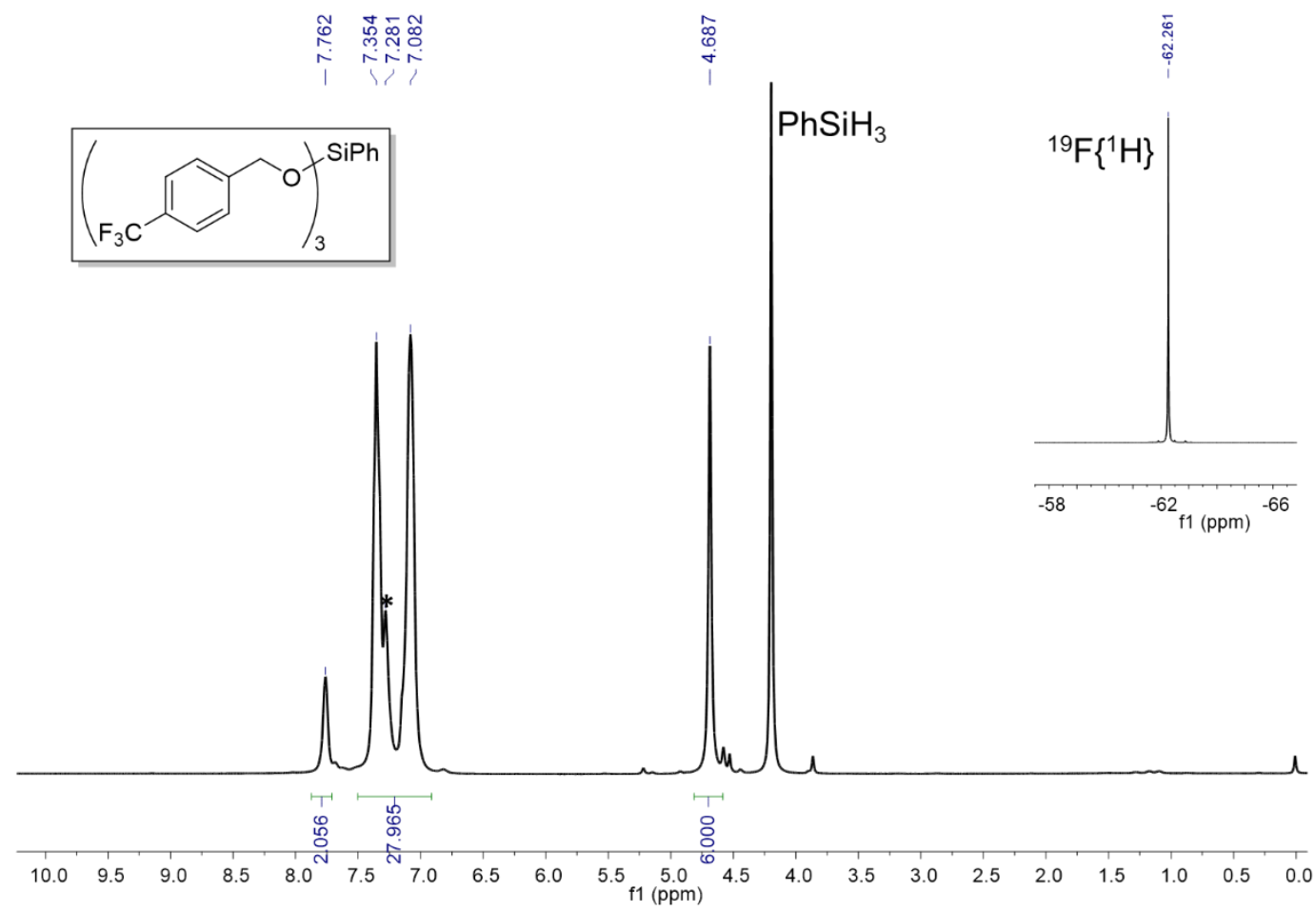

Figure S10 ${ }^{1} \mathrm{H}$ NMR spectrum (300 MHz, $\mathrm{C}_{6} \mathrm{D}_{6}$ ) of the catalytic hydrosilylation reaction of 4-(trifluoromethyl)benzaldehyde with $\mathrm{PhSiH}_{3}$ catalyzed by complex 2 . The asterisk denotes the protio-residual resonance of $\mathrm{C}_{6} \mathrm{D}_{5} \mathrm{H}$ and all other unassigned peaks correspond to the silane $\mathrm{PhSi}\left[\mathrm{OCH}_{2}\left(4-\left(\mathrm{CF}_{3}\right) \mathrm{C}_{6} \mathrm{H}_{4}\right)\right] 3$. The inset shows the respective ${ }^{19} \mathrm{~F}\left\{{ }^{1} \mathrm{H}\right\}$ spectrum. 


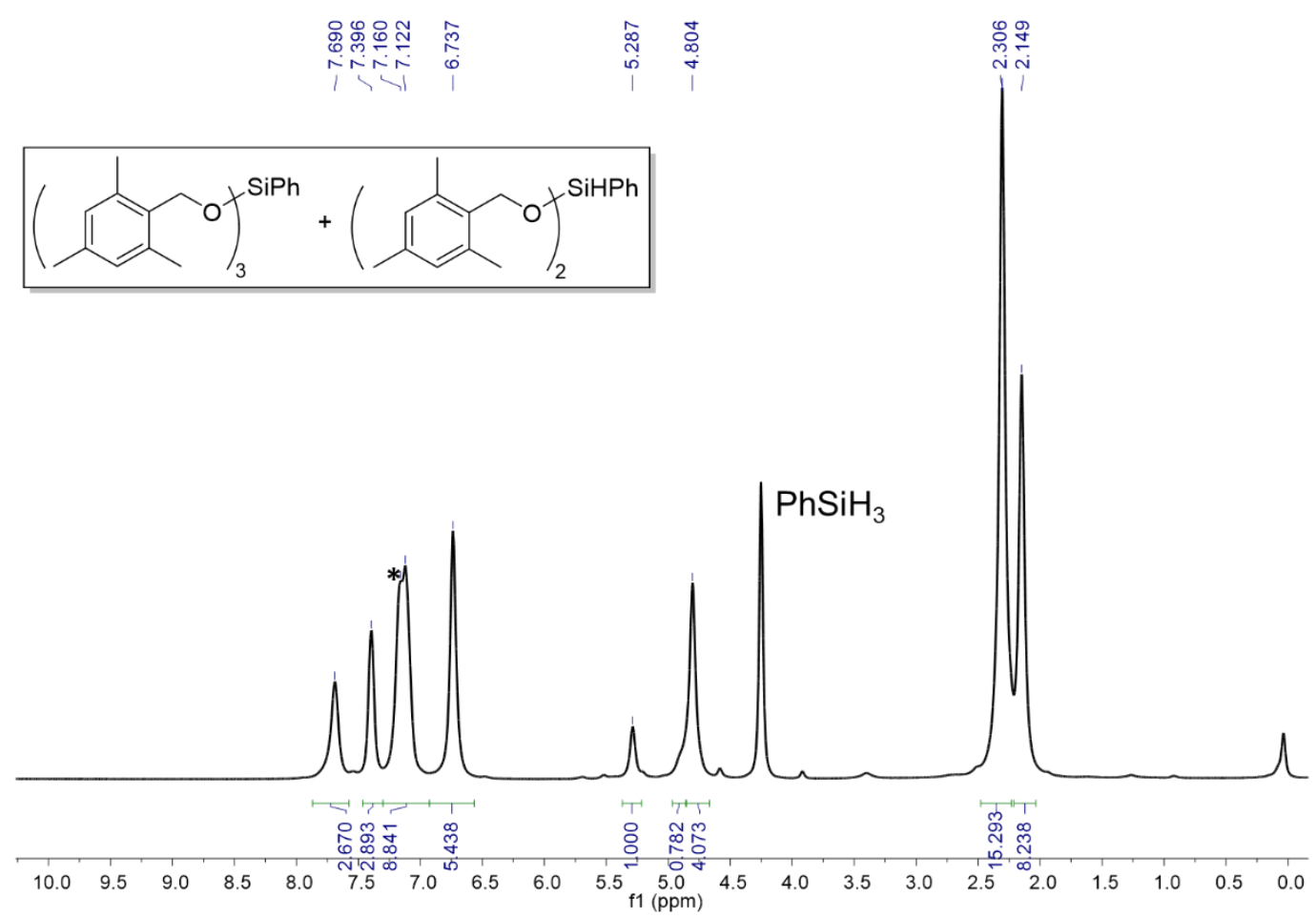

Figure S11 ${ }^{1} \mathrm{H}$ NMR spectrum (300 MHz, $\mathrm{C}_{6} \mathrm{D}_{6}$ ) of the catalytic hydrosilylation reaction of 2,4,6-trimethylbenzaldehyde with $\mathrm{PhSiH}_{3}$ catalyzed by complex 2 . The asterisk denotes the protio-residual resonance of $\mathrm{C}_{6} \mathrm{D}_{5} \mathrm{H}$ and all other unassigned peaks correspond to the mixture of silanes $\mathrm{PhSi}\left[\mathrm{OCH}_{2}\left(2,4,6-\left(\mathrm{CH}_{3}\right)_{3} \mathrm{C}_{6} \mathrm{H}_{2}\right)\right]_{3}$ and $\mathrm{PhHSi}_{2}\left[\mathrm{OCH}_{2}\left(2,4,6-\left(\mathrm{CH}_{3}\right)_{3} \mathrm{C}_{6} \mathrm{H}_{2}\right)\right]_{2}$.

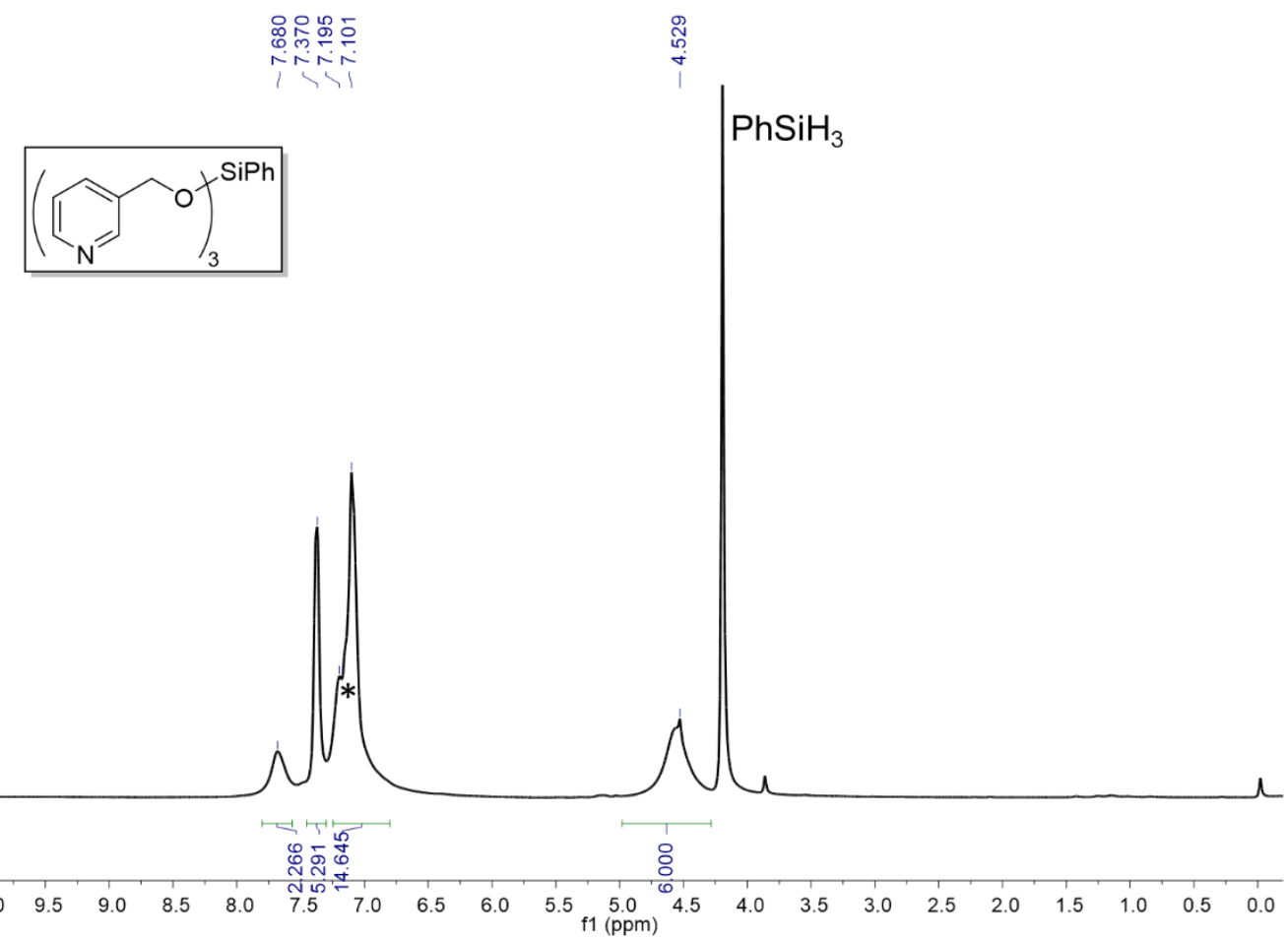

Figure S12 ${ }^{1} \mathrm{H}$ NMR spectrum (300 MHz, $\left.\mathrm{C}_{6} \mathrm{D}_{6}\right)$ of the catalytic hydrosilylation reaction of 3-pyridinecarboxaldehyde with $\mathrm{PhSiH}_{3}$ catalyzed by complex 2 . The asterisk denotes the protio-residual resonance of $\mathrm{C}_{6} \mathrm{D}_{5} \mathrm{H}$ and all other unassigned peaks correspond to the silane $\mathrm{PhSi}\left[\mathrm{OCH}_{2}\left(\mathrm{C}_{5} \mathrm{H}_{4} \mathrm{~N}\right)\right]_{3}$. 


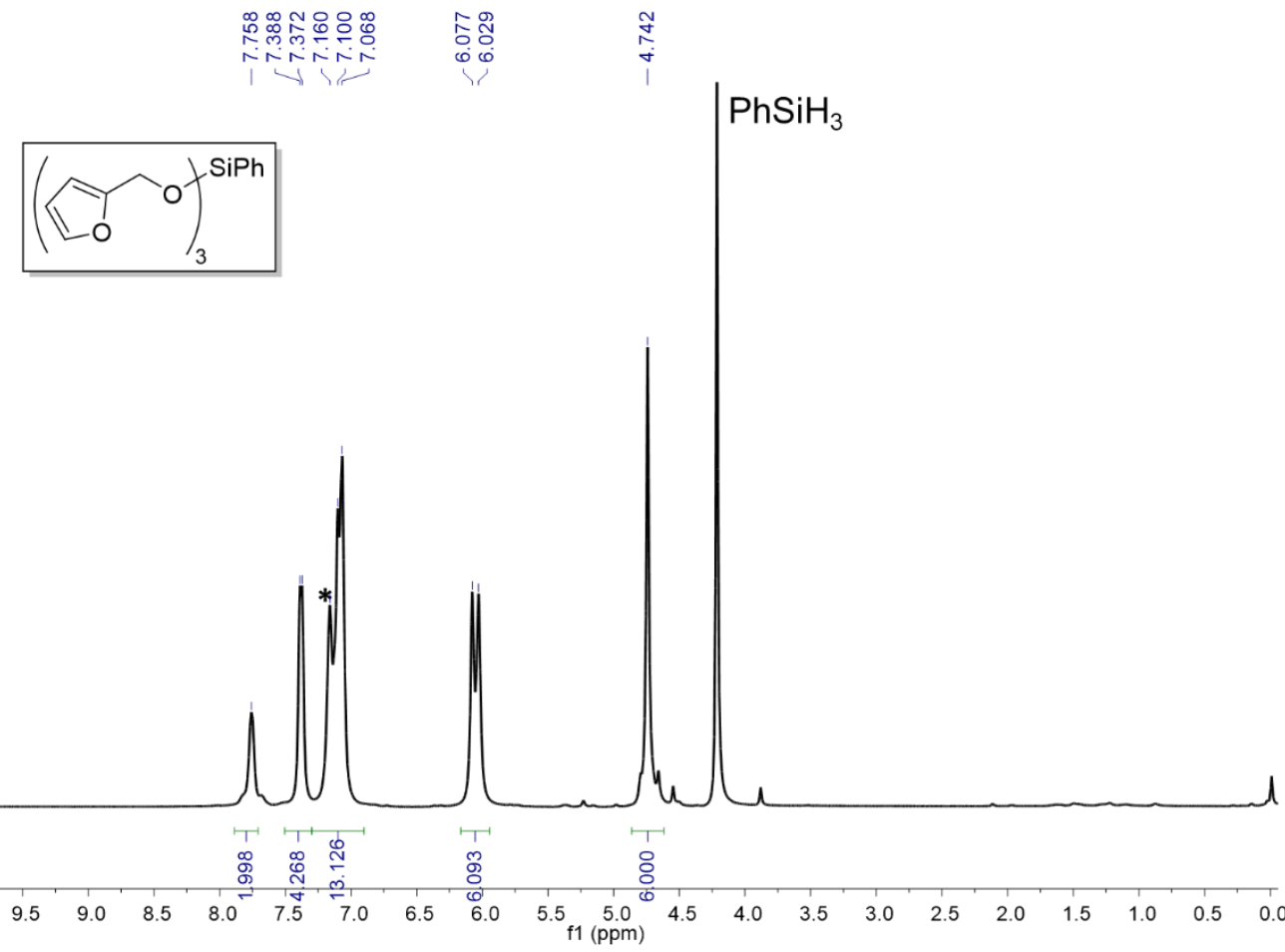

Figure $\mathrm{S13}{ }^{1} \mathrm{H}$ NMR spectrum $\left(300 \mathrm{MHz}, \mathrm{C}_{6} \mathrm{D}_{6}\right)$ of the catalytic hydrosilylation reaction of furfural with $\mathrm{PhSiH}_{3}$ catalyzed by complex 2 . The asterisk denotes the protio-residual resonance of $\mathrm{C}_{6} \mathrm{D}_{5} \mathrm{H}$ and all other unassigned peaks correspond to the silane $\mathrm{PhSi}\left[\mathrm{OCH}_{2}\left(\mathrm{C}_{4} \mathrm{H}_{3} \mathrm{O}\right)\right]_{3}$.

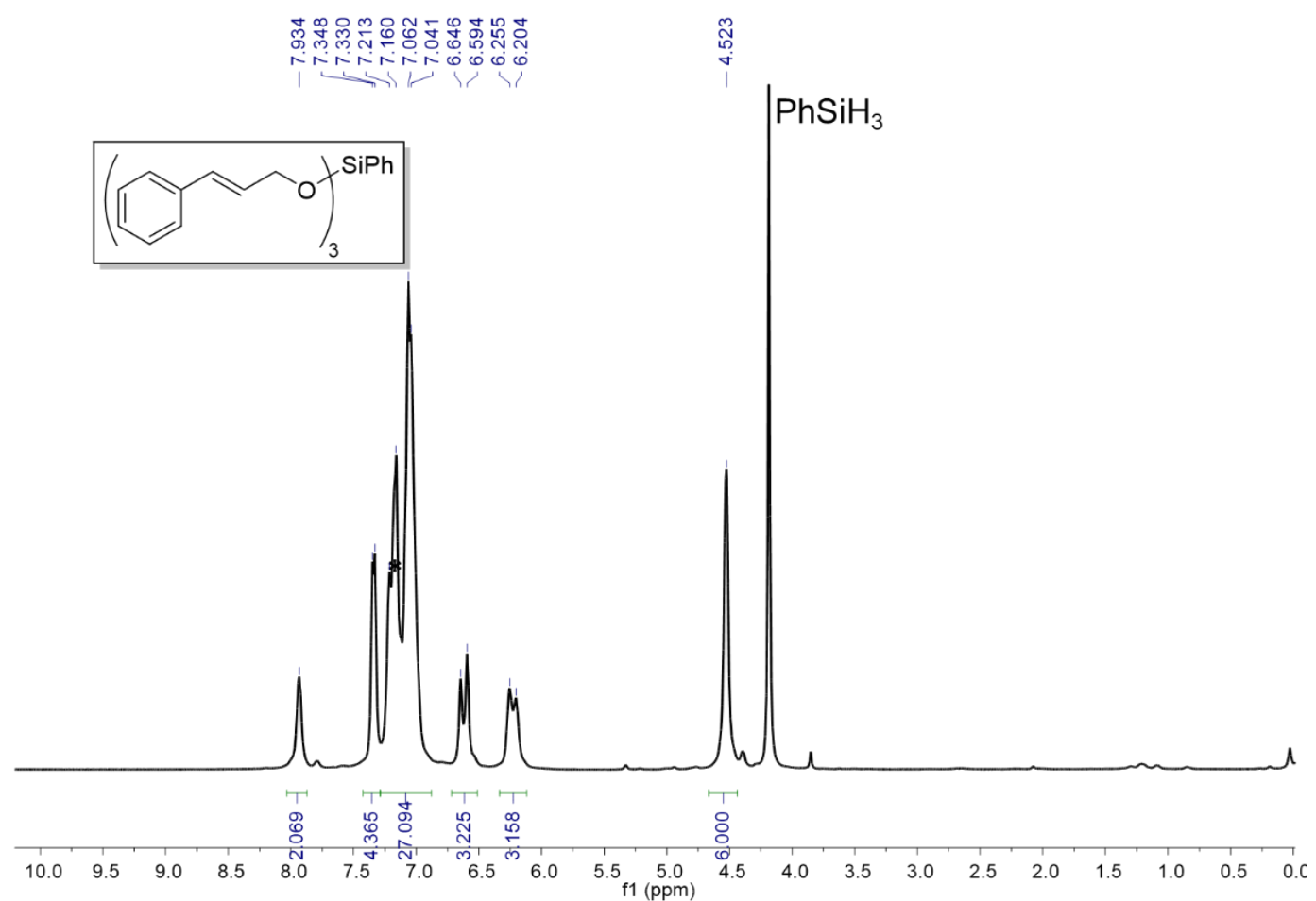

Figure S14 ${ }^{1} \mathrm{H}$ NMR spectrum $\left(300 \mathrm{MHz}, \mathrm{C}_{6} \mathrm{D}_{6}\right)$ of the catalytic hydrosilylation reaction of trans-cinnamaldehyde with $\mathrm{PhSiH}_{3}$ catalyzed by complex 2 . The asterisk denotes the protio-residual resonance of $\mathrm{C}_{6} \mathrm{D}_{5} \mathrm{H}$ and all other unassigned peaks correspond to the silane $\mathrm{PhSi}\left(\mathrm{OCH}_{2} \mathrm{CHCHPh}\right)_{3}$. 


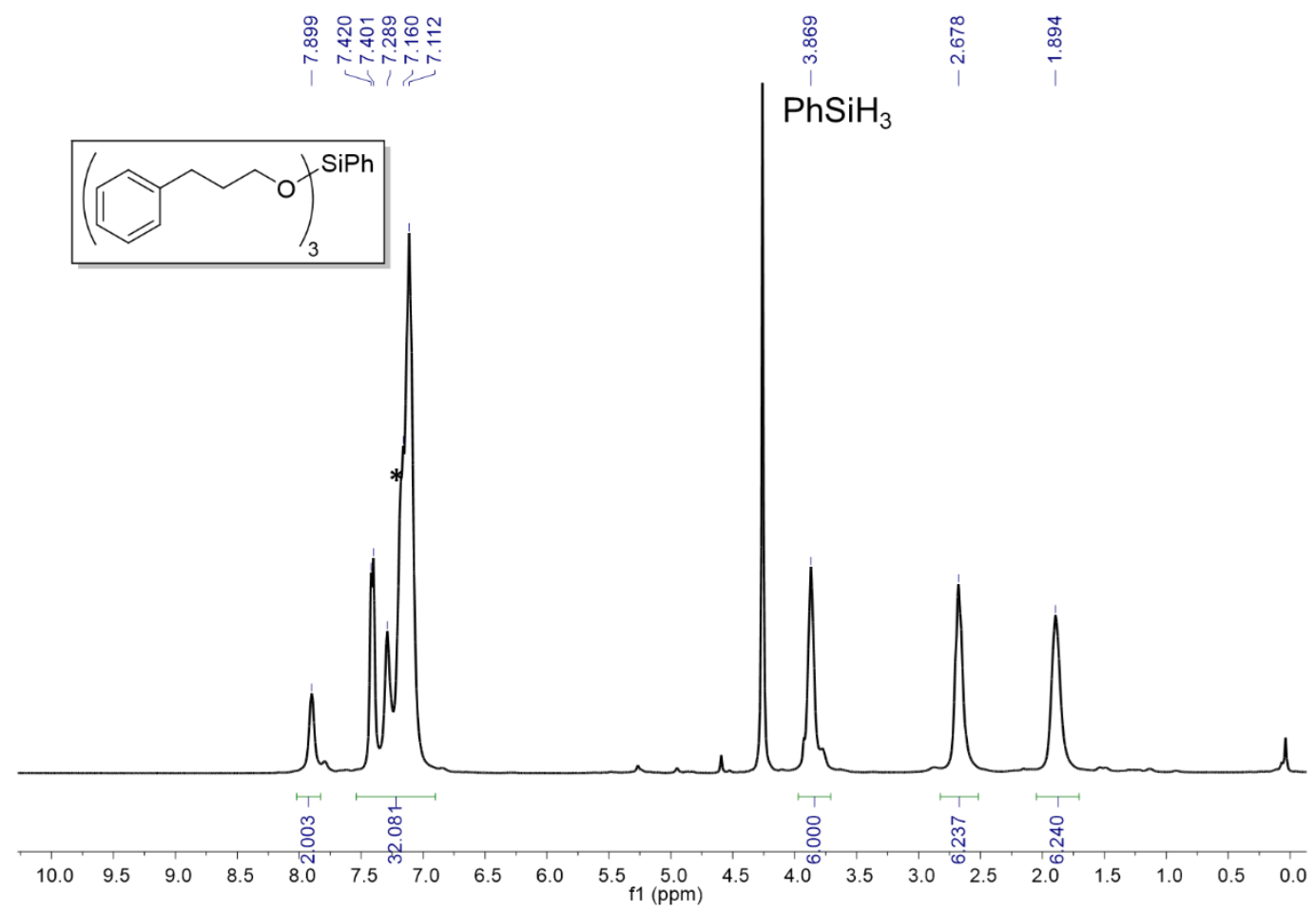

Figure S15 ${ }^{1} \mathrm{H}$ NMR spectrum $\left(300 \mathrm{MHz}, \mathrm{C}_{6} \mathrm{D}_{6}\right)$ of the catalytic hydrosilylation reaction of 3-phenylpropanal (hydrocinnamaldehyde) with $\mathrm{PhSiH}_{3}$ catalyzed by complex 2 . The asterisk denotes the protio-residual resonance of $\mathrm{C}_{6} \mathrm{D}_{5} \mathrm{H}$ and all other unassigned peaks correspond to the silane $\mathrm{PhSi}\left(\mathrm{OCH}_{2} \mathrm{CH}_{2} \mathrm{CH}_{2} \mathrm{Ph}\right)_{3}$.

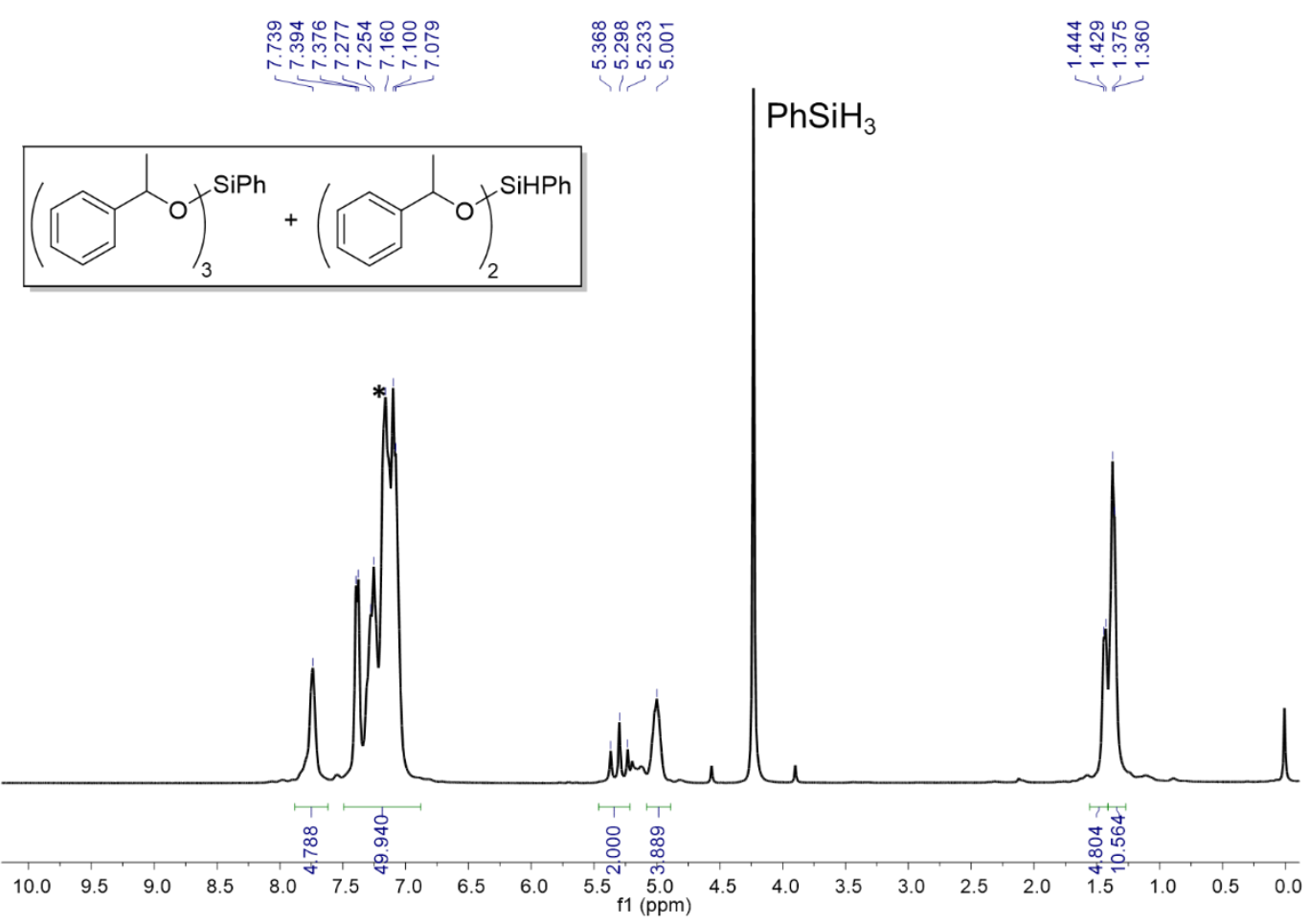

Figure $\mathrm{S16}^{1} \mathrm{H}$ NMR spectrum $\left(300 \mathrm{MHz}, \mathrm{C}_{6} \mathrm{D}_{6}\right)$ of the catalytic hydrosilylation reaction of acetophenone with $\mathrm{PhSiH}_{3}$ catalyzed by complex 2 . The asterisk denotes the protio-residual resonance of $\mathrm{C}_{6} \mathrm{D}_{5} \mathrm{H}$ and all other unassigned peaks correspond to the mixture of silanes $\mathrm{PhSi}\left[\mathrm{OCH}\left(\mathrm{CH}_{3}\right) \mathrm{Ph}\right]_{3}$ and $\mathrm{PhHSi}\left[\mathrm{OCH}\left(\mathrm{CH}_{3}\right) \mathrm{Ph}\right]_{2}$. 


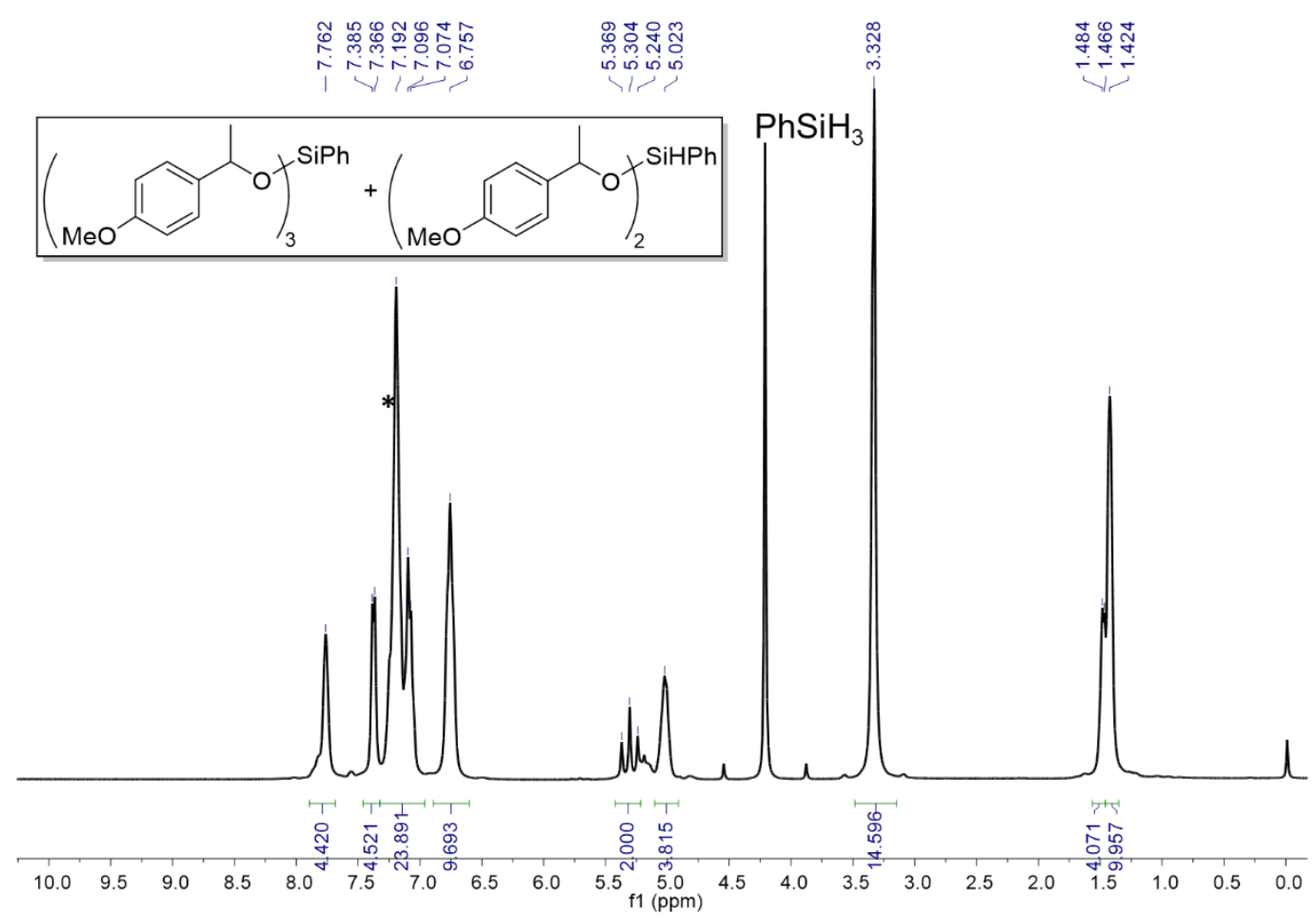

Figure $\mathbf{S 1 7}{ }^{1} \mathrm{H}$ NMR spectrum (300 MHz, $\mathrm{C}_{6} \mathrm{D}_{6}$ ) of the catalytic hydrosilylation reaction of 4'-methoxyacetophenone with $\mathrm{PhSiH}_{3}$ catalyzed by complex 2 . The asterisk denotes the protio-residual resonance of $\mathrm{C}_{6} \mathrm{D}_{5} \mathrm{H}$ and all other unassigned peaks correspond to the mixture of silanes $\mathrm{PhSi}\left[\mathrm{OCH}\left(\mathrm{CH}_{3}\right)\left(4-\mathrm{OMeC}_{6} \mathrm{H}_{4}\right)\right]_{3}$ and $\mathrm{PhHSi}\left[\mathrm{OCH}\left(\mathrm{CH}_{3}\right)\left(4-\mathrm{OMeC}_{6} \mathrm{H}_{4}\right)\right]_{2}$.

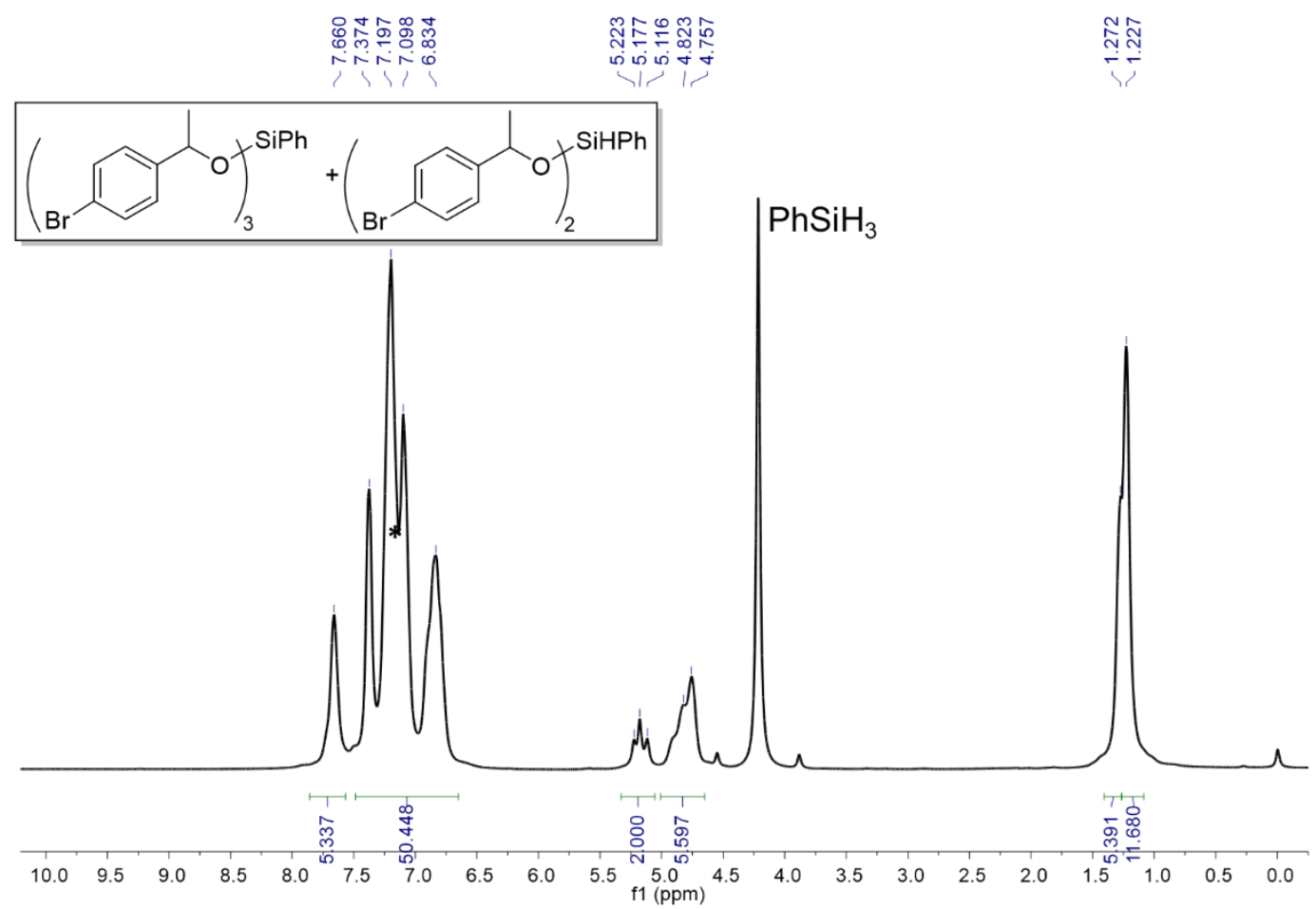

Figure S18 ${ }^{1} \mathrm{H}$ NMR spectrum (300 MHz, C6 $\mathrm{D}_{6}$ ) of the catalytic hydrosilylation reaction of 4'-bromoacetophenone with $\mathrm{PhSiH}_{3}$ catalyzed by complex 2 . The asterisk denotes the protio-residual resonance of $\mathrm{C}_{6} \mathrm{D}_{5} \mathrm{H}$ and all other unassigned peaks correspond to the mixture of silanes $\mathrm{PhSi}\left[\mathrm{OCH}\left(\mathrm{CH}_{3}\right)\left(4-\mathrm{BrC}_{6} \mathrm{H}_{4}\right)\right]_{3}$ and $\mathrm{PhHSi}\left[\mathrm{OCH}\left(\mathrm{CH}_{3}\right)\left(4-\mathrm{BrC}_{6} \mathrm{H}_{4}\right)\right]_{2}$. 


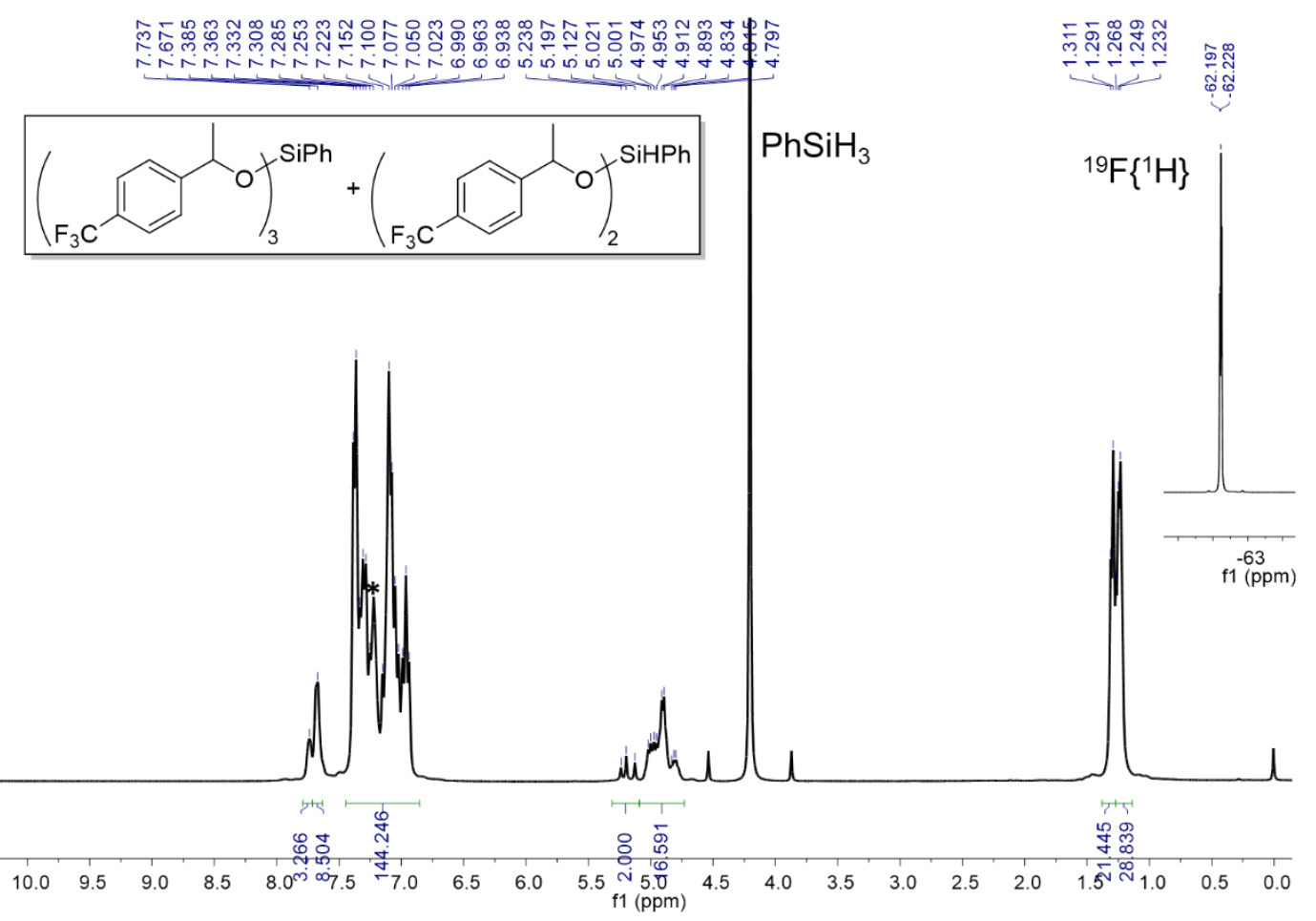

Figure S19 ${ }^{1} \mathrm{H}$ NMR spectrum $\left(300 \mathrm{MHz}, \mathrm{C}_{6} \mathrm{D}_{6}\right)$ of the catalytic hydrosilylation reaction of 4'-

(trifluoromethyl)acetophenone with $\mathrm{PhSiH}_{3}$ catalyzed by complex 2. The asterisk denotes the protio-residual resonance of

$\mathrm{C}_{6} \mathrm{D}_{5} \mathrm{H}$ and all other unassigned peaks correspond to the mixture of silanes $\mathrm{PhSi}\left\{\mathrm{OCH}\left(\mathrm{CH}_{3}\right)\left[4-\left(\mathrm{CF}_{3}\right) \mathrm{C}_{6} \mathrm{H}_{4}\right]\right\}_{3}$ and $\mathrm{PhHSi}\left\{\mathrm{OCH}\left(\mathrm{CH}_{3}\right)\left[4-\left(\mathrm{CF}_{3}\right) \mathrm{C}_{6} \mathrm{H}_{4}\right]\right\}_{2}$. The inset shows the respective ${ }^{19} \mathrm{~F}\left\{{ }^{1} \mathrm{H}\right\}$ spectrum.

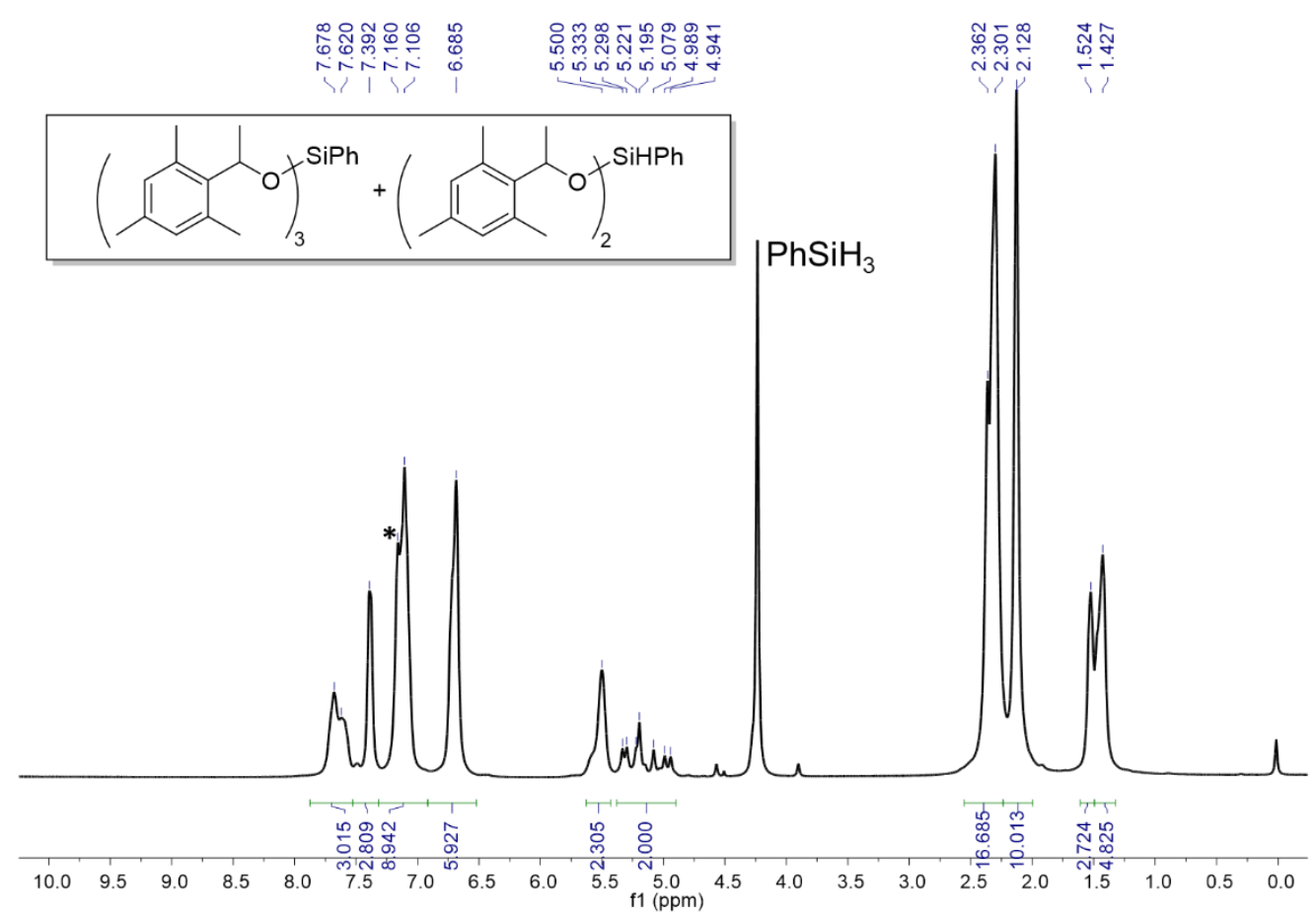

Figure S20 ${ }^{1} \mathrm{H}$ NMR spectrum (300 MHz, C6 $\mathrm{D}_{6}$ ) of the catalytic hydrosilylation reaction of 2',4',6'-trimethylacetophenone with $\mathrm{PhSiH}_{3}$ catalyzed by complex 2 . The asterisk denotes the protio-residual resonance of $\mathrm{C}_{6} \mathrm{D}_{5} \mathrm{H}$ and all other unassigned peaks correspond to the mixture of silanes $\mathrm{PhSi}\left\{\mathrm{OCH}\left(\mathrm{CH}_{3}\right)\left[2,4,6-\left(\mathrm{CH}_{3}\right)_{3} \mathrm{C}_{6} \mathrm{H}_{2}\right]\right\}_{3}$ and $\mathrm{PhHSi}\left\{\mathrm{OCH}\left(\mathrm{CH}_{3}\right)[2,4,6-\right.$

$\left.\left.\left(\mathrm{CH}_{3}\right)_{3} \mathrm{C}_{6} \mathrm{H}_{2}\right]\right\}_{2}$. 


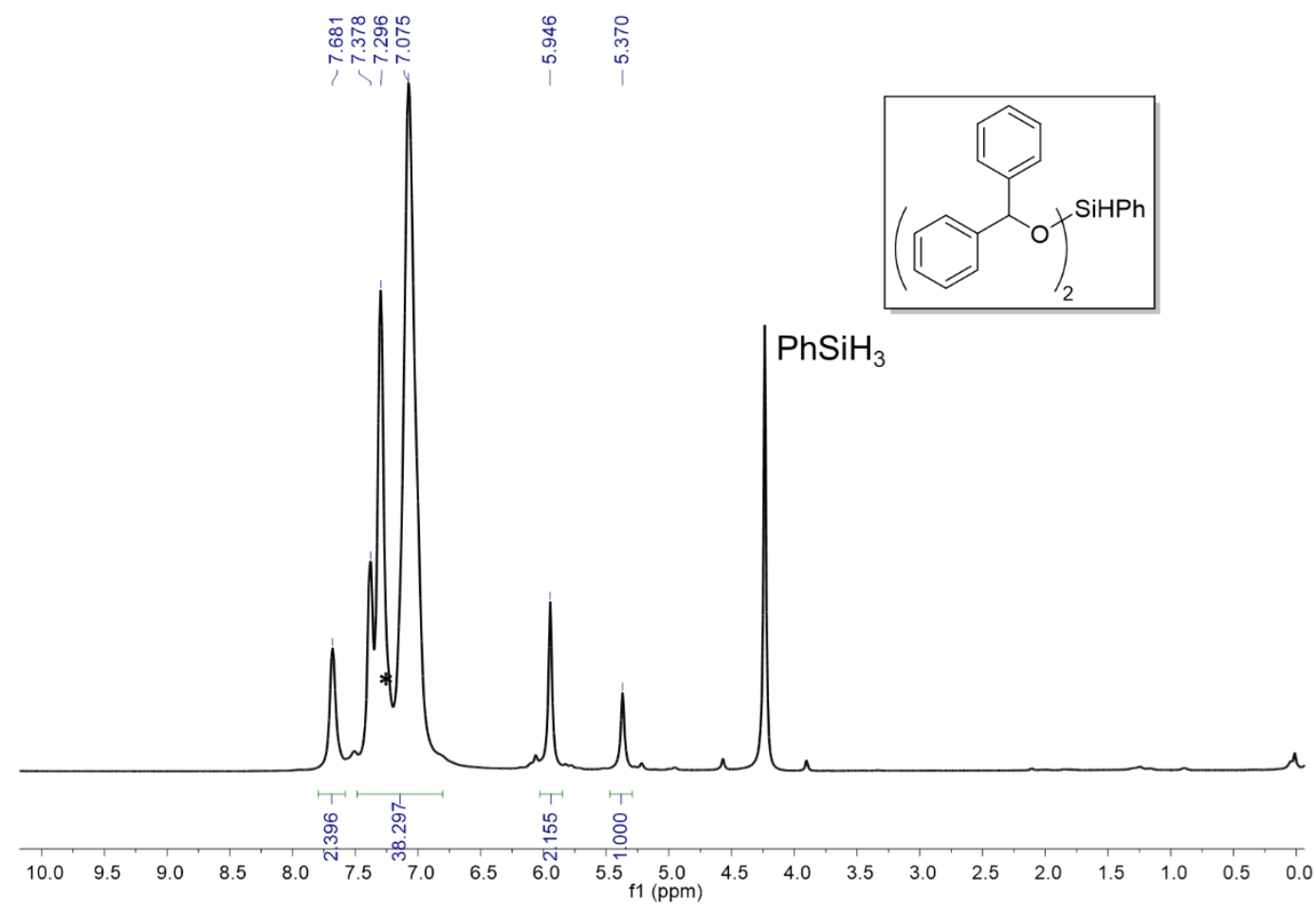

Figure S21 ${ }^{1} \mathrm{H}$ NMR spectrum ( $300 \mathrm{MHz}, \mathrm{C}_{6} \mathrm{D}_{6}$ ) of the catalytic hydrosilylation reaction of benzophenone with $\mathrm{PhSiH}_{3}$ catalyzed by complex 2 . The asterisk denotes the protio-residual resonance of $\mathrm{C}_{6} \mathrm{D}_{5} \mathrm{H}$ and all other unassigned peaks correspond to the silane $\mathrm{PhHSi}\left[\mathrm{OCH}(\mathrm{Ph})_{2}\right]_{2}$.

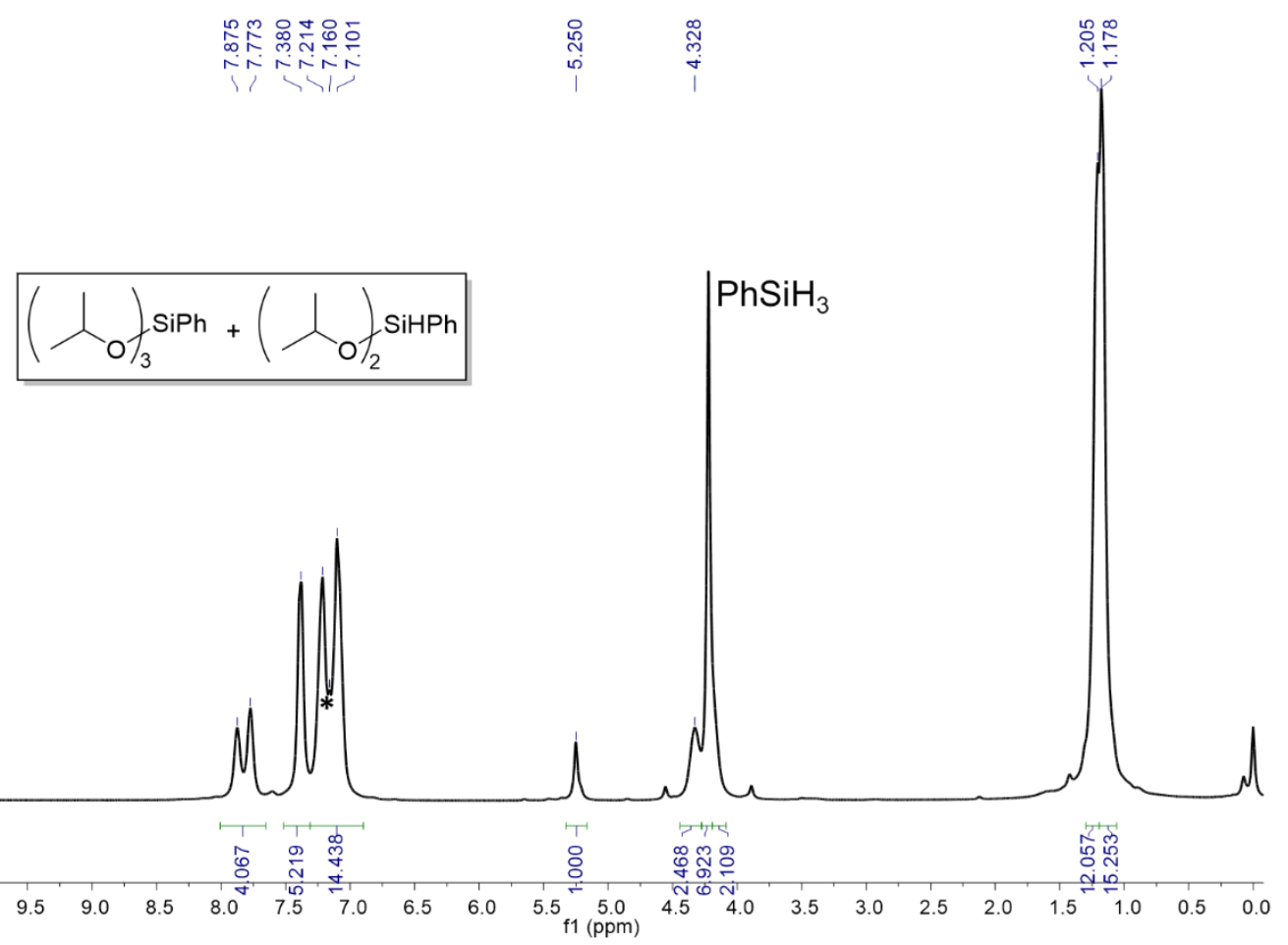

Figure S22 ${ }^{1} \mathrm{H}$ NMR spectrum $\left(300 \mathrm{MHz}, \mathrm{C}_{6} \mathrm{D}_{6}\right.$ ) of the catalytic hydrosilylation reaction of acetone with $\mathrm{PhSiH}_{3}$ catalyzed by complex 2 . The asterisk denotes the protio-residual resonance of $\mathrm{C}_{6} \mathrm{D}_{5} \mathrm{H}$ and all other unassigned peaks correspond to the mixture of silanes $\mathrm{PhSi}\left[\mathrm{OCH}\left(\mathrm{CH}_{3}\right)_{2}\right]_{3}$ and $\mathrm{PhHSi}\left[\mathrm{OCH}\left(\mathrm{CH}_{3}\right)_{2}\right]_{2}$. 


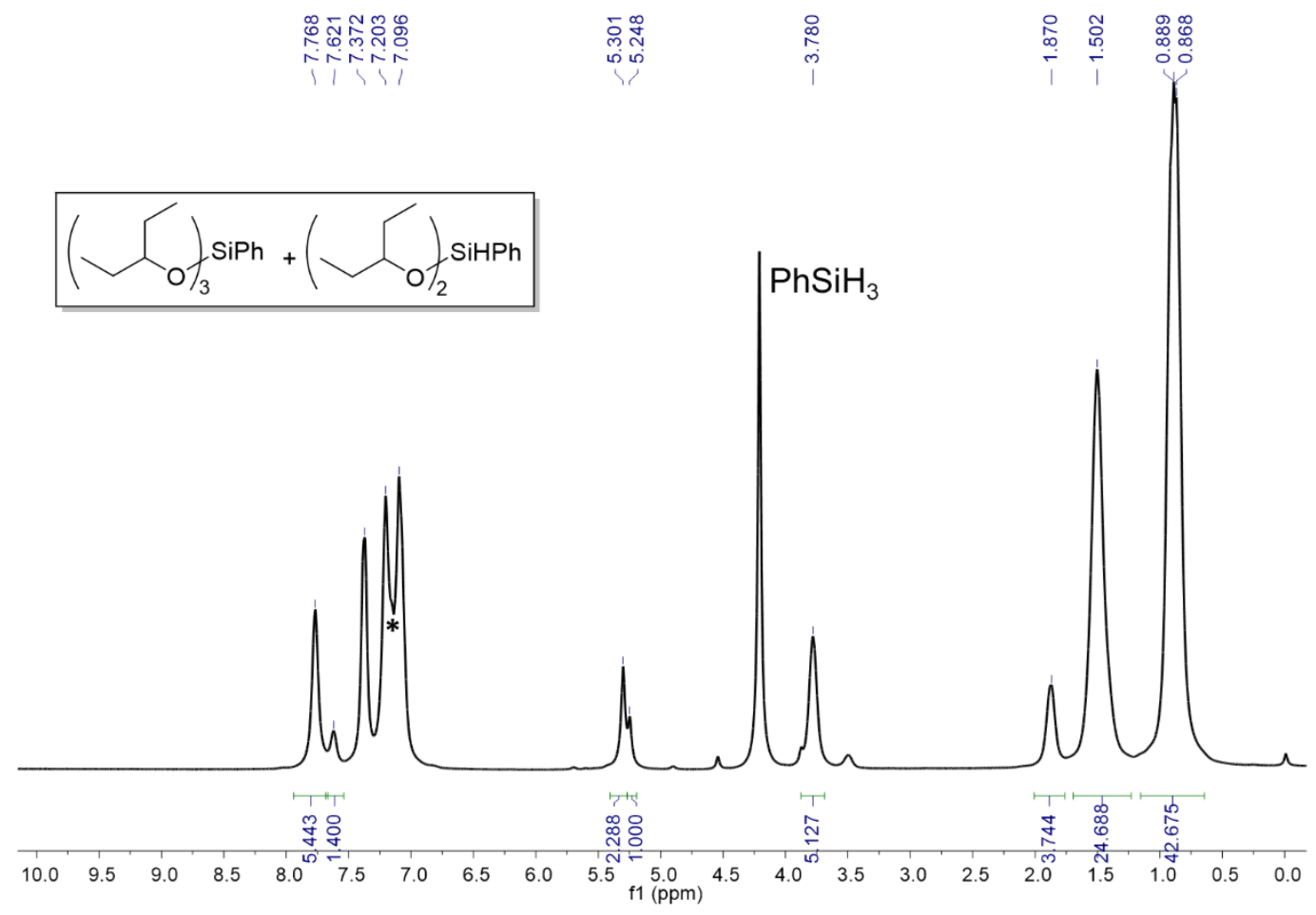

Figure S23 ${ }^{1} \mathrm{H}$ NMR spectrum (300 MHz, $\mathrm{C}_{6} \mathrm{D}_{6}$ ) of the catalytic hydrosilylation reaction of 3-pentanone with $\mathrm{PhSiH}_{3}$ catalyzed by complex 2 . The asterisk denotes the protio-residual resonance of $\mathrm{C}_{6} \mathrm{D}_{5} \mathrm{H}$ and all other unassigned peaks correspond to the mixture of silanes $\mathrm{PhSi}\left[\mathrm{OCH}\left(\mathrm{CH}_{2} \mathrm{CH}_{3}\right)_{2}\right]_{3}$ and $\mathrm{PhHSi}\left[\mathrm{OCH}\left(\mathrm{CH}_{2} \mathrm{CH}_{3}\right)_{2}\right]_{2}$. 


\section{NMR data of the alcoholic products obtained from hydrolysis of the silanes}

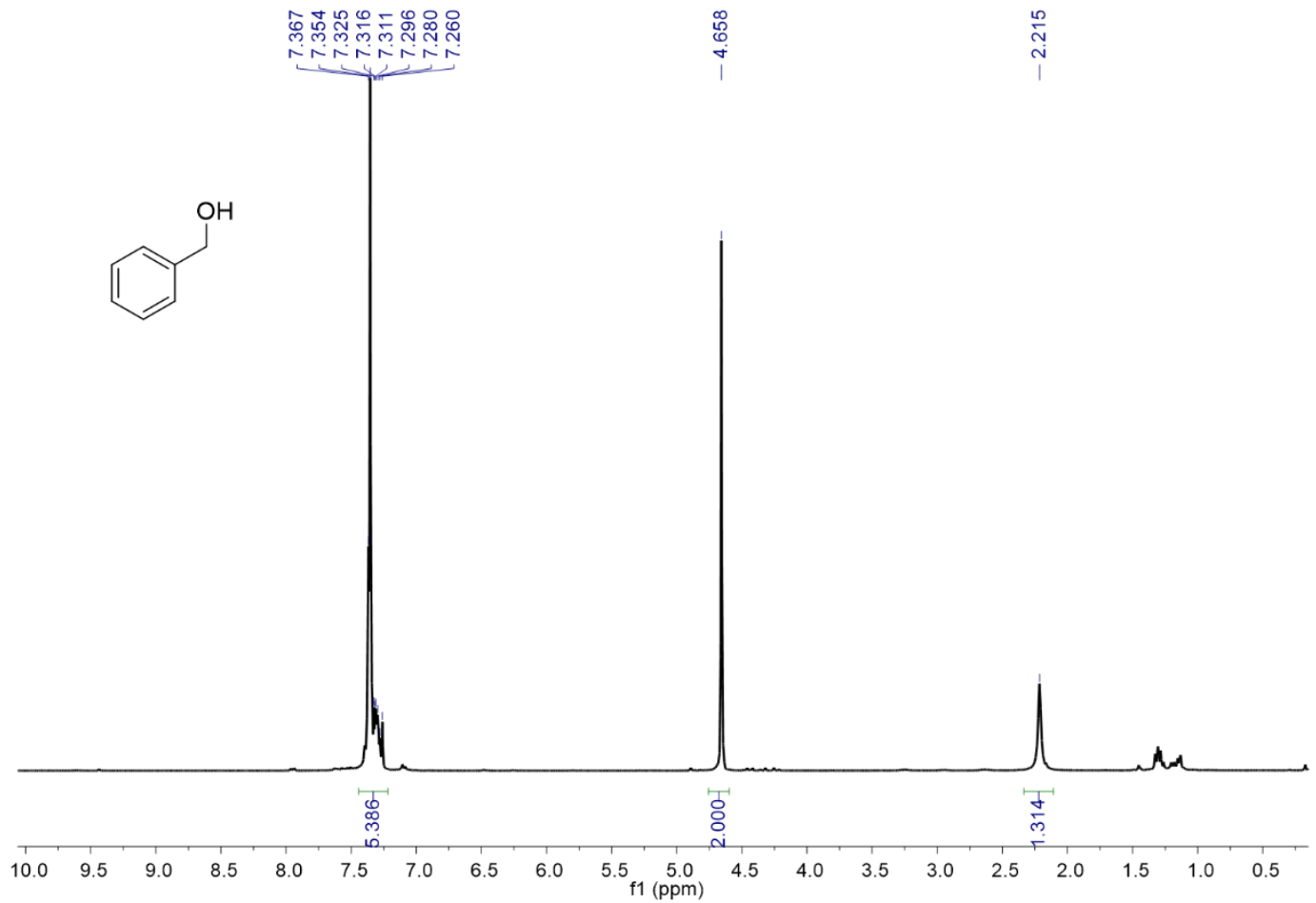

Figure S24 ${ }^{1} \mathrm{H}$ NMR spectrum $\left(300 \mathrm{MHz}, \mathrm{CDCl}_{3}\right)$ of phenylmethanol (benzyl alcohol) obtained from the hydrolysis of the silane $\mathrm{PhSi}\left(\mathrm{OCH}_{2} \mathrm{Ph}\right)_{3}$.

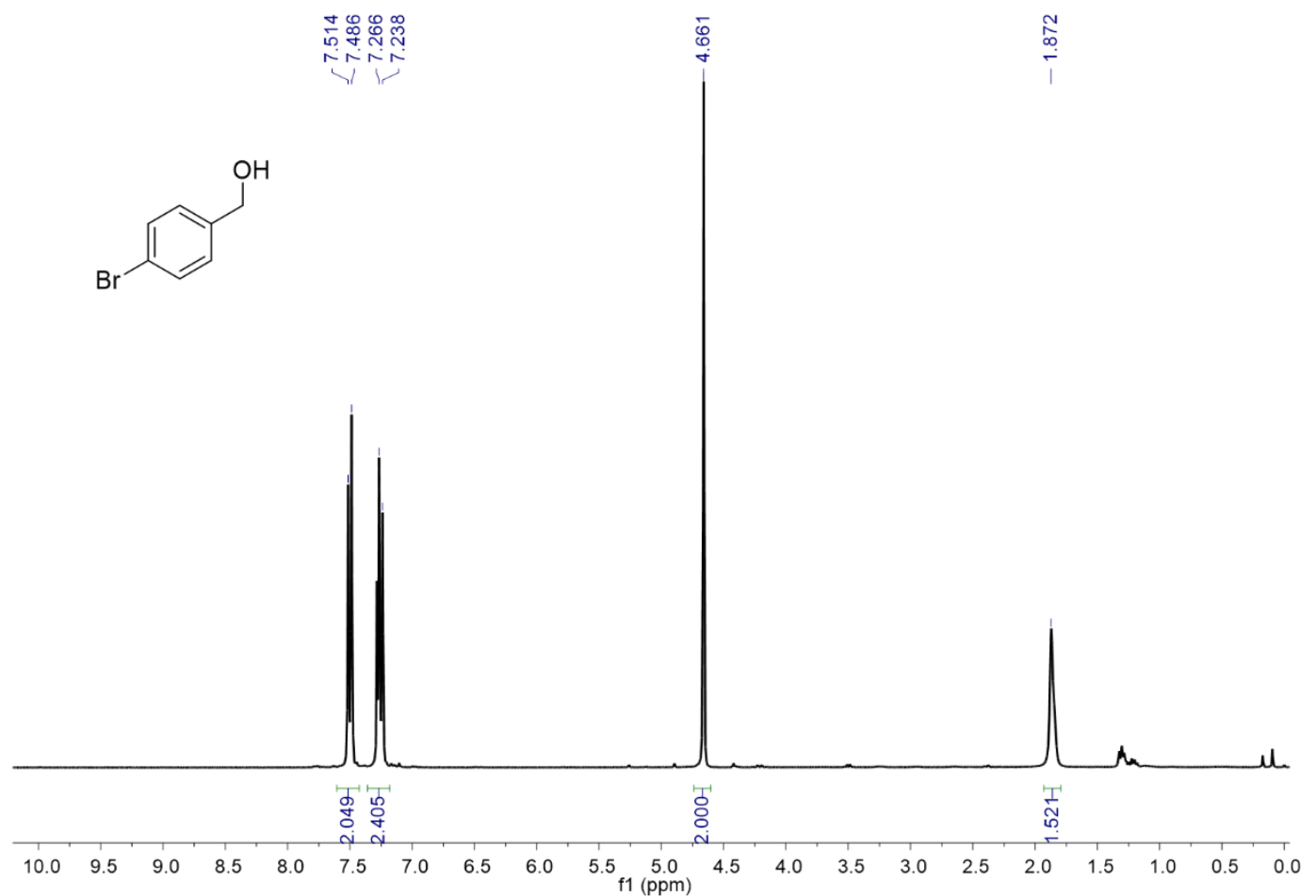

Figure $\mathbf{S 2 5}{ }^{1} \mathrm{H}$ NMR spectrum $\left(300 \mathrm{MHz}, \mathrm{CDCl}_{3}\right)$ of (4-bromophenyl)methanol obtained from the hydrolysis of the silane $\mathrm{PhSi}\left[\mathrm{OCH}_{2}\left(4-\mathrm{BrC}_{6} \mathrm{H}_{4}\right)\right]_{3}$. 


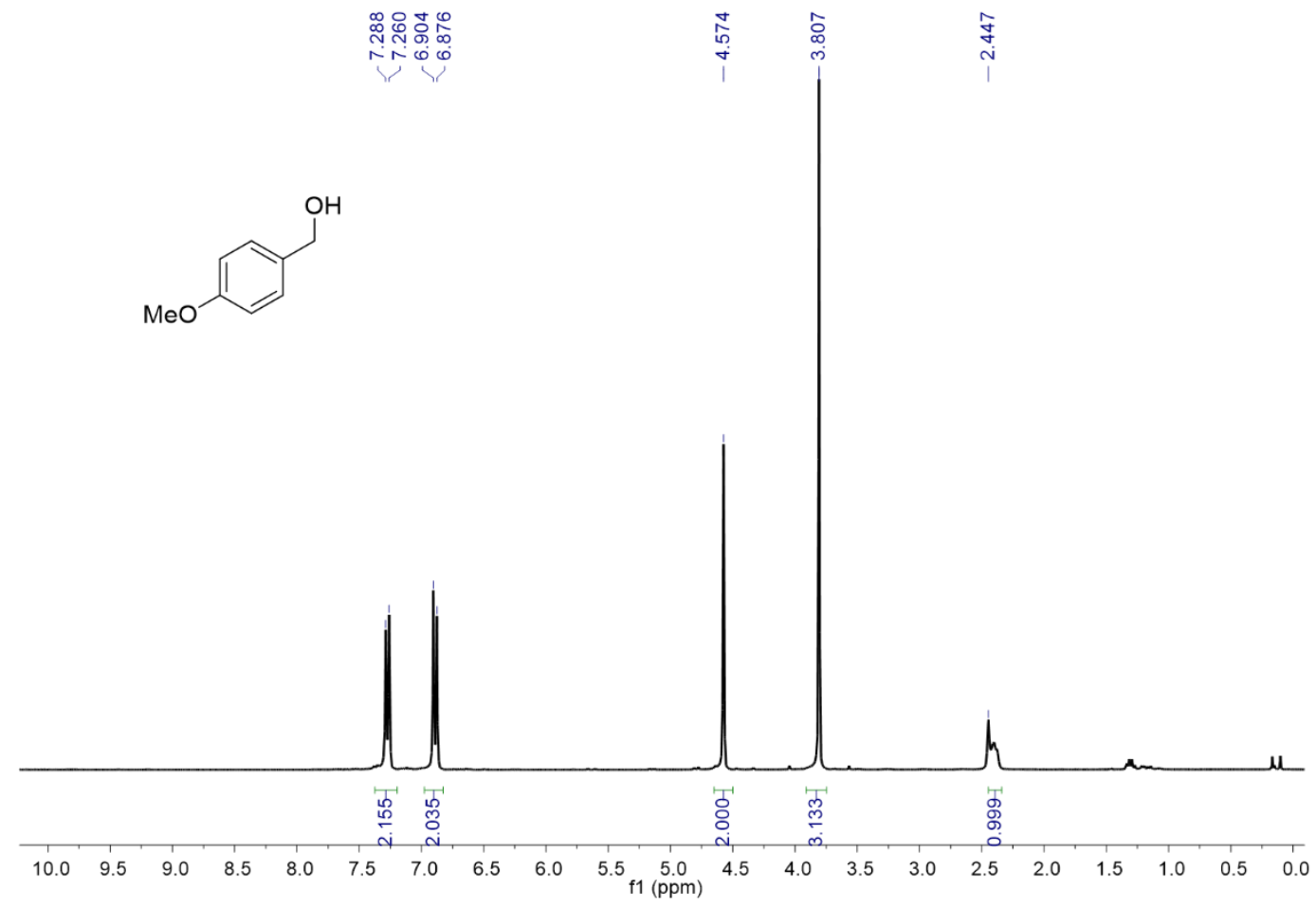

Figure S26 ${ }^{1} \mathrm{H}$ NMR spectrum (300 MHz, $\mathrm{CDCl}_{3}$ ) of (4-methoxyphenyl)methanol obtained from the hydrolysis of the silane $\mathrm{PhSi}_{2}\left[\mathrm{OCH}_{2}\left(4-\mathrm{OMeC}_{6} \mathrm{H}_{4}\right)\right]_{3}$.

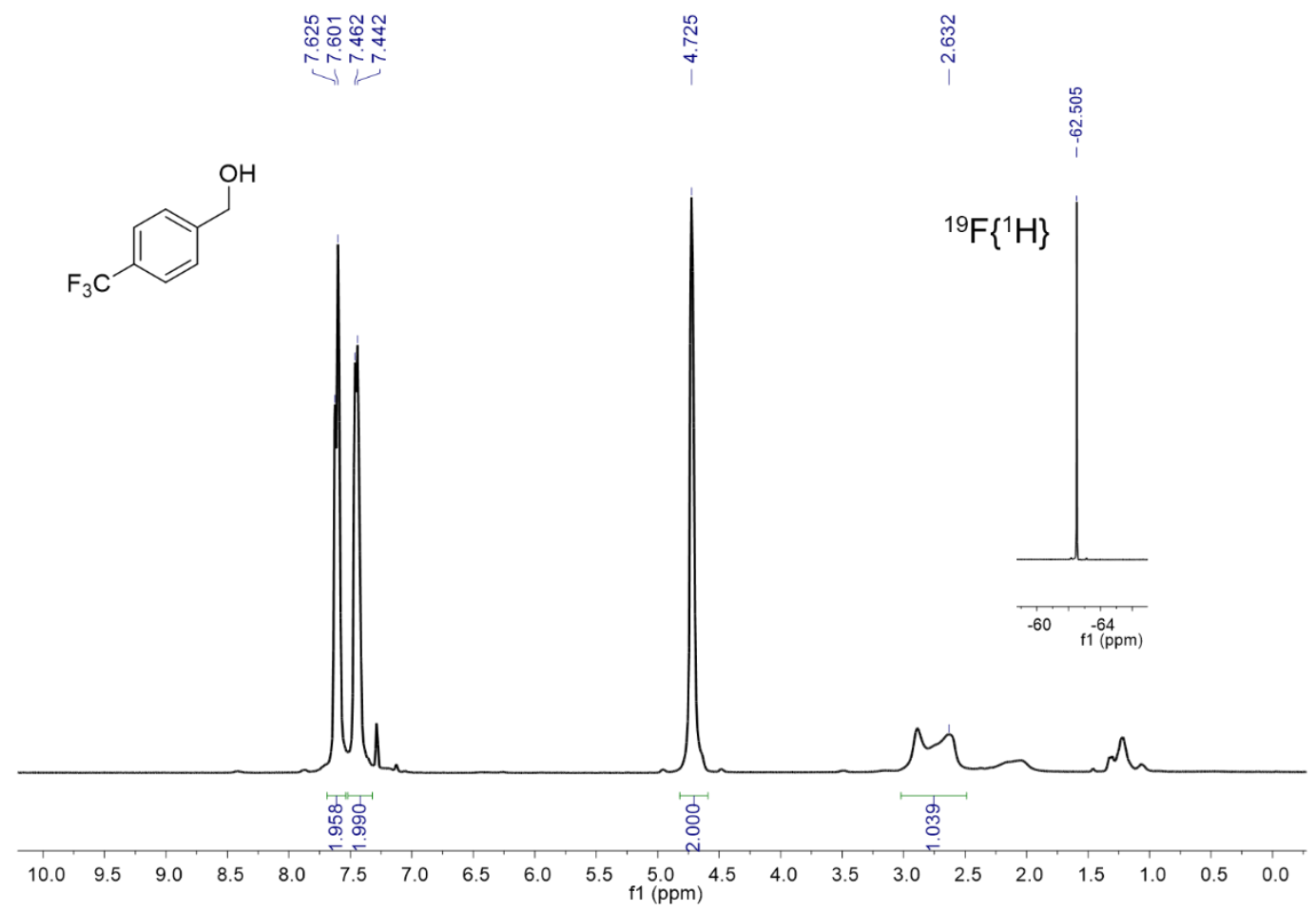

Figure S27 ${ }^{1} \mathrm{H}$ NMR spectrum (300 MHz, $\mathrm{CDCl}_{3}$ ) of (4-(trifluoromethyl)phenyl)methanol obtained from the hydrolysis of the silane $\mathrm{PhSi}\left[\mathrm{OCH}_{2}\left(4-\left(\mathrm{CF}_{3}\right) \mathrm{C}_{6} \mathrm{H}_{4}\right)\right]_{3}$. The inset shows the respective ${ }^{19} \mathrm{~F}\left\{{ }^{1} \mathrm{H}\right\}$ spectrum. 


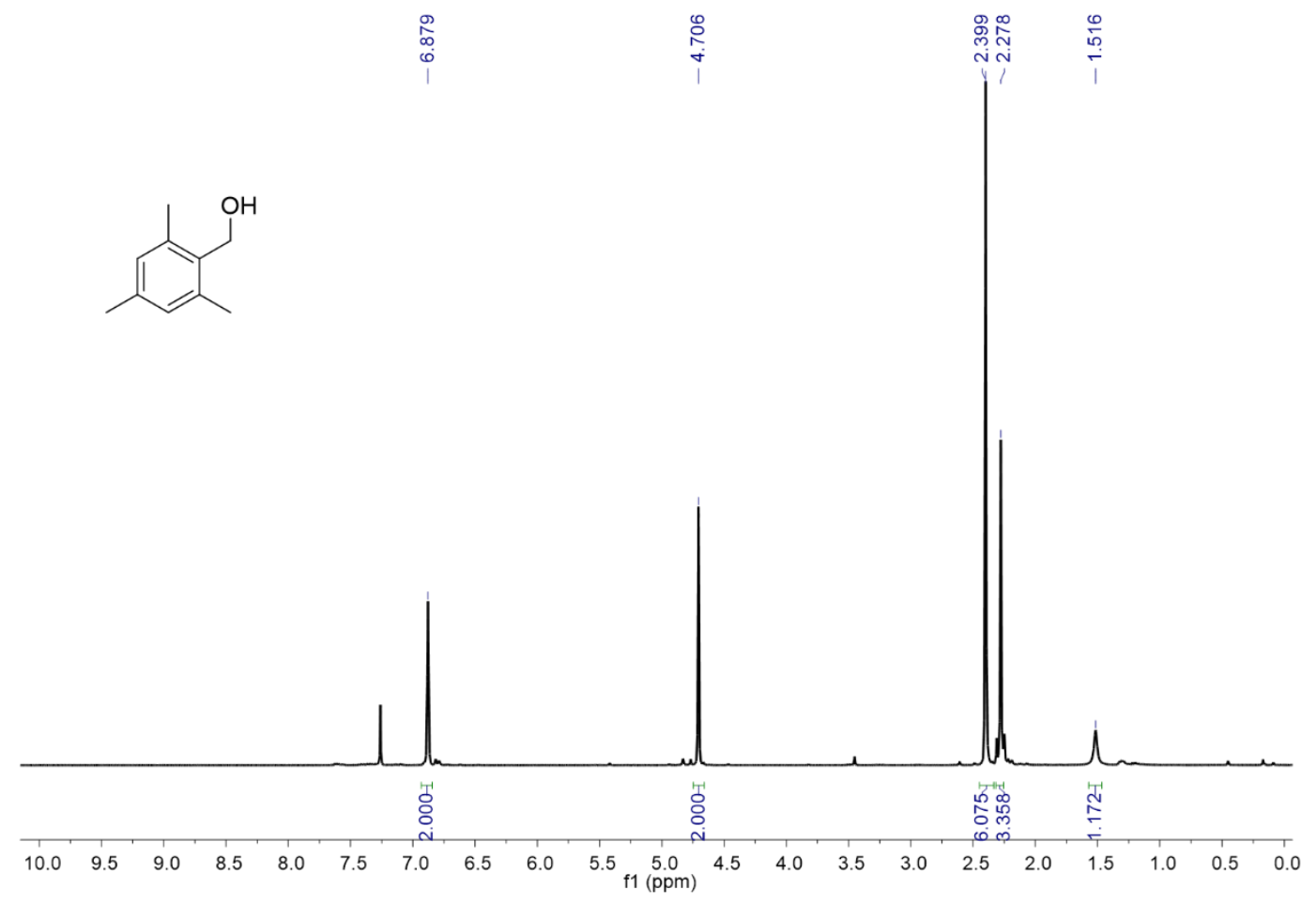

Figure $\mathbf{S 2 8}{ }^{1} \mathrm{H}$ NMR spectrum (300 MHz, $\left.\mathrm{CDCl}_{3}\right)$ of (2,4,6-trimethylphenyl)methanol obtained from the hydrolysis of the mixture of silanes $\mathrm{PhSi}\left\{\mathrm{OCH}_{2}\left[2,4,6-\left(\mathrm{CH}_{3}\right)_{3} \mathrm{C}_{6} \mathrm{H}_{2}\right]\right\}_{3}$ and $\left.\mathrm{PhHSi}_{\left\{\mathrm{OCH}_{2}\right.}\left[2,4,6-\left(\mathrm{CH}_{3}\right)_{3} \mathrm{C}_{6} \mathrm{H}_{2}\right]\right\}_{2}$.

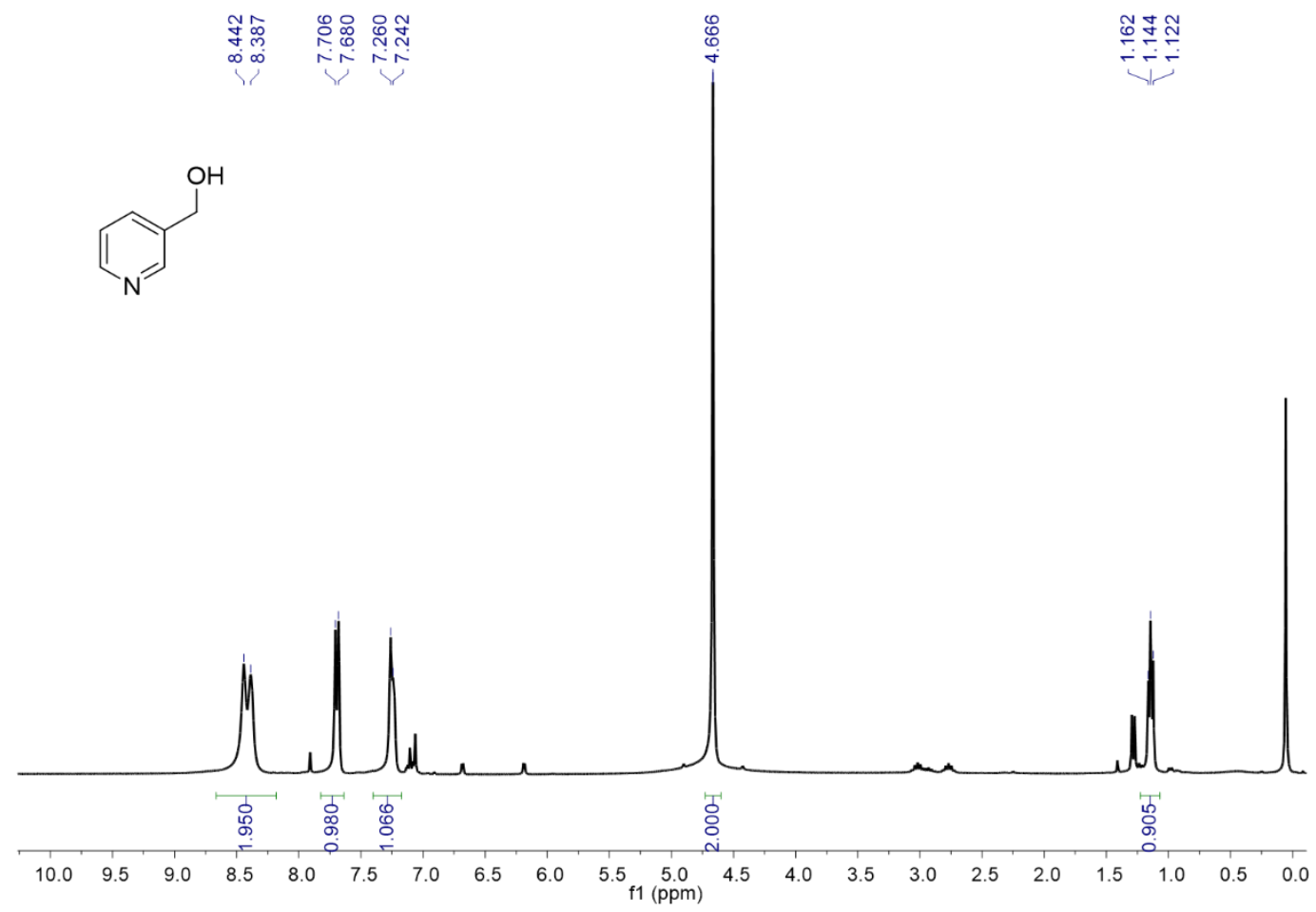

Figure S29 ${ }^{1} \mathrm{H}$ NMR spectrum $\left(300 \mathrm{MHz}, \mathrm{CDCl}_{3}\right)$ of pyridin-3-ylmethanolobtained from the hydrolysis of the silane $\mathrm{PhSi}_{2}\left[\mathrm{OCH}_{2}\left(\mathrm{C}_{5} \mathrm{H}_{4} \mathrm{~N}\right)\right]_{3}$. 


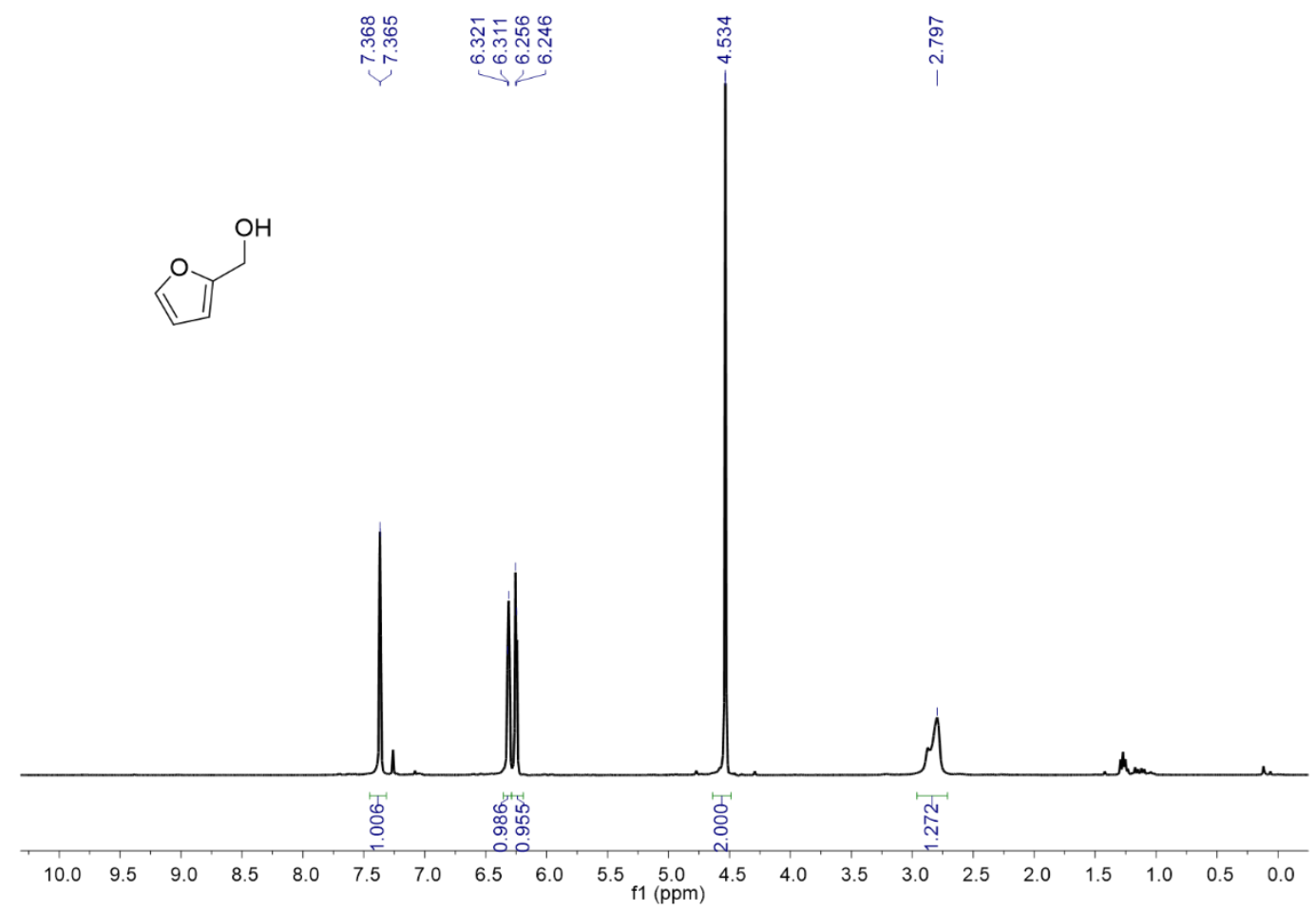

Figure S30 ${ }^{1} \mathrm{H}$ NMR spectrum ( $300 \mathrm{MHz}, \mathrm{CDCl}_{3}$ ) of furan-2-ylmethanol obtained from the hydrolysis of the silane $\mathrm{PhSi}_{0}\left[\mathrm{OCH}_{2}\left(\mathrm{C}_{4} \mathrm{H}_{3} \mathrm{O}\right)\right]_{3}$.

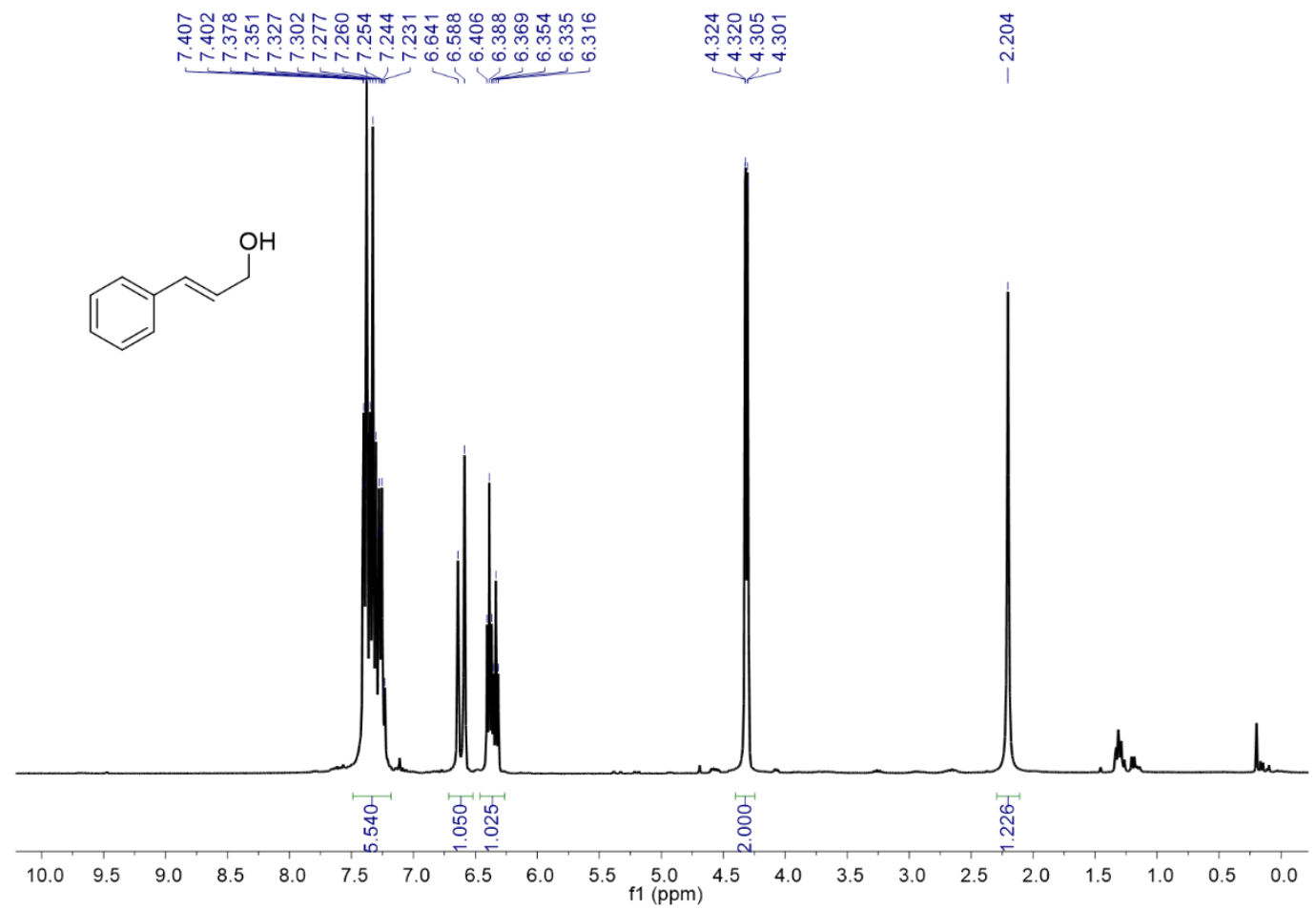

Figure $\mathbf{S 3 1}{ }^{1} \mathrm{H}$ NMR spectrum (300 MHz, $\mathrm{CDCl}_{3}$ ) of $(E)$-3-phenylprop-2-en-1-ol obtained from the hydrolysis of the silane $\mathrm{PhSi}\left(\mathrm{OCH}_{2} \mathrm{CHCHPh}\right)_{3}$. 


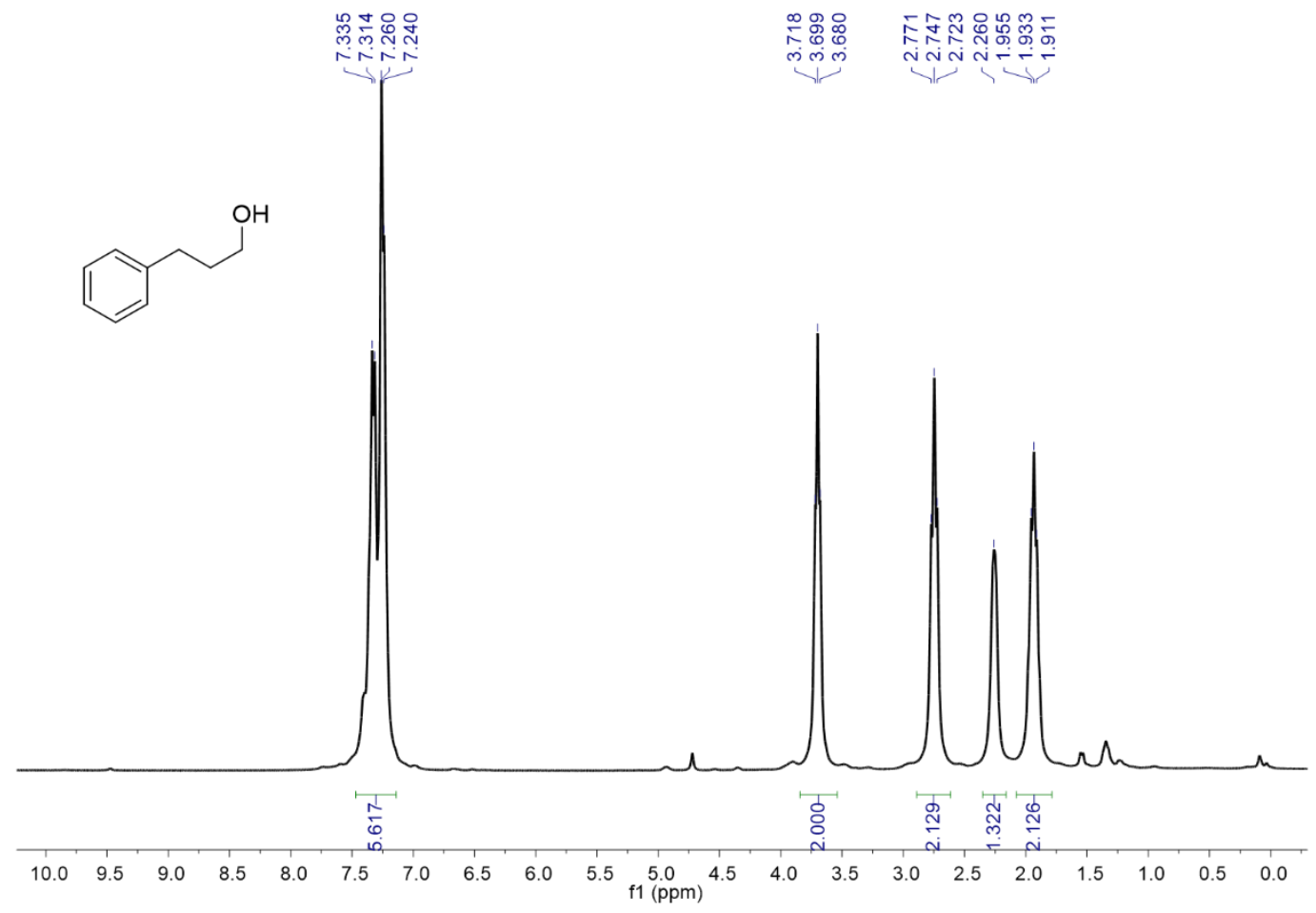

Figure S32 ${ }^{1} \mathrm{H}$ NMR spectrum (300 MHz, $\mathrm{CDCl}_{3}$ ) of 3-phenylpropan-1-ol obtained from the hydrolysis of the silane $\mathrm{PhSi}\left(\mathrm{OCH}_{2} \mathrm{CH}_{2} \mathrm{CH}_{2} \mathrm{Ph}\right)_{3}$.

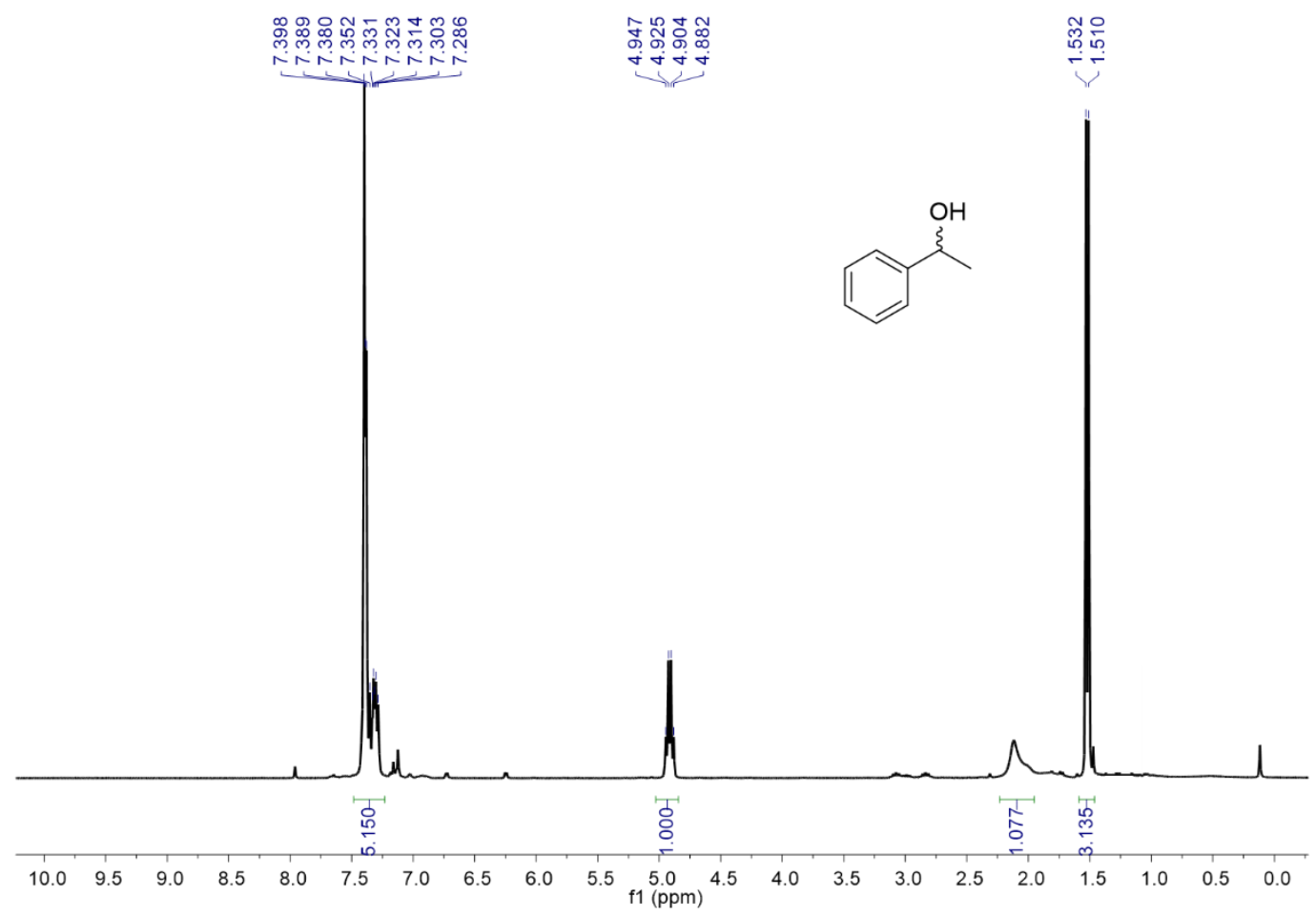

Figure $\mathbf{S 3 3}{ }^{1} \mathrm{H}$ NMR spectrum (300 MHz, $\mathrm{CDCl}_{3}$ ) of the mixture of the stereoisomers of 1-phenylethan-1-ol obtained from the hydrolysis of the mixture of silanes $\mathrm{PhSi}\left[\mathrm{OCH}\left(\mathrm{CH}_{3}\right) \mathrm{Ph}\right]_{3}$ and $\mathrm{PhHSi}\left[\mathrm{OCH}\left(\mathrm{CH}_{3}\right) \mathrm{Ph}\right]_{2}$. 


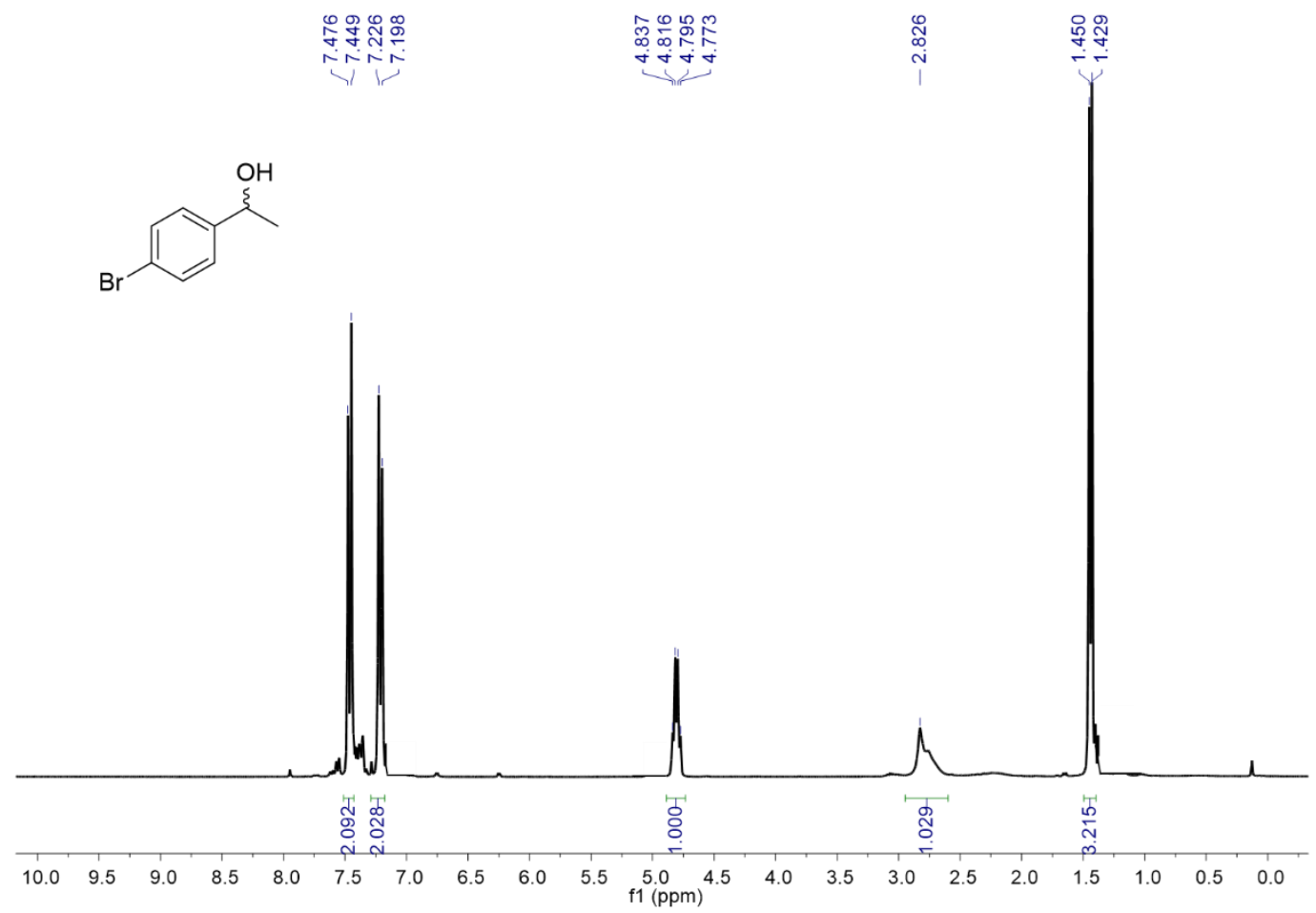

Figure $\mathbf{S 3 4}{ }^{1} \mathrm{H}$ NMR spectrum $\left(300 \mathrm{MHz}, \mathrm{CDCl}_{3}\right.$ ) of the mixture of the stereoisomers of 1-(4-bromophenyl)ethan-1-ol obtained from the hydrolysis of the mixture of silanes $\mathrm{PhSi}\left[\mathrm{OCH}\left(\mathrm{CH}_{3}\right)\left(4-\mathrm{BrC}_{6} \mathrm{H}_{4}\right)\right]_{3}$ and $\mathrm{PhHSi}\left[\mathrm{OCH}\left(\mathrm{CH}_{3}\right)\left(4-\mathrm{BrC}_{6} \mathrm{H}_{4}\right)\right]_{2}$.

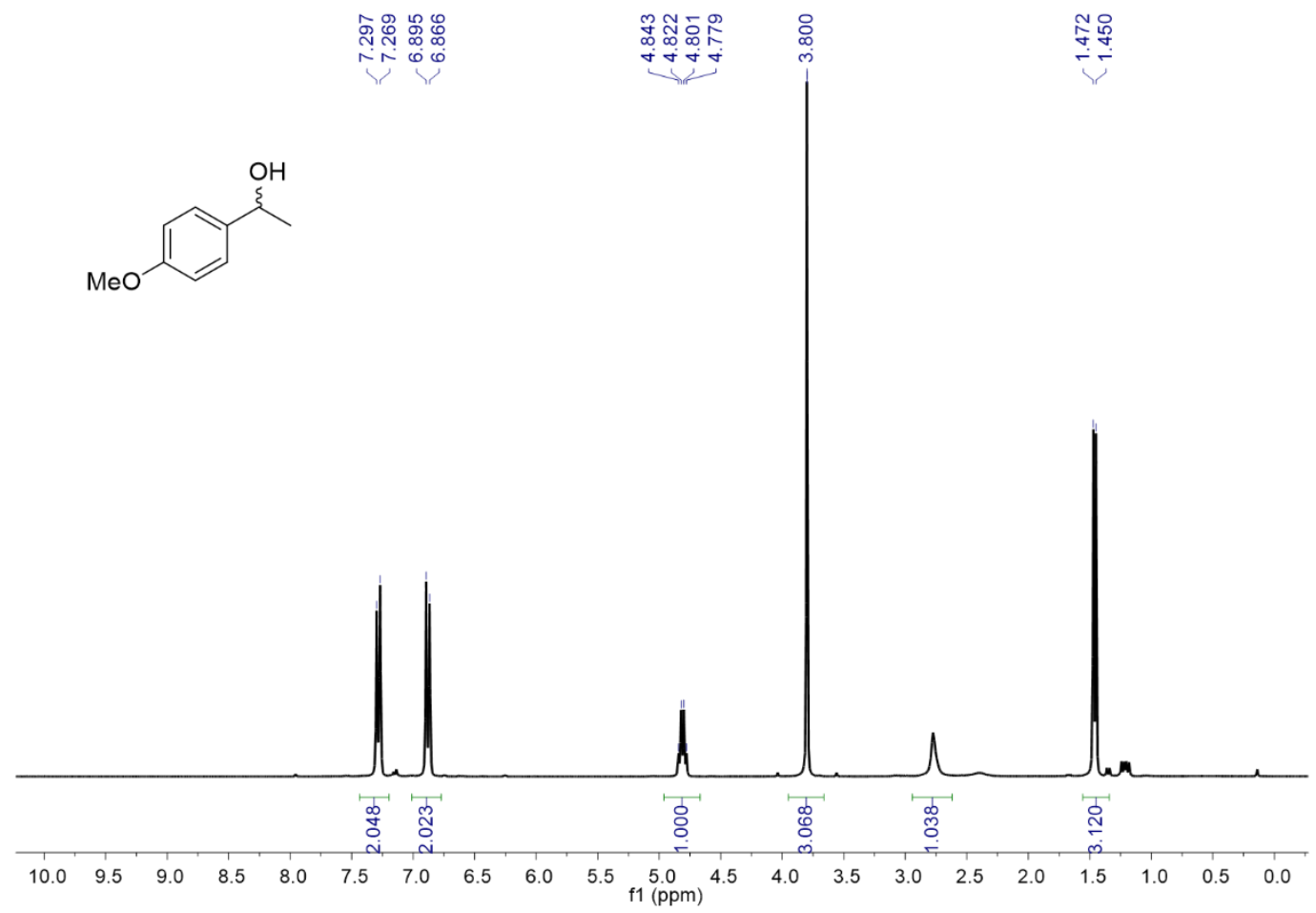

Figure S35 ${ }^{1} \mathrm{H}$ NMR spectrum (300 MHz, $\mathrm{CDCl}_{3}$ ) of the mixture of the stereoisomers of 1-(4-methoxyphenyl)ethan-1-ol obtained from the hydrolysis of the mixture of silanes $\mathrm{PhSi}\left[\mathrm{OCH}\left(\mathrm{CH}_{3}\right)\left(4-\mathrm{OMeC}_{6} \mathrm{H}_{4}\right)\right]_{3}$ and $\mathrm{PhHSi}\left[\mathrm{OCH}\left(\mathrm{CH}_{3}\right)\left(4-\mathrm{OMeC}_{6} \mathrm{H}_{4}\right)\right]_{2}$. 


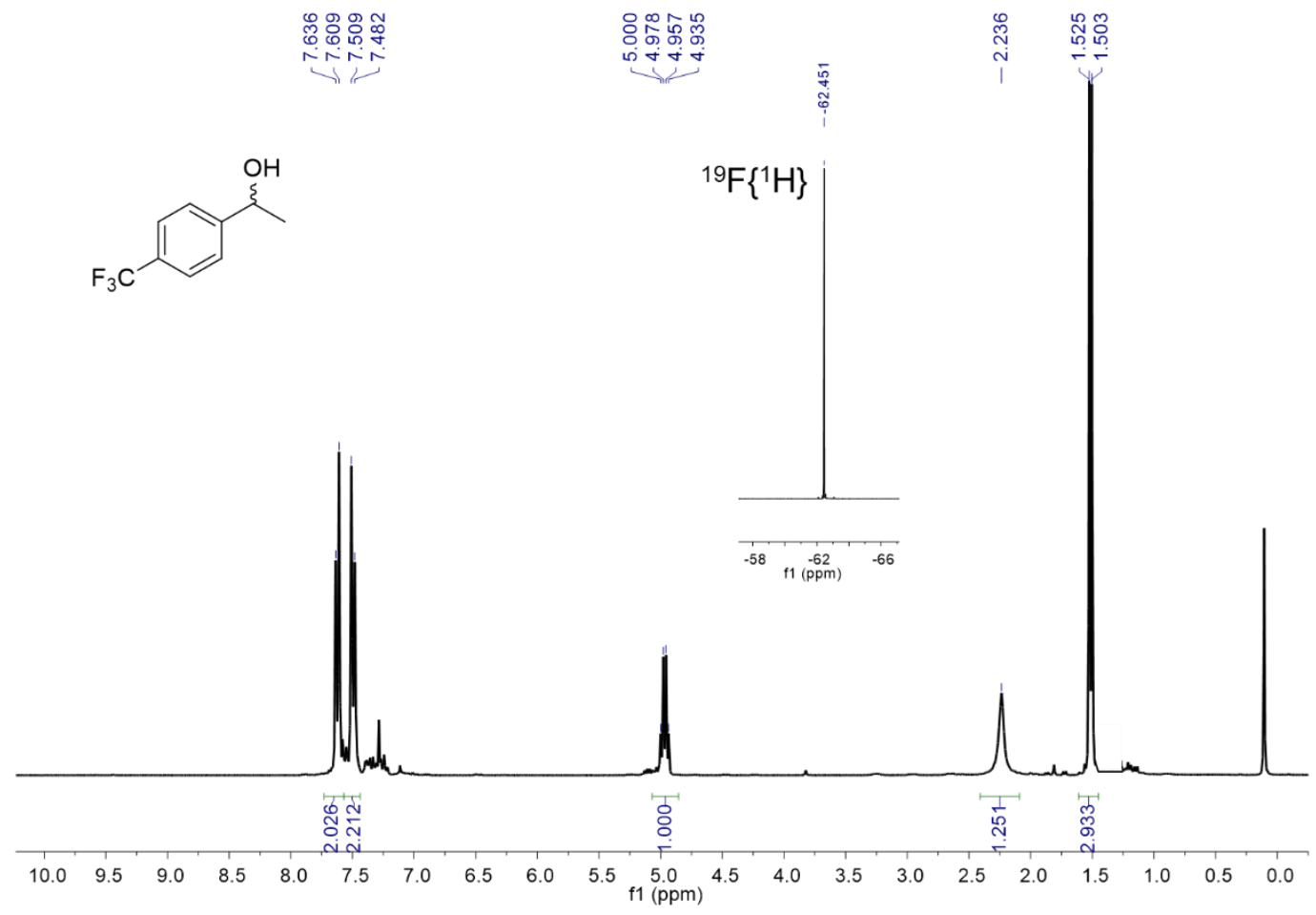

Figure S36 ${ }^{1} \mathrm{H}$ NMR spectrum $\left(300 \mathrm{MHz}, \mathrm{CDCl}_{3}\right)$ of the mixture of the stereoisomers of 1-(4-(trifluoromethyl)phenyl)ethan-1-ol obtained from the hydrolysis of the mixture of silanes $\mathrm{PhSi}\left\{\mathrm{OCH}\left(\mathrm{CH}_{3}\right)\left[4-\left(\mathrm{CF}_{3}\right) \mathrm{C}_{6} \mathrm{H}_{4}\right]\right\}_{3}$ and $\mathrm{PhHSi}\left\{\mathrm{OCH}\left(\mathrm{CH}_{3}\right)\left[4-\left(\mathrm{CF}_{3}\right) \mathrm{C}_{6} \mathrm{H}_{4}\right]\right\}_{2}$. The inset shows the respective ${ }^{19} \mathrm{~F}\left\{{ }^{1} \mathrm{H}\right\}$ spectrum.

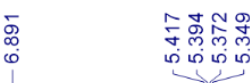

규

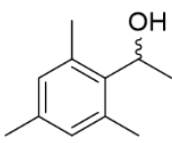

아
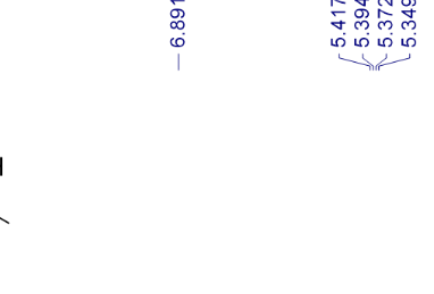


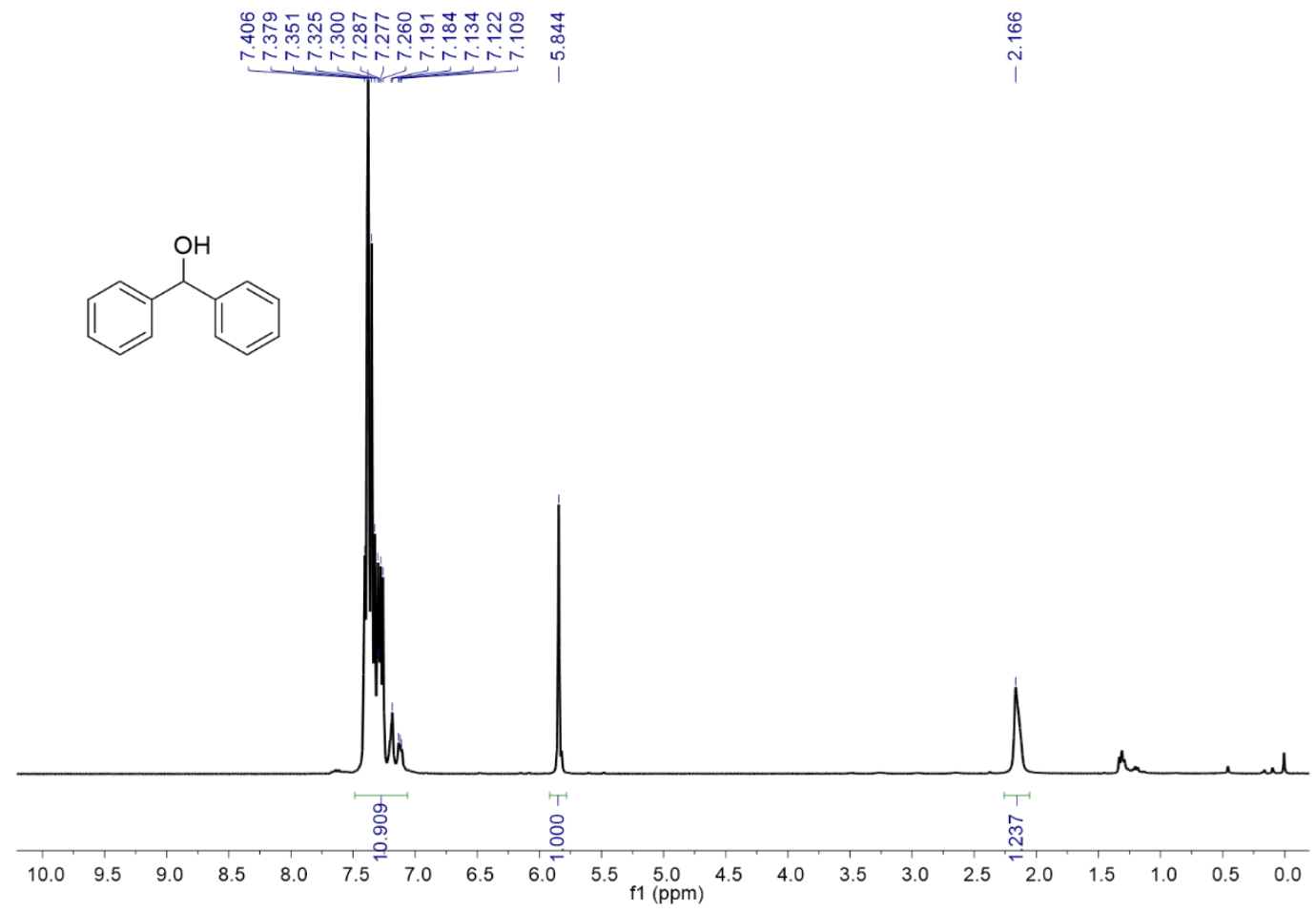

Figure S38 ${ }^{1} \mathrm{H}$ NMR spectrum $\left(300 \mathrm{MHz}, \mathrm{CDCl}_{3}\right.$ ) of diphenylmethanol obtained from the hydrolysis of the silane $\mathrm{PhHSi}\left[\mathrm{OCH}(\mathrm{Ph})_{2}\right]_{2}$. 


\section{NMR data for the Hammett analysis}

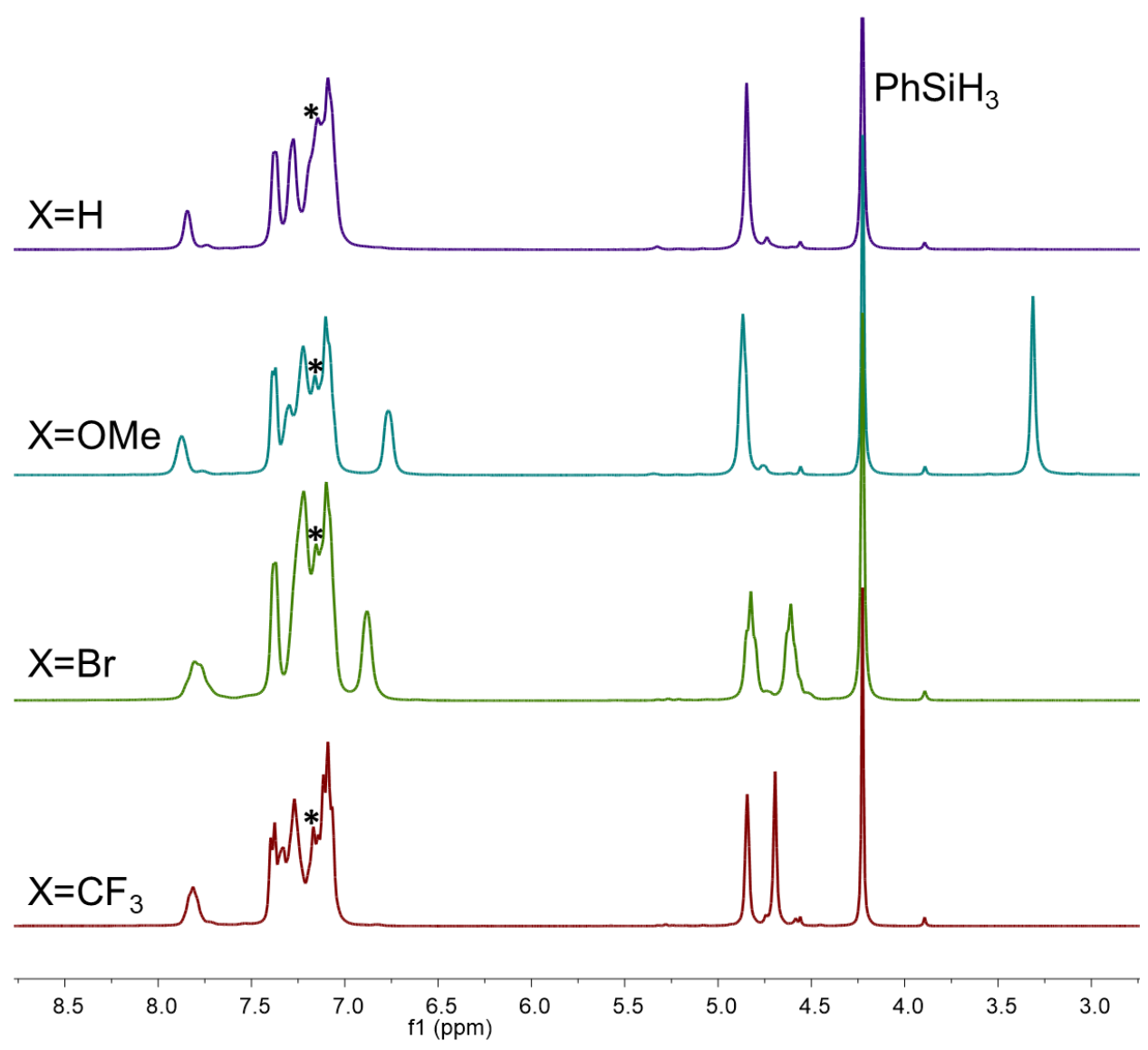

Figure S39 Stacking of the ${ }^{1} \mathrm{H}$ NMR spectra $\left(300 \mathrm{MHz}, \mathrm{C}_{6} \mathrm{D}_{6}\right)$ of the catalytic hydrosilylation competition experiments of equimolar amounts of benzaldehyde and a para-X-substituted benzaldehyde with $\mathrm{PhSiH}_{3}$ catalyzed by complex 2 . The asterisks denote the protio-residual resonance of $\mathrm{C}_{6} \mathrm{D}_{5} \mathrm{H}$ and all other unassigned peaks correspond to the respective mixture of silanes.
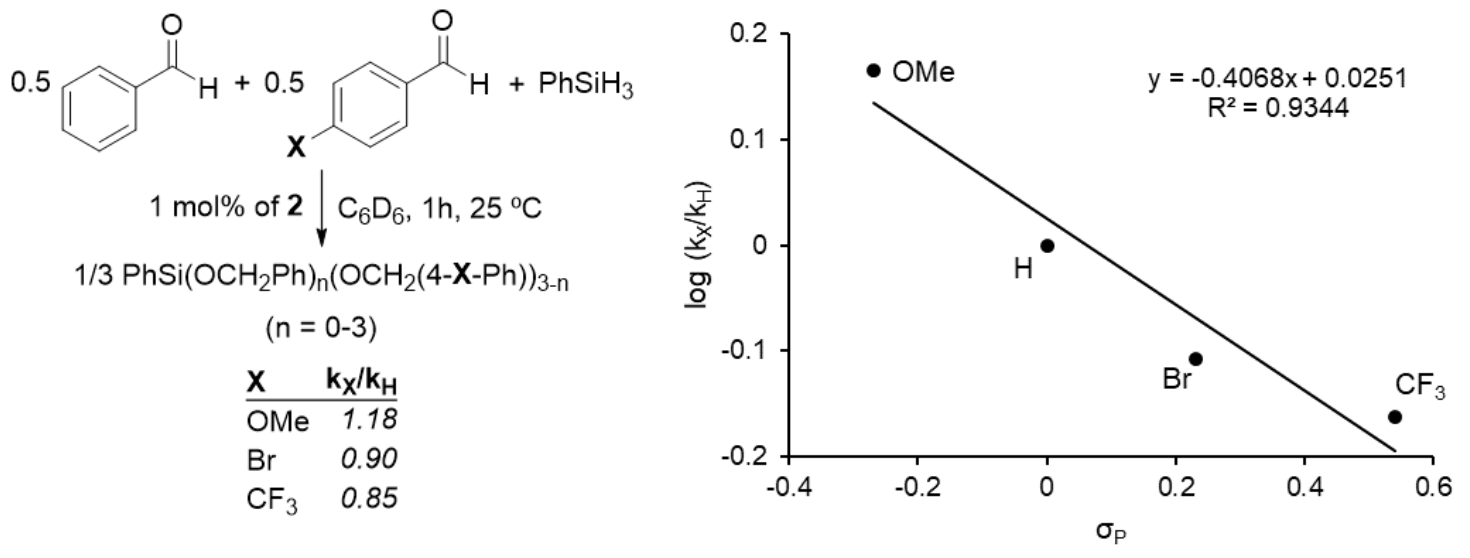

Figure S40 Hammett plot for the catalytic hydrosilylation of different benzaldehydes with $\mathrm{PhSiH}_{3}$ catalyzed by complex 2 . Values of kx/kH were determined by ${ }^{1} \mathrm{H}$ NMR spectroscopy. ${ }^{1}$

\footnotetext{
${ }^{1}$ Hansch, C.; Leo, A.; Taft, R. W. A survey of Hammett substituent constants and resonance and field parameters. Chem. Rev. 1991, 91, 165-195.
} 


\section{NMR scale and GC-TCD mechanistic investigations}

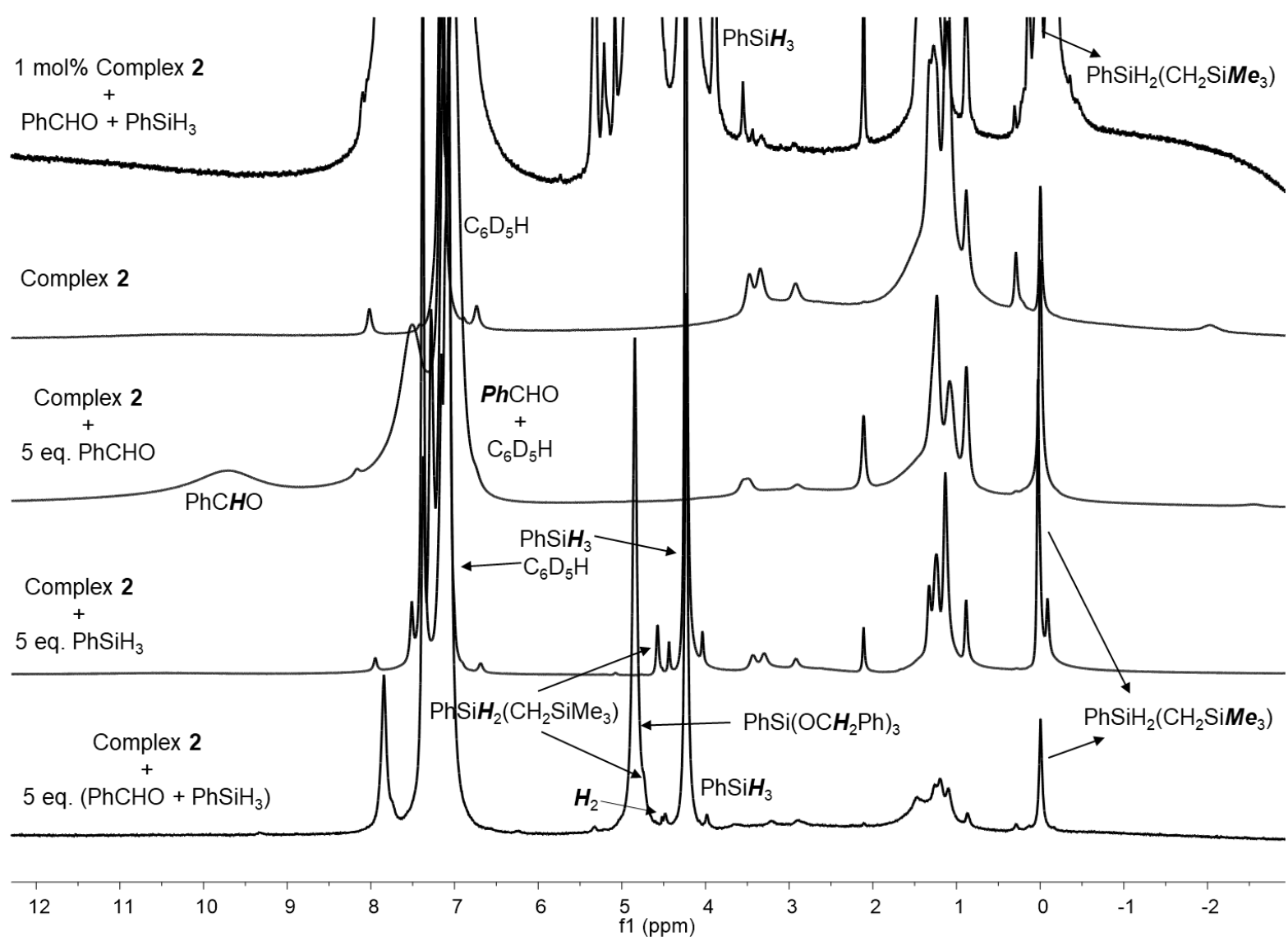

Figure S41 Stacking of the diamagnetic regions of the ${ }^{1} \mathrm{H}$ NMR spectra $\left(300 \mathrm{MHz}, \mathrm{C}_{6} \mathrm{D}_{6}\right)$ of a catalytic hydrosilylation of benzaldehyde (first spectrum - top), complex 2 (second spectrum), the reaction of complex 2 with five equivalents of benzaldehyde ( $\mathrm{PhCHO}$ ) (third spectrum), the reaction of complex 2 with five equivalents of $\mathrm{PhSiH}_{3}$ (fourth spectrum) and the reaction of complex 2 with five equivalents of $\mathrm{PhSiH}_{3}$ and five equivalents of benzaldehyde (fifth spectrum, bottom). 


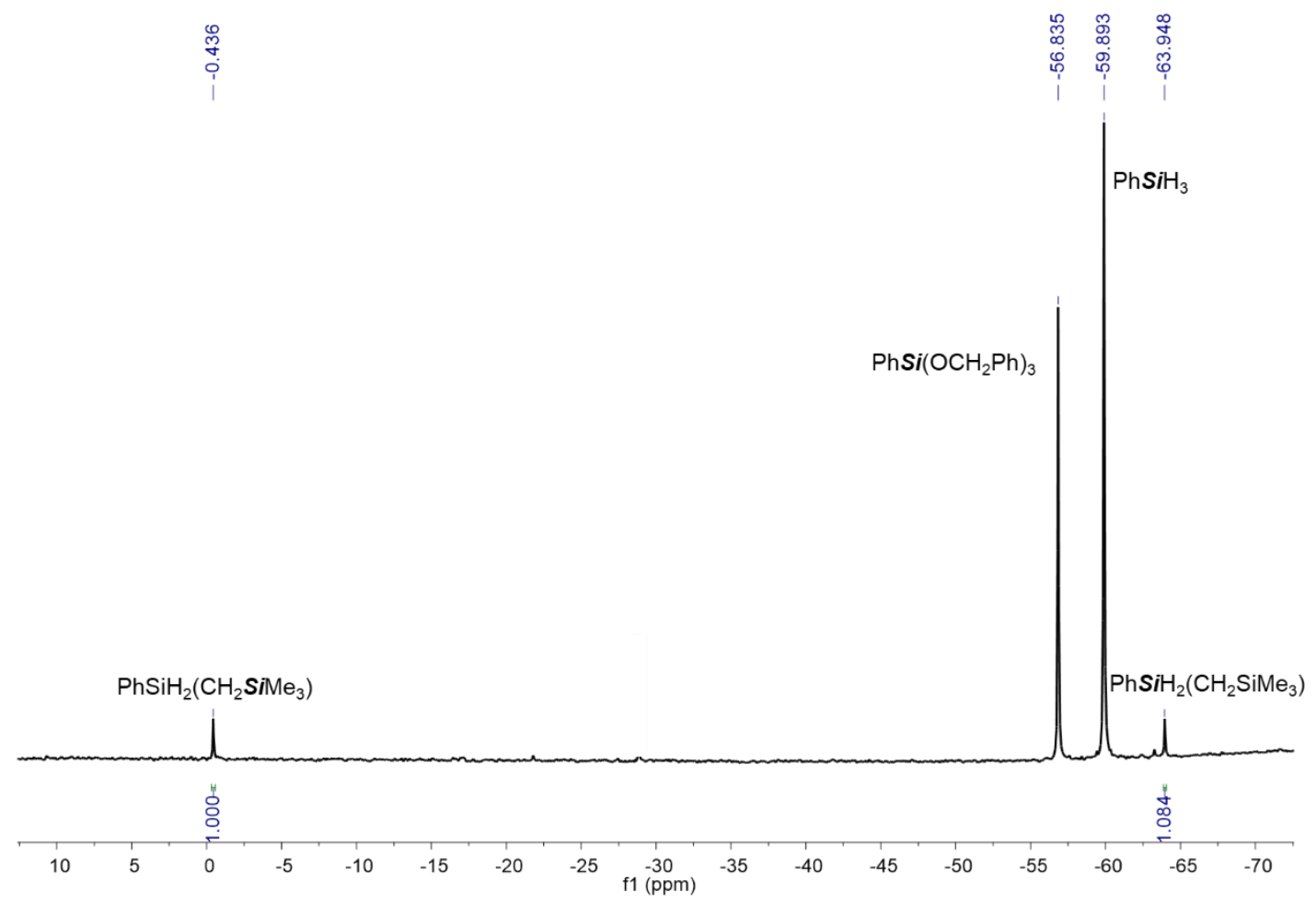

Figure $\mathbf{S 4 2}{ }^{29} \mathrm{Si}\left\{{ }^{1} \mathrm{H}\right\}$ NMR (79.53 MHz, $\left.\mathrm{C}_{6} \mathrm{D}_{6}\right)$ spectrum obtained from the reaction of complex 2 with five equivalents of $\mathrm{PhSiH}_{3}$ and five equivalents of benzaldehyde.

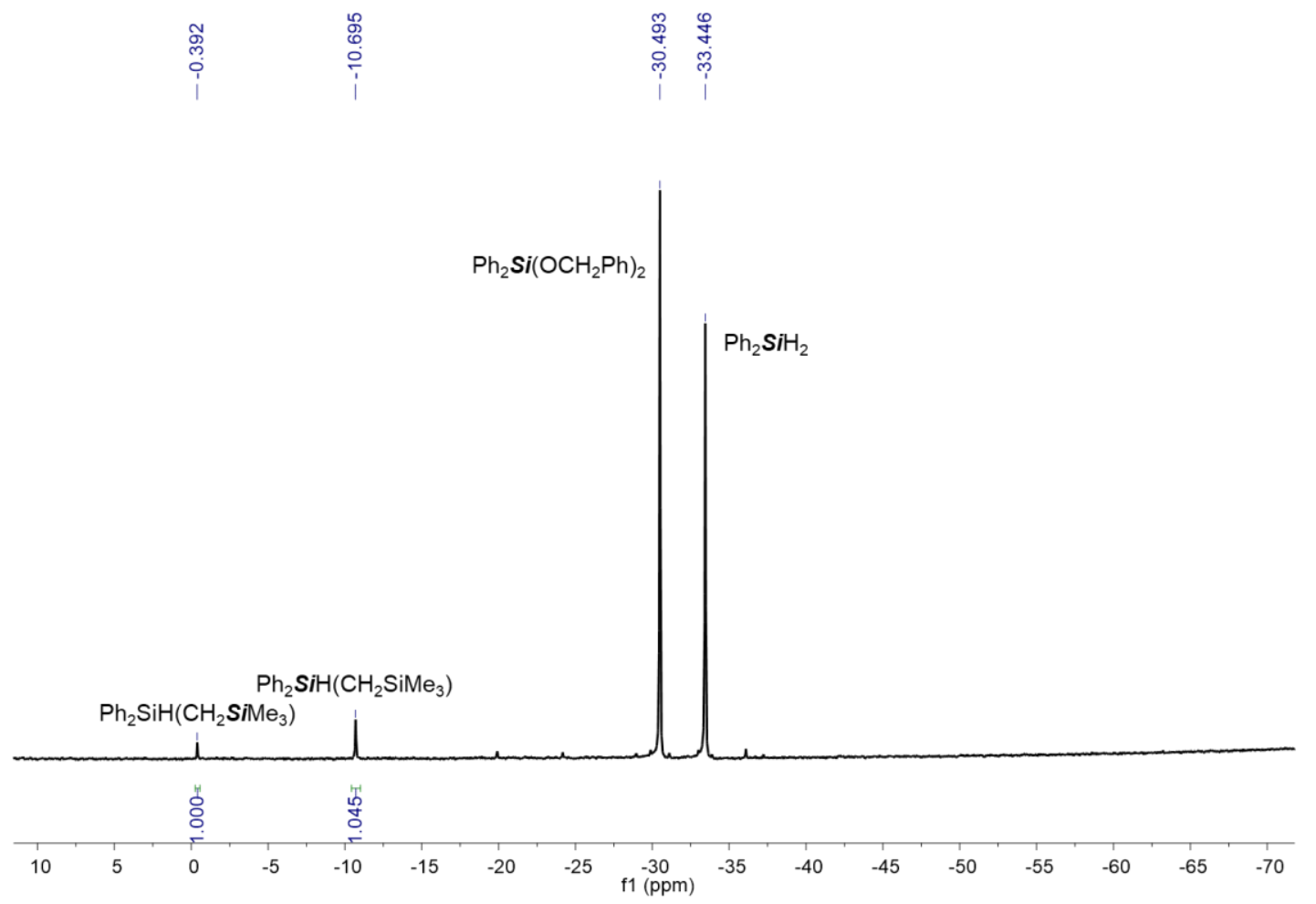

Figure $\mathbf{S 4 3}{ }^{29} \mathrm{Si}\left\{{ }^{1} \mathrm{H}\right\}$ NMR $\left(79.53 \mathrm{MHz}, \mathrm{C}_{6} \mathrm{D}_{6}\right)$ spectrum obtained from the reaction of complex $\mathbf{2}$ with five equivalents of $\mathrm{Ph}_{2} \mathrm{SiH}_{2}$ and five equivalents of benzaldehyde. 


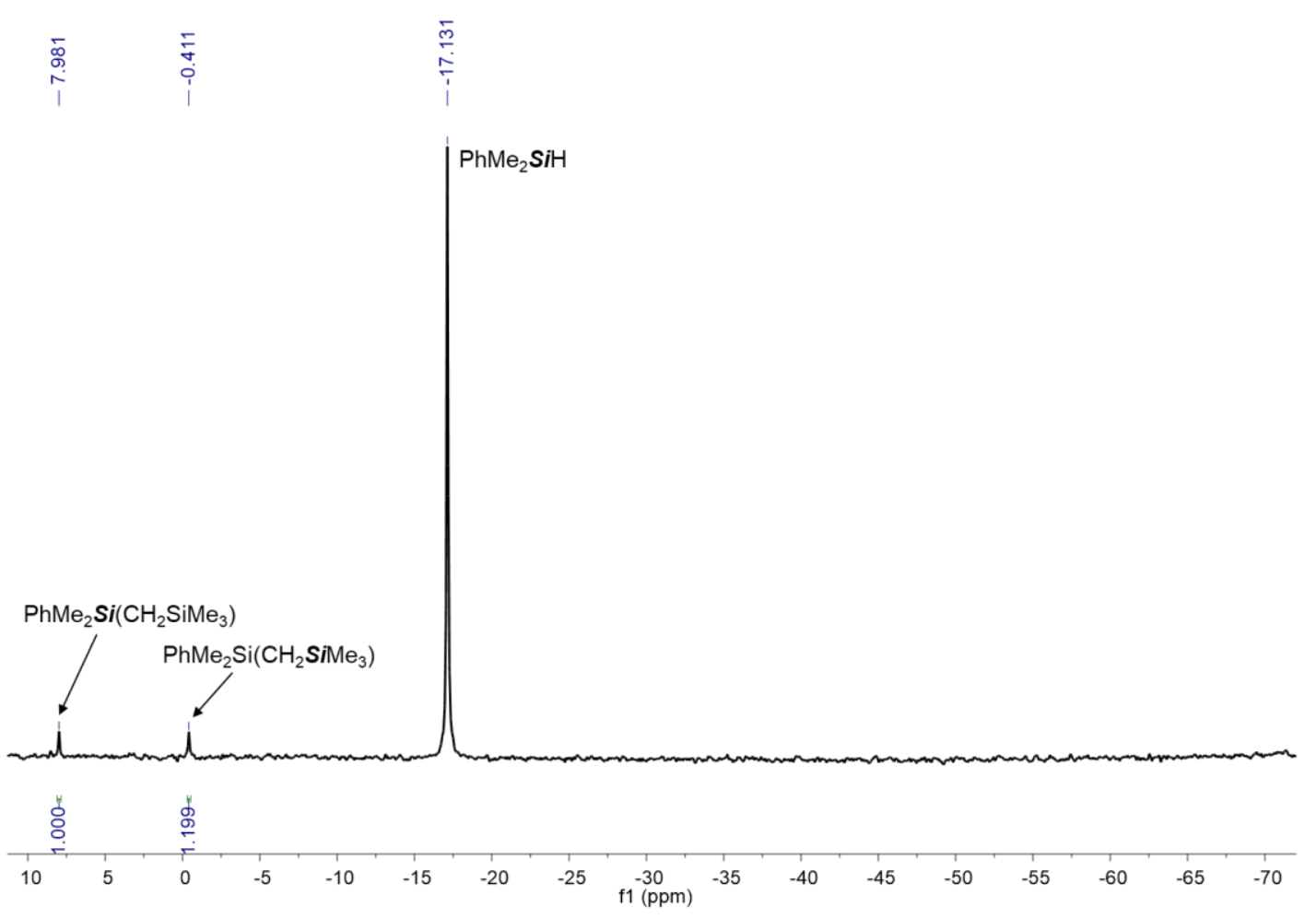

Figure $\mathbf{S 4 4}{ }^{29} \mathrm{Si}\left\{{ }^{1} \mathrm{H}\right\}$ NMR $\left(79.53 \mathrm{MHz}, \mathrm{C}_{6} \mathrm{D}_{6}\right)$ spectrum obtained from the reaction of complex 2 with five equivalents of $\mathrm{PhMe}_{2} \mathrm{SiH}$ and five equivalents of benzaldehyde.

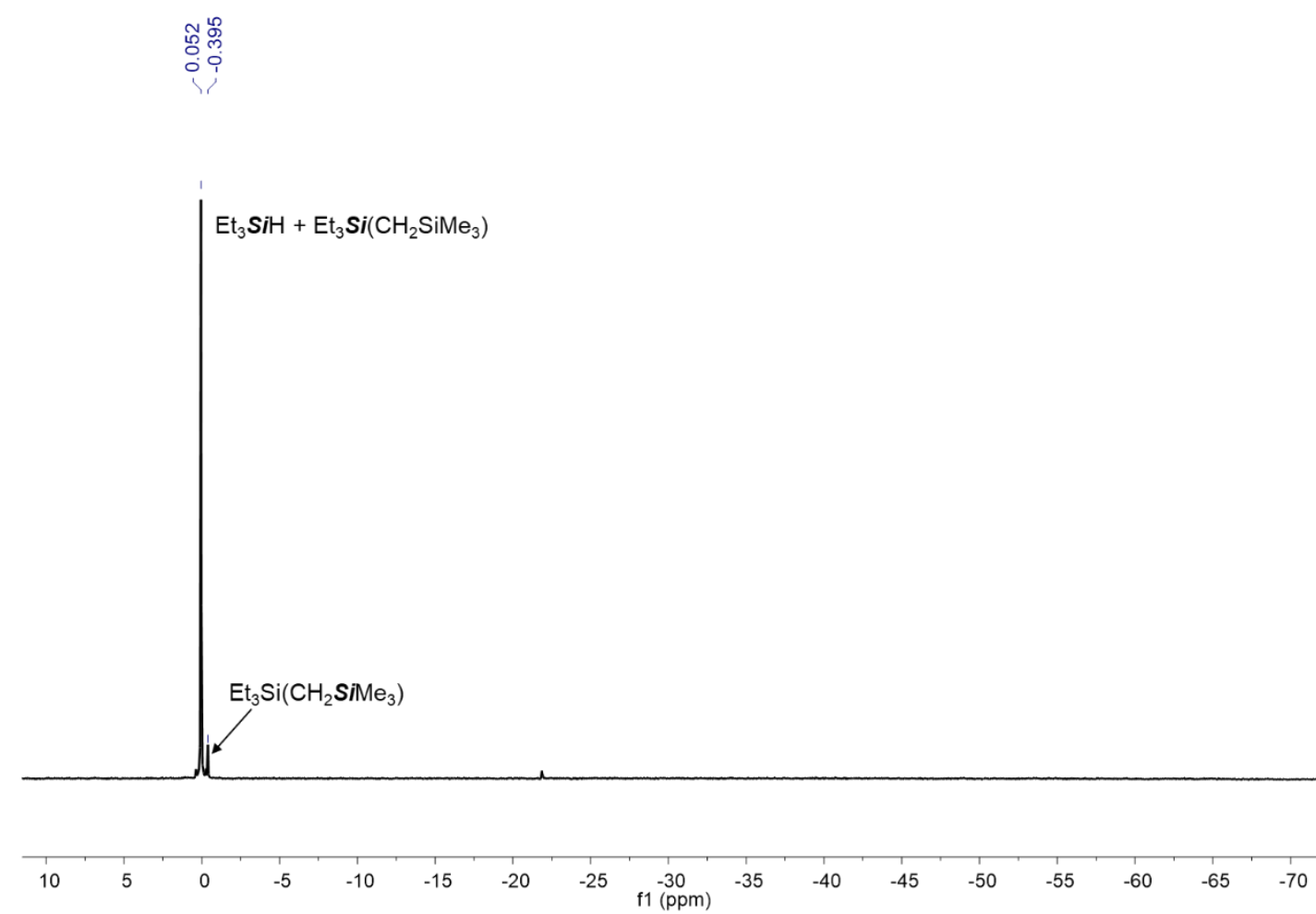

Figure $\mathbf{S 4 5}{ }^{29} \mathrm{Si}\left\{{ }^{1} \mathrm{H}\right\}$ NMR $\left(79.53 \mathrm{MHz}, \mathrm{C}_{6} \mathrm{D}_{6}\right)$ spectrum obtained from the reaction of complex 2 with five equivalents of $\mathrm{Et}_{3} \mathrm{SiH}$ and five equivalents of benzaldehyde. Note 1: the peak corresponding to the silicon $\mathrm{Et}_{3} \mathrm{Si}_{(}\left(\mathrm{CH}_{2} \mathrm{SiMe}_{3}\right)$ resonance is superimposed with that of $\mathrm{Et}_{3} \mathrm{SiH}$. Note 2: the unassigned resonance at $-22 \mathrm{ppm}$ corresponds to the silicon resonance of a residual amount of commercial silicone grease. 


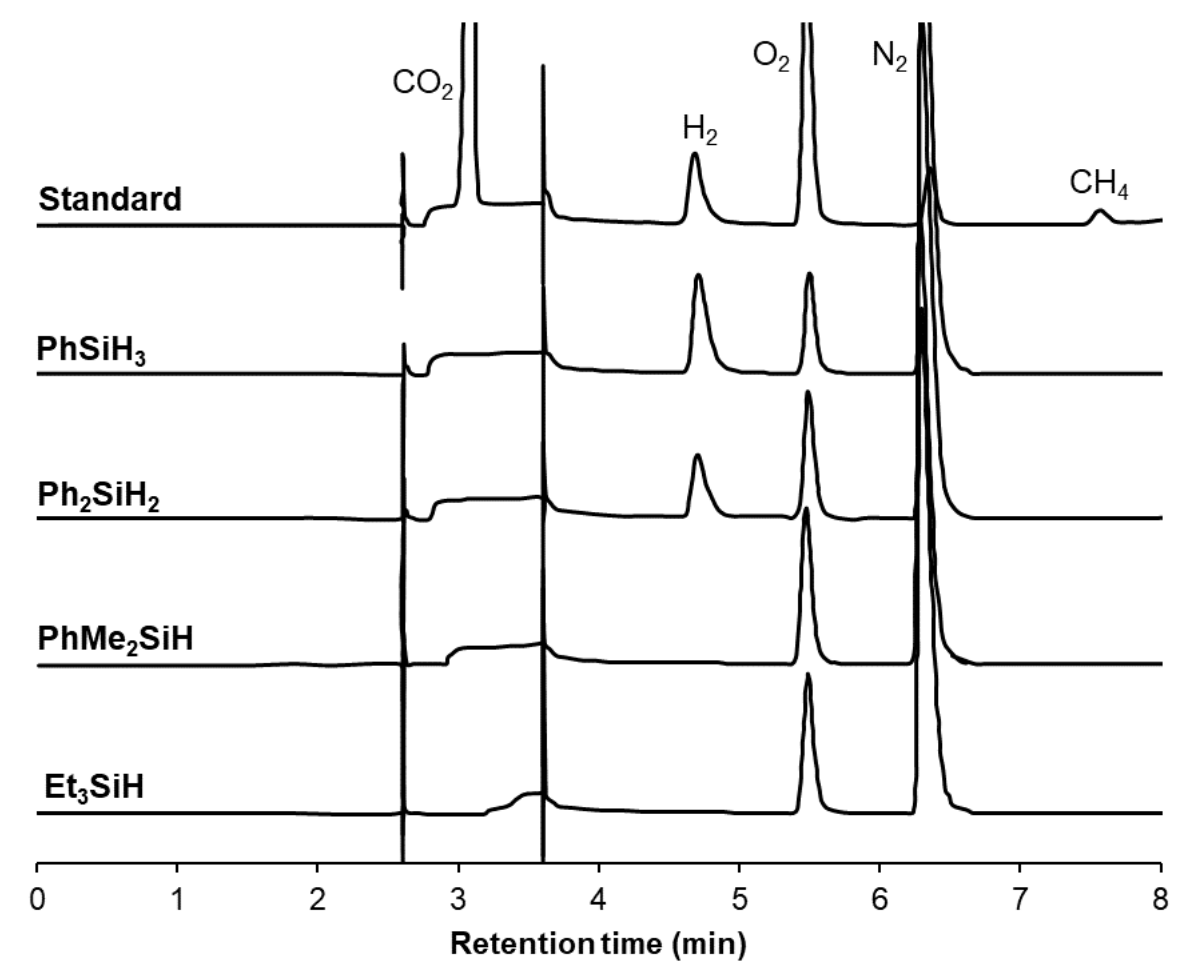

Figure S46 Stacking of the GC-TCD chromatograms evidencing the evolution of dihydrogen gas, or its absence, after the reaction of complex 2 with five equivalents of $\mathrm{R}_{3} \mathrm{SiH}\left(\mathrm{R}_{3}=\mathrm{PhH}_{2}\right.$ (second from top), $\mathrm{Ph}_{2} \mathrm{H}$ (third from top), $\mathrm{PhMe}_{2}$ (fourth from top), $\mathrm{Et}_{3}$ (fifth from top)) and five equivalents of benzaldehyde. The top chromatogram corresponds to a standard gas mixture containing dihydrogen, dioxygen, dinitrogen, methane and carbon dioxide. Note 1: the dioxygen detected in the samples was originated from the air-equilibrated needle upon injection. Note 2: the sharp peaks observed at retention times below 4 minutes correspond to the opening of the valves within the equipment used. 


\section{Supplementary kinetic data}

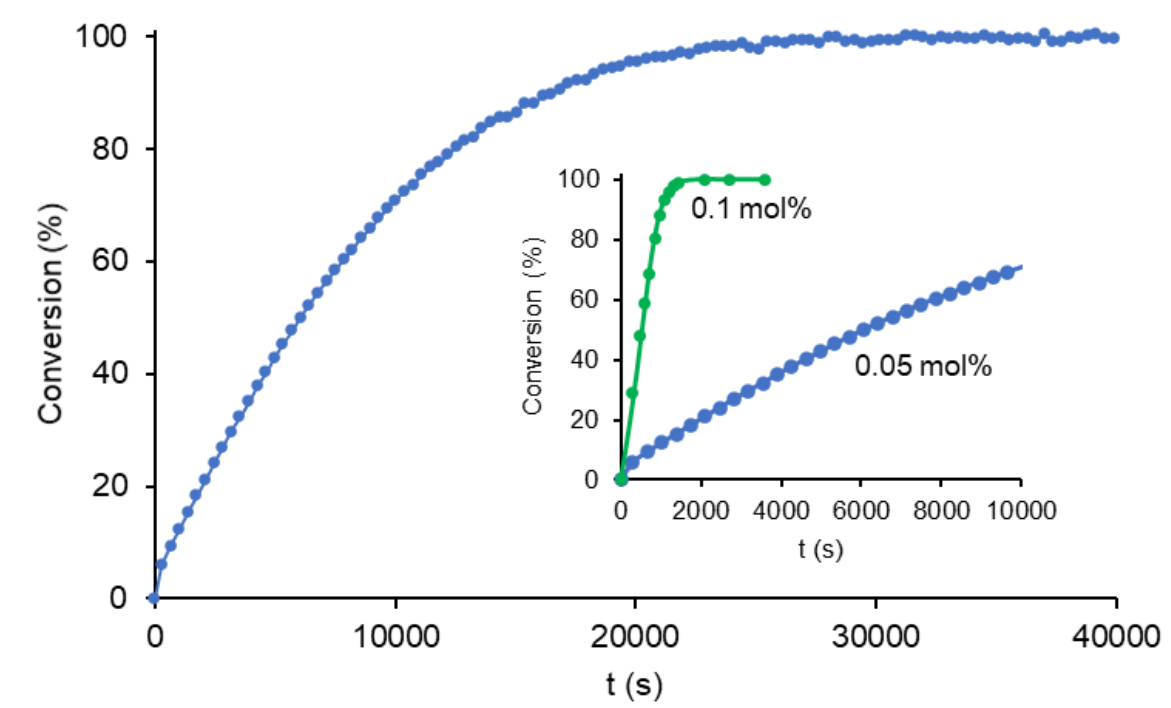

Figure S47 Kinetics of the hydrosilylation of benzaldehyde with $\mathrm{PhSiH}_{3}$ catalyzed by complex 2, at $25^{\circ} \mathrm{C}$ : Evolution of the conversion of benzaldehyde with time. The colored lines are a guide to the eye. Initial conditions: $0.05 \mathrm{~mol} \%$ of $2 ; 0.8 \mathrm{mmol}$ of benzaldehyde; $0.8 \mathrm{mmol}$ of $\mathrm{PhSiH}_{3}$; Solvent: $\mathrm{C}_{6} \mathrm{D}_{6}(0.5 \mathrm{~mL})$. The conversions of benzaldehyde were determined by ${ }^{1} \mathrm{H}$ NMR spectroscopy. The inset shows the kinetic runs of the hydrosilylation of benzaldehyde with $\mathrm{PhSiH}_{3}$ catalyzed by 0.1 mol\% (green data) or $0.05 \mathrm{~mol} \%$ (blue data) of complex 2 , at $25^{\circ} \mathrm{C}$, up until $10000 \mathrm{~s}$.

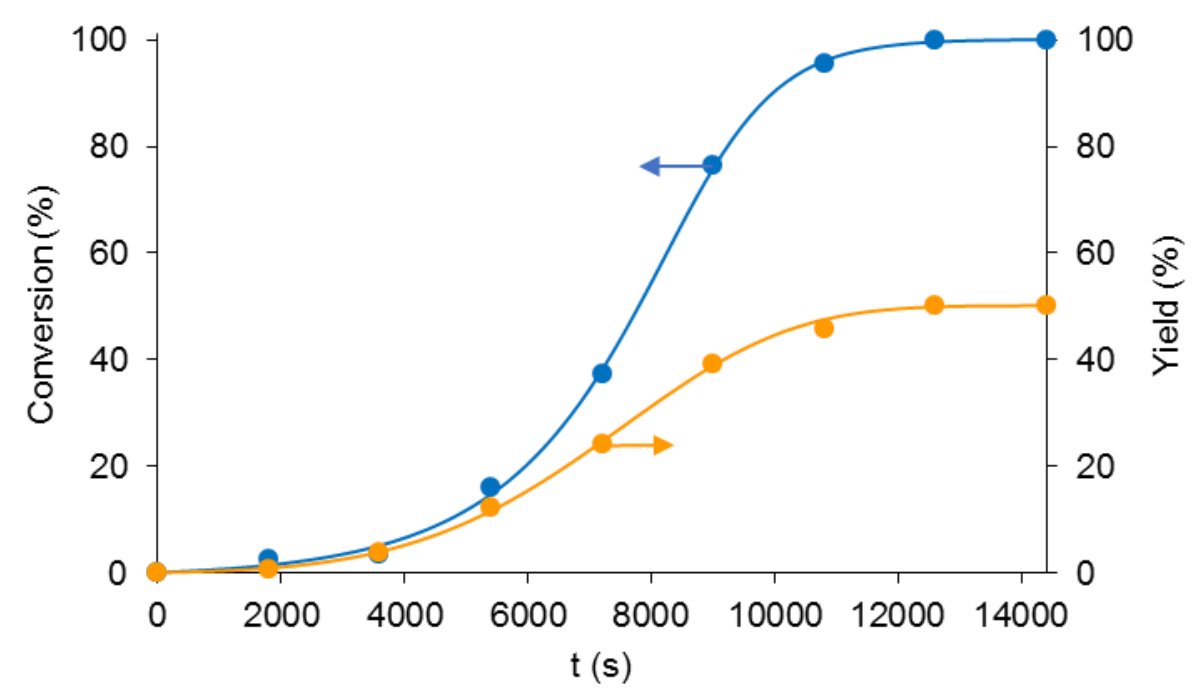

Figure S48 Kinetics of the hydrosilylation of benzophenone with $\mathrm{PhSiH}_{3}$ catalyzed by complex 2, at $25^{\circ} \mathrm{C}$ : Evolution of the conversion of benzaldehyde (blue data) and of the yield of the silane $\mathrm{PhSiH}\left(\mathrm{OCHPh}_{2}\right)_{2}$ (yellow data) with time (yields based on the initial amount of $\mathrm{PhSiH}_{3}$ ). The colored lines are a guide to the eye. Initial conditions: $1 \mathrm{~mol} \%$ of 2; $0.8 \mathrm{mmol}$ of benzophenone; $0.8 \mathrm{mmol}$ of $\mathrm{PhSiH}_{3}$; Solvent: $\mathrm{C}_{6} \mathrm{D}_{6}(0.5 \mathrm{~mL})$. The conversions of benzophenone and yields of the catalysis product were determined by ${ }^{1} \mathrm{H}$ NMR spectroscopy. 


\section{Computational mechanistic studies}

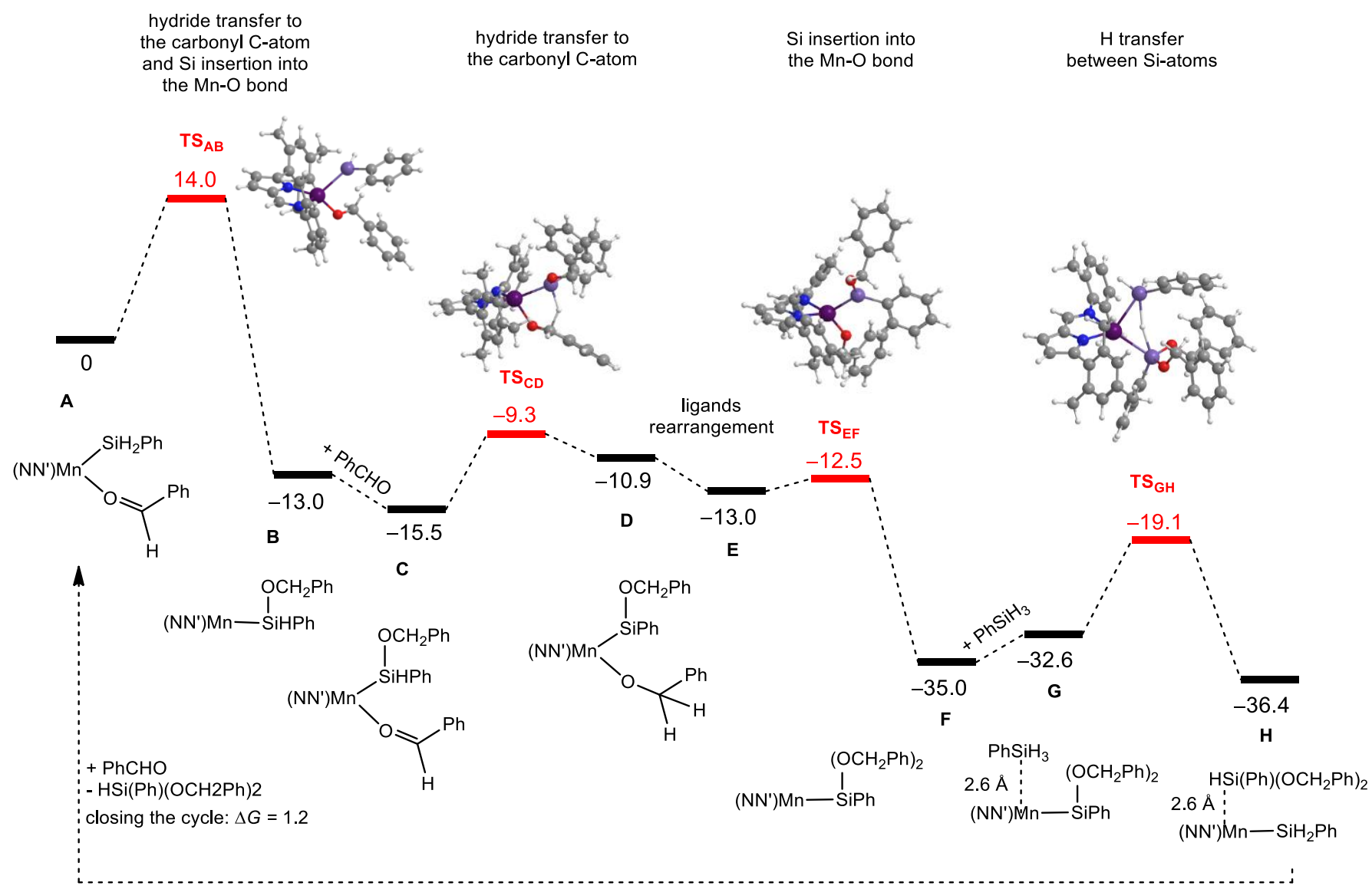

Figure $\mathbf{S 4 9}$ Gibbs energy profile $\left(\mathrm{kcal} \mathrm{mol}^{-1}\right)$ for the formation of $\mathrm{HSi}(\mathrm{Ph})\left(\mathrm{OCH}_{2} \mathrm{Ph}\right)_{2}(\mathrm{Cycle} \mathrm{I}$ in Scheme 4 of the article).

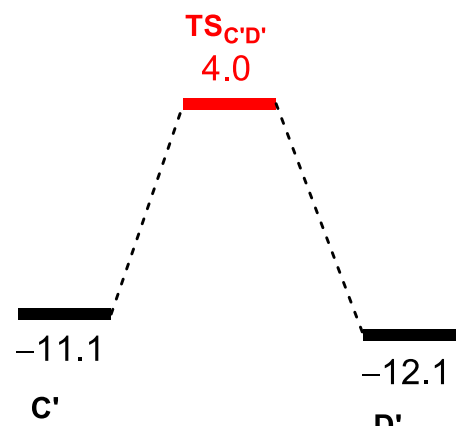

The barrier is ca.13 $\mathrm{kcal} / \mathrm{mol}$ higher than the one calculated for the reaction with another aldehyde $\left(\mathbf{T S}_{\mathrm{CD}}\right)$

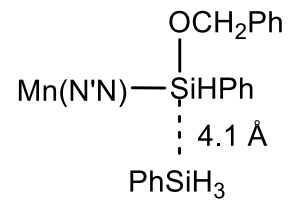

$\mathrm{H}_{2} \mathrm{Si}(\mathrm{Ph})\left(\mathrm{OCH}_{2} \mathrm{Ph}\right)$

$4.1 \AA$

$\mathrm{Mn}\left(\mathrm{N}^{\prime} \mathrm{N}\right)-\mathrm{SiH}_{2} \mathrm{Ph}$

Figure S50 Gibbs energy profile $\left(\mathrm{kcal} \mathrm{mol}^{-1}\right)$ for the addition of $\mathrm{PhSiH}_{3}$ as alternative to the second aldehyde. 


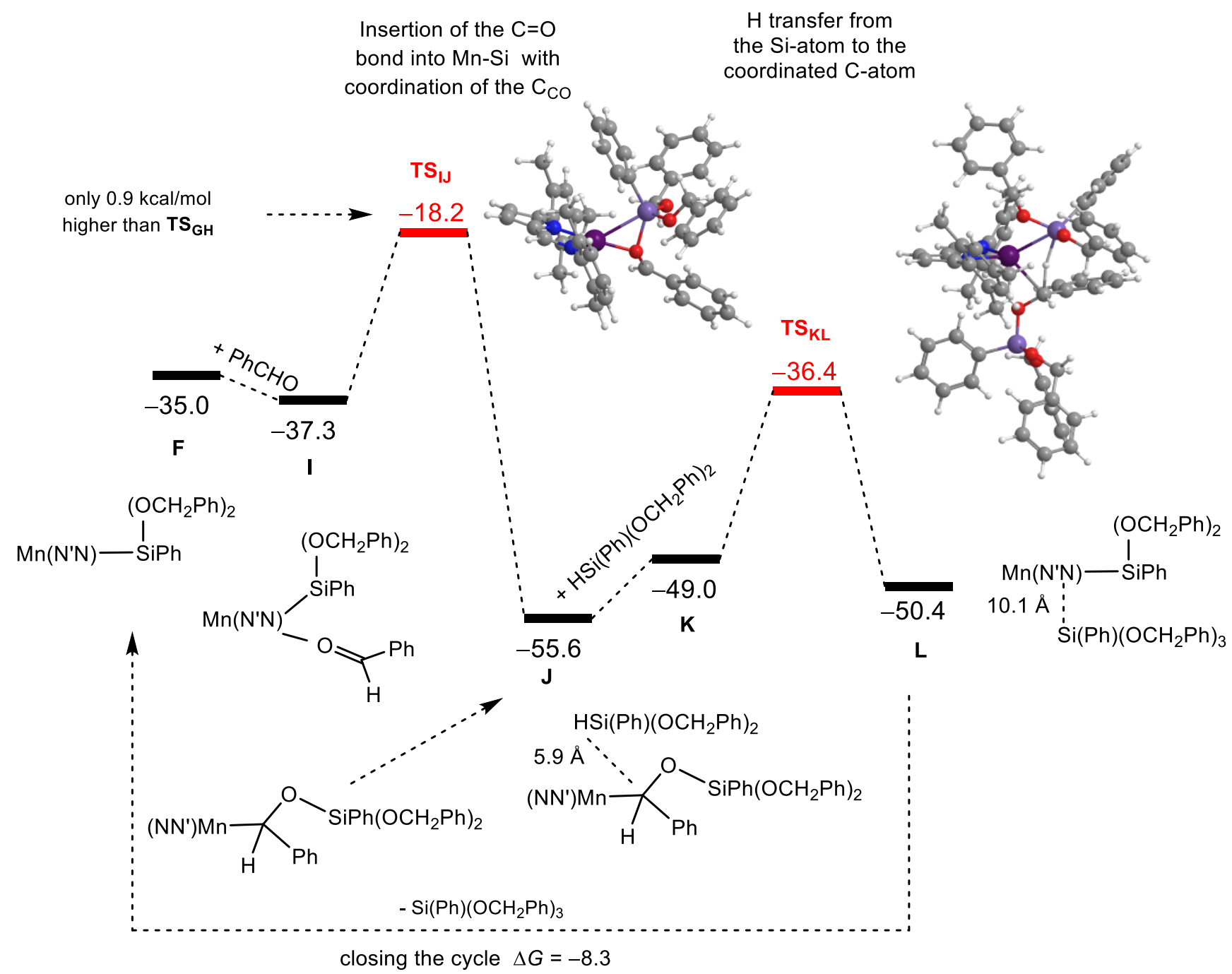

Figure S51 Gibbs energy profile $\left(\mathrm{kcal} \mathrm{mol}^{-1}\right)$ for the formation of $\mathrm{PhSi}\left(\mathrm{OCH}_{2} \mathrm{Ph}\right)_{3}$ from $\mathrm{HSi}(\mathrm{Ph})\left(\mathrm{OCH}_{2} \mathrm{Ph}\right)_{2}(\mathrm{Cycle}$ II in Scheme 34 of the article). 


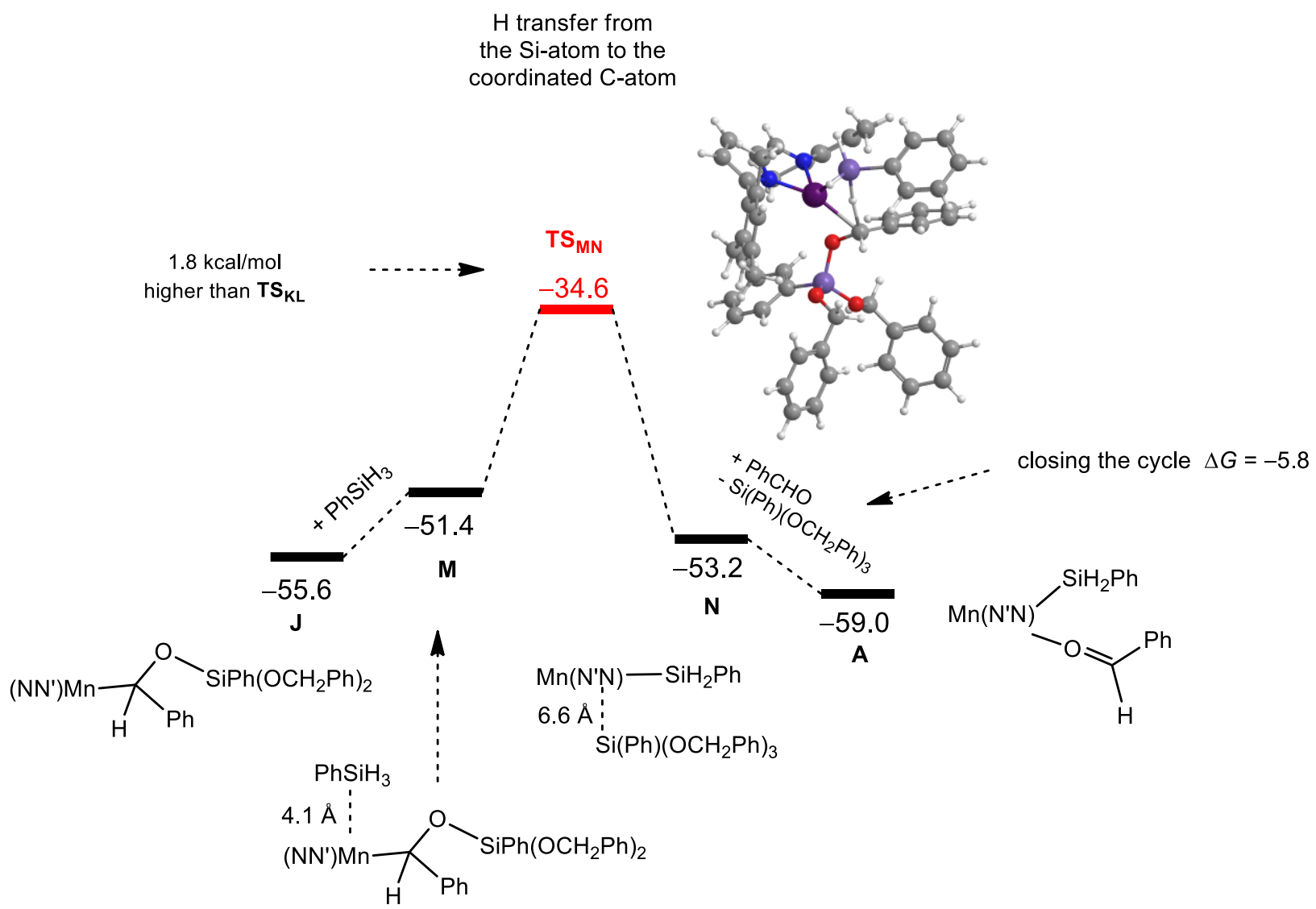

Figure S52 Gibbs energy profile ( $\left.\mathrm{kcal} \mathrm{mol}^{-1}\right)$ for the formation of $\mathrm{Si}(\mathrm{Ph})\left(\mathrm{OCH}_{2} \mathrm{Ph}\right)_{3}$ from $\mathrm{PhSiH}_{3}$.

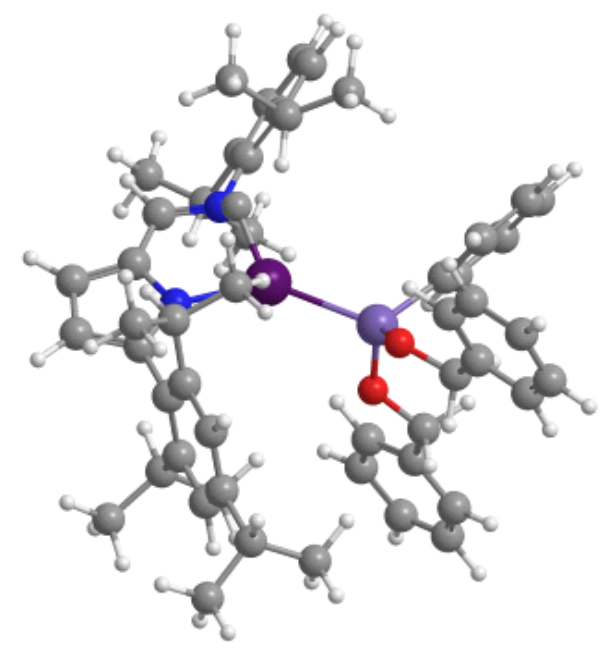

$F^{R}$

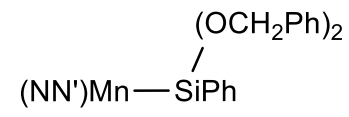
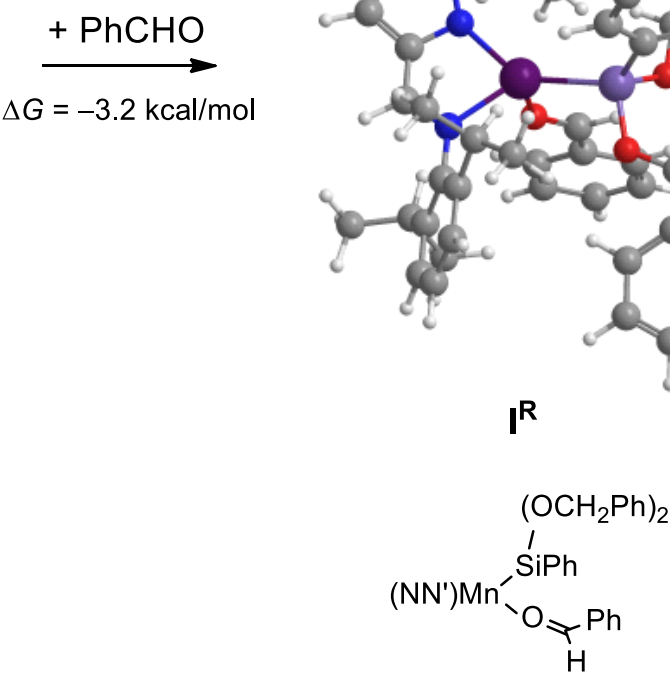

Figure S53 Optimized structures of intermediates $\mathbf{F}$ and $\mathbf{I}$ with the authentic ligands ( $\mathbf{F}^{\mathbf{R}}$ and $\mathbf{I}^{\mathbf{R}}$, respectively) and free energy balance (kcal/mol) of the corresponding step, $\mathbf{F}^{\mathbf{R}} \rightarrow \mathbf{I}^{\mathbf{R}}$. NN' denotes the chelating ligand $\left[\kappa^{2} N, N^{\prime}-5-\left(2,4,6-i \operatorname{Pr}_{3} \mathrm{C}_{6} \mathrm{H}_{2}\right)-\mathrm{NC}_{4} \mathrm{H}_{2}-2-\mathrm{C}(\mathrm{H})=\mathrm{N}\left(2,6-i \operatorname{Pr}_{2} \mathrm{C}_{6} \mathrm{H}_{3}\right)\right]$. 


\section{Atomic coordinates of the optimized geometries}

\section{(NN')Mn(Py)(CH2SiMe3)}

\begin{tabular}{|c|c|c|c|}
\hline $\mathrm{Mn}$ & 0.351989 & -0.308976 & -0.594834 \\
\hline $\mathrm{Si}$ & 1.090378 & -3.032286 & 1.510910 \\
\hline $\mathrm{N}$ & -1.421339 & 0.857149 & -0.991854 \\
\hline $\mathrm{N}$ & 0.845269 & 0.563403 & -2.606092 \\
\hline N & 1.512906 & 1.196699 & 0.660608 \\
\hline C & -3.142651 & 0.702929 & 0.831042 \\
\hline C & -1.313276 & 1.514501 & -2.220725 \\
\hline C & -2.474777 & 2.290792 & -2.469444 \\
\hline $\mathrm{H}$ & -2.666984 & 2.898307 & -3.355915 \\
\hline C & -3.308871 & 2.108428 & -1.356669 \\
\hline $\mathrm{H}$ & -4.298452 & 2.537862 & -1.193259 \\
\hline C & -2.635119 & 1.218477 & -0.468822 \\
\hline C & -0.142830 & 1.342990 & -3.011162 \\
\hline $\mathrm{H}$ & -0.080510 & 1.867676 & -3.978912 \\
\hline C & -3.397982 & -0.690732 & 0.999512 \\
\hline C & -3.900691 & -1.154202 & 2.228285 \\
\hline $\mathrm{H}$ & -4.104877 & -2.226152 & 2.342848 \\
\hline C & -4.162812 & -0.284851 & 3.305992 \\
\hline C & -3.919344 & 1.085440 & 3.116737 \\
\hline $\mathrm{H}$ & -4.121681 & 1.782958 & 3.939540 \\
\hline $\mathrm{C}$ & -3.424170 & 1.598115 & 1.900435 \\
\hline C & -3.219414 & 3.094964 & 1.770425 \\
\hline $\mathrm{H}$ & -2.352232 & 3.345836 & 1.137817 \\
\hline $\mathrm{H}$ & -3.077702 & 3.558446 & 2.761503 \\
\hline $\mathrm{H}$ & -4.095465 & 3.585276 & 1.306717 \\
\hline $\mathrm{H}$ & 1.659383 & -2.789446 & -4.555521 \\
\hline $\mathrm{H}$ & 0.337305 & -1.591933 & -4.565886 \\
\hline $\mathrm{H}$ & 1.984785 & 3.468991 & -3.319389 \\
\hline $\mathrm{H}$ & 3.625671 & 3.419741 & -2.641558 \\
\hline $\mathrm{H}$ & -3.638762 & -2.647313 & 0.100603 \\
\hline C & -3.173954 & -1.674692 & -0.129860 \\
\hline $\mathrm{H}$ & -3.597759 & -1.303152 & -1.079096 \\
\hline $\mathrm{H}$ & -2.097720 & -1.852082 & -0.312804 \\
\hline $\mathrm{H}$ & -4.888736 & -0.002905 & 5.336699 \\
\hline $\mathrm{H}$ & -3.980566 & -1.517340 & 5.089708 \\
\hline C & -4.694696 & -0.817127 & 4.619183 \\
\hline $\mathrm{H}$ & -5.638473 & -1.374065 & 4.476655 \\
\hline C & 2.030638 & 0.449181 & -3.390735 \\
\hline C & 2.964923 & 1.521246 & -3.444492 \\
\hline C & 4.163316 & 1.332486 & -4.161976 \\
\hline $\mathrm{H}$ & 4.889831 & 2.152893 & -4.199142 \\
\hline $\mathrm{C}$ & 4.436355 & 0.124924 & -4.819021 \\
\hline $\mathrm{H}$ & 5.375023 & -0.003579 & -5.368413 \\
\hline C & 3.499349 & -0.917795 & -4.769315 \\
\hline $\mathrm{H}$ & 3.699794 & -1.861116 & -5.290750 \\
\hline C & 2.290271 & -0.777967 & -4.062257 \\
\hline $\mathrm{C}$ & 1.268185 & -1.891365 & -4.050122 \\
\hline $\mathrm{H}$ & 0.980368 & -2.170703 & -3.021077 \\
\hline C & 2.694346 & 2.839807 & -2.751239 \\
\hline $\mathrm{H}$ & 2.255044 & 2.694162 & -1.750324 \\
\hline C & 1.004624 & -2.270799 & -0.205874 \\
\hline $\mathrm{H}$ & 0.412723 & -2.969212 & -0.837436 \\
\hline $\mathrm{H}$ & 2.034095 & -2.294346 & -0.632138 \\
\hline C & 1.940136 & -1.888620 & 2.782123 \\
\hline $\mathrm{H}$ & 2.968973 & -1.620952 & 2.480250 \\
\hline $\mathrm{H}$ & 2.010616 & -2.382456 & 3.769049 \\
\hline $\mathrm{H}$ & 1.376200 & -0.949466 & 2.924439 \\
\hline C & 2.081175 & -4.670276 & 1.504002 \\
\hline $\mathrm{H}$ & 1.627543 & -5.411658 & 0.820860 \\
\hline $\mathrm{H}$ & 2.127022 & -5.130550 & 2.508731 \\
\hline $\mathrm{H}$ & 3.121923 & -4.510599 & 1.166516 \\
\hline C & -0.646793 & -3.437230 & 2.183347 \\
\hline $\mathrm{H}$ & -1.261166 & -2.529898 & 2.321658 \\
\hline $\mathrm{H}$ & -0.580635 & -3.942228 & 3.164864 \\
\hline $\mathrm{H}$ & -1.198191 & -4.113431 & 1.505103 \\
\hline C & 0.877133 & 2.158734 & 1.372405 \\
\hline $\mathrm{H}$ & -0.211172 & 2.195315 & 1.257645 \\
\hline C & 1.556695 & 3.057585 & 2.203980 \\
\hline $\mathrm{H}$ & 0.995525 & 3.814815 & 2.758733 \\
\hline $\mathrm{C}$ & 2.952951 & 2.964067 & 2.303175 \\
\hline $\mathrm{H}$ & 3.513526 & 3.651990 & 2.94407 \\
\hline
\end{tabular}

$\begin{array}{llll}\mathrm{C} & 3.617911 & 1.972336 & 1.566002 \\ \mathrm{H} & 4.705017 & 1.861525 & 1.610536 \\ \mathrm{C} & 2.862400 & 1.110337 & 0.762005 \\ \mathrm{H} & 3.338390 & 0.318914 & 0.173699\end{array}$

\section{PhCHO}

$\begin{array}{cccc}\text { C } & 3.576553 & 1.557491 & 2.719507 \\ \mathrm{H} & 5.005798 & 0.843531 & 1.263904 \\ \mathrm{O} & 2.498254 & 0.157964 & 1.084343 \\ \mathrm{C} & 4.871024 & 1.451831 & 2.163971 \\ \mathrm{H} & 6.948520 & 2.037527 & 2.338913 \\ \mathrm{C} & 5.943472 & 2.116768 & 2.765793 \\ \mathrm{H} & 6.573322 & 3.409846 & 4.392738 \\ \mathrm{C} & 5.731158 & 2.890252 & 3.923658 \\ \mathrm{H} & 4.286694 & 3.601833 & 5.380771 \\ \mathrm{C} & 4.445589 & 2.999250 & 4.480795 \\ \mathrm{H} & 2.360063 & 2.408633 & 4.302033 \\ \mathrm{C} & 3.369064 & 2.333498 & 3.879103 \\ \mathrm{C} & 2.429476 & 0.857898 & 2.094002 \\ \mathrm{H} & 1.449414 & 1.014377 & 2.620468\end{array}$

\section{$\mathrm{PhSiH}_{3}$}

$\begin{array}{rrrr}\text { H } & 1.610665 & -1.172198 & -0.457105 \\ \mathrm{C} & 3.506627 & -3.192852 & -0.003928 \\ \mathrm{Si} & 1.852367 & -2.378006 & 0.401896 \\ \mathrm{C} & 4.479886 & -3.393645 & 1.002570 \\ \mathrm{H} & 0.703326 & -3.317907 & 0.183466 \\ \mathrm{C} & 5.710485 & -4.003753 & 0.707826 \\ \mathrm{H} & 1.850967 & -1.952690 & 1.838393 \\ \mathrm{C} & 5.990302 & -4.423596 & -0.601825 \\ \mathrm{H} & 4.277065 & -3.069638 & 2.029887 \\ \mathrm{C} & 5.036482 & -4.231961 & -1.615874 \\ \mathrm{H} & 6.450726 & -4.149450 & 1.501839 \\ \mathrm{C} & 3.808200 & -3.622476 & -1.318836 \\ \mathrm{H} & 6.949402 & -4.898956 & -0.833479 \\ \mathrm{H} & 5.250862 & -4.557192 & -2.639558 \\ \mathrm{H} & 3.077540 & -3.480781 & -2.124370\end{array}$

\section{$\mathrm{SiMe}_{4}$}

Si $\quad 3.269352$

$-2.760982$

1.980403

0.319074

0.268392

$-0.161504$

$-0.444118$

2. 662969

1. 997152

3. 657205

2.770190

1. 829858

1. 441226

2.808290

1. 144062

3. 161756

3. 276456

4.168423

2. 794466

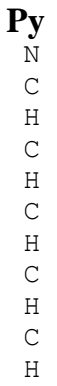

7.371338

6.854079

5.903091

7.460300

6.988787

8.674984

9.179951

9.228605

10.174023

8.540837

8.948106 $\begin{array}{ll}4.152465 & 15.067940 \\ 5.395452 & 15.169080 \\ 5.563951 & 14.646918 \\ 6.438934 & 15.886415 \\ 7.425710 & 15.929289 \\ 6.183236 & 16.539633 \\ 6.970004 & 17.109839 \\ 4.897811 & 16.446684 \\ 4.649390 & 16.939024 \\ 3.925551 & 15.703297 \\ 2.910098 & 15.611682\end{array}$ 


\section{$\mathrm{PhSiH}_{2} \mathrm{CH}_{2} \mathrm{SiMe}_{3}$}

$\begin{array}{rr}\text { Si } & -1.044636 \\ \mathrm{H} & -1.562278 \\ \mathrm{C} & -1.228586 \\ \mathrm{H} & -2.049404 \\ \mathrm{C} & 0.342501 \\ \mathrm{H} & 1.333794 \\ \mathrm{H} & 0.388419 \\ \mathrm{H} & 0.184011 \\ \mathrm{C} & -0.650646 \\ \mathrm{H} & -1.426794 \\ \mathrm{H} & -0.591796 \\ \mathrm{H} & 0.315482 \\ \mathrm{C} & -2.686010 \\ \mathrm{H} & -2.941772 \\ \mathrm{H} & -2.635806 \\ \mathrm{H} & -3.524051 \\ \mathrm{H} & 0.795205 \\ \mathrm{C} & -0.050730 \\ \mathrm{Si} & 0.292346 \\ \mathrm{C} & 0.254902 \\ \mathrm{H} & 1.393277 \\ \mathrm{C} & -0.032800 \\ \mathrm{C} & -0.632353 \\ \mathrm{H} & 0.729848 \\ \mathrm{C} & -0.939911 \\ \mathrm{H} & 0.214994 \\ \mathrm{C} & -0.650099 \\ \mathrm{H} & -0.855500 \\ \mathrm{H} & -1.401908 \\ \mathrm{H} & -0.891001 \\ & \end{array}$

\section{$\mathrm{PhSiH}\left(\mathrm{OCH}_{2} \mathrm{Ph}\right)_{2}$}

$\begin{array}{rrrr}\mathrm{C} & 5.691743 & -0.260219 & 0.932775 \\ \mathrm{H} & 4.808566 & 1.118707 & -0.474758 \\ \mathrm{O} & 3.259578 & -0.119801 & 0.860373 \\ \mathrm{C} & 5.744054 & 0.708537 & -0.084421 \\ \mathrm{H} & 7.008243 & 1.898283 & -1.381653 \\ \mathrm{C} & 6.982112 & 1.142598 & -0.588976 \\ \mathrm{H} & 9.142940 & 0.952919 & -0.476597 \\ \mathrm{C} & 8.179032 & 0.613931 & -0.082774 \\ \mathrm{H} & 9.060684 & -0.775741 & 1.336435 \\ \mathrm{C} & 8.132459 & -0.355406 & 0.934641 \\ \mathrm{H} & 6.868085 & -1.545868 & 2.231043 \\ \mathrm{C} & 6.897780 & -0.788808 & 1.437772 \\ \mathrm{C} & 4.374995 & -0.750100 & 1.509387 \\ \mathrm{H} & 4.342681 & -0.529981 & 2.595394 \\ \mathrm{C} & 1.628623 & -2.506376 & 0.359577 \\ \mathrm{Si} & 1.699534 & -0.729644 & 0.952876 \\ \mathrm{C} & 0.740586 & -3.445699 & 0.934916 \\ \mathrm{H} & 1.602161 & 1.026665 & 3.440377 \\ \mathrm{C} & 0.666106 & -4.759308 & 0.445776 \\ \mathrm{H} & 4.313783 & -1.852688 & 1.405969 \\ \mathrm{C} & 1.478761 & -5.156670 & -0.628939 \\ \mathrm{H} & 0.107270 & -3.144055 & 1.776026 \\ \mathrm{C} & 2.367599 & -4.238691 & -1.212362 \\ \mathrm{H} & -0.025856 & -5.474215 & 0.903863 \\ \mathrm{C} & 2.440781 & -2.925560 & -0.721890 \\ \mathrm{H} & 1.420041 & -6.181403 & -1.011235 \\ \mathrm{H} & 3.004802 & -4.546230 & -2.048396 \\ \mathrm{H} & 3.142718 & -2.220301 & -1.182447 \\ \mathrm{C} & -0.084844 & 0.142196 & 4.451605 \\ \mathrm{H} & 0.721532 & -1.850208 & 4.687404 \\ \mathrm{O} & 1.058710 & -0.758415 & 2.501324 \\ \mathrm{C} & 0.066705 & -1.087586 & 5.118890 \\ \mathrm{H} & -0.499449 & -2.298266 & 6.824075 \\ \mathrm{C} & -0.622473 & -1.335039 & 6.317124 \\ \mathrm{H} & -2.002789 & -0.549674 & 7.799524 \\ \mathrm{C} & -1.465343 & -0.355019 & 6.865487 \\ \mathrm{H} & -2.280865 & 1.643353 & 6.620041 \\ \mathrm{C} & -1.619645 & 0.875229 & 6.204873 \\ \mathrm{H} & -1.068204 & 2.077632 & 4.486702 \\ \mathrm{C} & -0.936718 & 1.119447 & 5.003695 \\ \mathrm{H} & 0.687744 & 0.453001 & 3.183609 \\ \mathrm{H} & 0.077530 & 1.101130 & 2.522821 \\ \mathrm{H} & & 0.247143 & 0.117274\end{array}$

$$
\begin{array}{rr}
-1.999786 & 4.845634 \\
-0.167386 & 3.234023 \\
-1.216920 & 3.114222 \\
-1.739145 & 2.583918 \\
-1.136572 & 5.814458 \\
-1.307569 & 5.359177 \\
-1.510761 & 6.853112 \\
-0.044448 & 5.864067 \\
-3.849608 & 4.676977 \\
-4.378858 & 4.095715 \\
-4.334650 & 5.667890 \\
-4.016427 & 4.169419 \\
-1.784140 & 5.780087 \\
-0.716702 & 5.905177 \\
-2.232367 & 6.788902 \\
-2.265127 & 5.244607 \\
-2.626572 & 1.815708 \\
-0.501052 & 0.272363 \\
-1.220784 & 1.990796 \\
0.847460 & -0.029731 \\
-0.407286 & 2.609995 \\
1.393798 & -1.291067 \\
0.598284 & -2.280763 \\
1.481550 & 0.728766 \\
-0.744357 & -2.004556 \\
2.439682 & -1.502798 \\
-1.285837 & -0.742363 \\
1.021597 & -3.265999 \\
-1.371139 & -2.775175 \\
-2.338478 & -0.548858
\end{array}
$$

$$
\begin{array}{r}
0.932775 \\
-0.474758 \\
0.860373 \\
-0.084421 \\
-1.381653 \\
-0.588976 \\
-0.476597 \\
-0.082774 \\
1.336435 \\
0.934641 \\
2.231043 \\
1.437772 \\
1.509387 \\
2.595394 \\
0.359577 \\
0.952876 \\
0.934916 \\
3.440377 \\
0.445776 \\
1.405969 \\
-0.628939 \\
1.776026 \\
-1.212362 \\
0.903863 \\
-0.721890 \\
-1.011235 \\
-2.048396 \\
-1.182447 \\
4.451605 \\
4.687404 \\
2.501324 \\
5.118890 \\
6.824075 \\
6.317124 \\
7.799524 \\
6.865487 \\
6.620041 \\
6.204873 \\
4.486702 \\
5.003695 \\
3.183609 \\
2.522821 \\
0.117274
\end{array}
$$

\section{$\mathbf{P h S i}\left(\mathrm{OCH}_{2} \mathbf{P h}\right)_{3}$}

6.036723
6.680778

3.810289

6.772476

8.188522

7.618712

8.403233

7. 739554

7.105138

7.011103

5.597167

6.164220

5.102013

4. 984225

2.000850

2.495631

2.594293

2. 446617

2.238449

5.514338

1. 278106

3.332905

0.673772

2. 707037

1.033187

0.998222

$-0.081300$

0.549047

0.821576

$-1.214264$

1.258988

$-0.556416$

$-2.158994$

$-1.087322$

$-0.655123$

$-0.243054$

1. 793989

1.131383

2. 730767

1.659516

1. 388634

0.826746

4. 371716

4.954752

2. 787773

5. 287536

7. 312831

6.611423

8.069660

7.036818

6.455114

6.130920

4. 106800

4.808295

2. 925866

2. 436987

2. 371777

3.476772

4. 121359

3. 007385

4.287260

5.914985

5.294755

6.286591

5. 503369

4.856480

4.700897

3.066426

3. 694719

2. 412344

1.603389

0.752445

2. 102077

$-0.532662$

4. 940968

$-1.520518$

1.969346

$-1.242872$

$-0.769087$

0.023900

$-2.509522$

1. 011224

$-2.014269$

0.242238

1. 992612

5.199813

5. 300342

3. 201267

5. 472878

6.178677

5. 966146

6.583681

6.193514

6.102838

5.923636

5.214769

5. 428206

4. 645676

5.045784

2. 122061

0.151267

1. 301332

1.065338

0.355500

1. 183248

2. 447719

2. 356846

4. 333246

3.412936

4. 127470

3.296670

1. 979104

2. 971541

1. 368186

2.258555

0.291338

1. 444487

1.370646

1. 157997

1. 858125

3. 623880

3. 242435

5. 215925

4. 135891

4. 341462

3. 644748

1. 727899

2. 474353

2. 127265

0.931384

2. 088715

0.716687

3. 020164

0.801804

4. 007037

1. 315047

4.057457

2. 973122

3.126786

4. 732609

4.820319

3.167841

2. 113141

1. 371906

0.764096

2. 231730

3. 510742

3. 433446

5.470173

4.533453

5. 280001

4.426533

3. 138761

3.223977

0.822364

$-0.040915$

$-2.213836$

$-1.528664$

$-0.509105$

$-2.031212$

$-2.330735$

$-2.481156$

$-3.479469$

$-3.127223$

$-3.813484$

$-3.315948$

$-2.997190$

$-2.856502$

$-1.772938$

$-1.749158$

$-2.511867$

\section{(NN')Mn(PhCHO)(H)}

$\begin{array}{rrr}\text { Mn } & 0.193142 & -1.168832 \\ \mathrm{~N} & -1.600762 & -0.215946 \\ \mathrm{~N} & 0.686659 & -0.543367 \\ \mathrm{C} & -3.261809 & -0.278411 \\ \mathrm{C} & -1.531544 & 0.301485 \\ \mathrm{C} & -2.743749 & 0.965926 \\ \mathrm{H} & -2.979729 & 1.458009 \\ \mathrm{C} & -3.560719 & 0.858187 \\ \mathrm{H} & -4.576051 & 1.238812 \\ \mathrm{C} & -2.828238 & 0.121131 \\ \mathrm{C} & -0.334322 & 0.113272 \\ \mathrm{H} & -0.273977 & 0.504066 \\ \mathrm{C} & -3.340518 & -1.656903 \\ \mathrm{C} & -3.752113 & -2.008772 \\ \mathrm{H} & -3.820220 & -3.072926\end{array}$

$-0.489631$

$-1.041791$

$-2.546698$

0.821333

$-2.336186$

$-2.658022$

$-3.603387$

$-1.521851$

$-1.402208$

$-0.543571$

$-3.079382$

$-4.107307$

1.176859

2.475577

2. 735049 


\begin{tabular}{|c|c|}
\hline $\mathrm{C}$ & -4.090094 \\
\hline $\mathrm{C}$ & -4.019155 \\
\hline $\mathrm{H}$ & -4.281640 \\
\hline C & -3.615518 \\
\hline $\mathrm{C}$ & -3.560284 \\
\hline $\mathrm{H}$ & -2.640930 \\
\hline $\mathrm{H}$ & -3.601150 \\
\hline $\mathrm{H}$ & -4.408428 \\
\hline $\mathrm{H}$ & 2.048941 \\
\hline $\mathrm{H}$ & 0.552118 \\
\hline $\mathrm{H}$ & 1.518462 \\
\hline $\mathrm{H}$ & 3.125452 \\
\hline $\mathrm{H}$ & -3.444692 \\
\hline $\mathrm{C}$ & -3.022668 \\
\hline $\mathrm{H}$ & -1.930299 \\
\hline $\mathrm{H}$ & -3.424046 \\
\hline $\mathrm{H}$ & -5.098418 \\
\hline $\mathrm{H}$ & -5.082497 \\
\hline C & -4.495546 \\
\hline $\mathrm{H}$ & -3.609396 \\
\hline C & 1.905588 \\
\hline C & 2.684165 \\
\hline $\mathrm{C}$ & 3.905458 \\
\hline $\mathrm{H}$ & 4.512807 \\
\hline C & 4.355448 \\
\hline $\mathrm{H}$ & 5.308066 \\
\hline C & 3.583552 \\
\hline $\mathrm{H}$ & 3.928602 \\
\hline C & 2.360889 \\
\hline C & 1.526617 \\
\hline $\mathrm{H}$ & 1.303013 \\
\hline $\mathrm{C}$ & 2.255940 \\
\hline $\mathrm{H}$ & 1.793846 \\
\hline $\mathrm{C}$ & 2.811952 \\
\hline $\mathrm{H}$ & 2.056282 \\
\hline 0 & 1.299223 \\
\hline C & 2. 712221 \\
\hline $\mathrm{H}$ & 3.376078 \\
\hline $\mathrm{C}$ & 3.448305 \\
\hline $\mathrm{H}$ & 4.860478 \\
\hline C & 4.285422 \\
\hline $\mathrm{H}$ & 5.040331 \\
\hline C & 4.388453 \\
\hline $\mathrm{H}$ & 3.720010 \\
\hline C & 3.652504 \\
\hline C & 2.061461 \\
\hline $\mathrm{H}$ & 2.192118 \\
\hline $\mathrm{H}$ & 0.718610 \\
\hline
\end{tabular}

\begin{tabular}{|c|c|}
\hline-1.042766 & 3.441420 \\
\hline 0.311102 & 3.067993 \\
\hline 1.083193 & 3.802455 \\
\hline 0.713210 & 1.780435 \\
\hline 2.193453 & 1.457877 \\
\hline 2.463067 & 0.911173 \\
\hline 2.793711 & 2.382152 \\
\hline 2.509433 & 0.824134 \\
\hline-4.161950 & -3.304532 \\
\hline-3.237240 & -3.608163 \\
\hline 2.225673 & -4.095889 \\
\hline 2.521130 & -3.404676 \\
\hline-2.542344 & -0.812904 \\
\hline-2.756285 & 0.183817 \\
\hline-2.881995 & 0.049924 \\
\hline-3.722797 & 0.531600 \\
\hline-2.374046 & 4.837297 \\
\hline-0.659982 & 5.339959 \\
\hline-1.449651 & 4.841660 \\
\hline-1.647109 & 5.474162 \\
\hline-0.702071 & -3.263571 \\
\hline 0.421371 & -3.663347 \\
\hline 0.185760 & -4.326975 \\
\hline 1.046933 & -4.629830 \\
\hline-1.114439 & -4.591944 \\
\hline-1.273171 & -5.108396 \\
\hline-2.211439 & -4.180530 \\
\hline-3.232440 & -4.380874 \\
\hline-2.028660 & -3.510382 \\
\hline-3.215892 & -3.086864 \\
\hline-3.191249 & -2.001988 \\
\hline 1.844186 & -3.366536 \\
\hline 1.933878 & -2.369132 \\
\hline 1.236971 & 2.289810 \\
\hline 2.913499 & 1.137439 \\
\hline 0.470744 & 0.596742 \\
\hline 2.610742 & 1.959196 \\
\hline 4.614517 & 2.434593 \\
\hline 3.551192 & 2.683849 \\
\hline 3.872752 & 4.301608 \\
\hline 3.130933 & 3.737426 \\
\hline 1.451135 & 4.890388 \\
\hline 1.768723 & 4.070758 \\
\hline-0.244688 & 3.595353 \\
\hline 0.821133 & 3.349270 \\
\hline 0.218884 & 1.555280 \\
\hline-0.836511 & 1.885799 \\
\hline-2.476429 & 0.47098 \\
\hline
\end{tabular}

A

$\begin{array}{rr}\text { Mn } & 0.498290 \\ \mathrm{~N} & -1.374248 \\ \mathrm{~N} & 0.882720 \\ \mathrm{C} & -2.981065 \\ \mathrm{C} & -1.357156 \\ \mathrm{C} & -2.589147 \\ \mathrm{H} & -2.863171 \\ \mathrm{C} & -3.366626 \\ \mathrm{H} & -4.382942 \\ \mathrm{C} & -2.590635 \\ \mathrm{C} & -0.177871 \\ \mathrm{H} & -0.159180 \\ \mathrm{C} & -3.200243 \\ \mathrm{C} & -3.571140 \\ \mathrm{H} & -3.744458 \\ \mathrm{C} & -3.735971 \\ \mathrm{C} & -3.527187 \\ \mathrm{H} & -3.654451 \\ \mathrm{C} & -3.158113 \\ \mathrm{C} & -2.956222 \\ \mathrm{H} & -2.024538 \\ \mathrm{H} & -2.917573 \\ \mathrm{H} & -3.778192 \\ \mathrm{H} & 2.102484 \\ \mathrm{H} & 0.633920 \\ \mathrm{H} & 1.637904\end{array}$

$-1.097385$

$-0.251171$

$-0.472680$

$-0.370590$

0.290577

0.939515

1. 444554

0.798900

1.161036

0.059040

0.150957

0.559265

$-1.750328$

$-2.134347$

$-3.198263$

$-1.196354$

0.161053

0.910088

0.593739

2. 074187

2. 273714

2. 633956

2. 502255

$-4.006902$

$-3.006666$

2. 368518
$-0.614552$

$-1.100209$

$-2.677034$

0.813357

$-2.388152$

$-2.658054$

$-3.586142$

$-1.496978$

$-1.334431$

$-0.557696$

$-3.169321$

$-4.192205$

2. 395133

2. 600831

3. 432834

3. 132319

3. 924259

1. 842947

1.586257

1. 029471

2. 535648

0.984870

$-3.948103$

$-4.123049$

$-3.814700$
1. 093982
3.319854

$-3.553386$

$-3.065992$

$-2.006326$

$-3.519107$

$-5.073184$

$-4.263180$

$-4.128434$

$-3.361197$

2. 076816

2. 873105

4.070537

4. 693258

4. 475841

5.411443

3. 681143

3. 991166

2. 478062

1. 610342

1. 391118

2. 472481

2. 143073

2. 388057

3. 704981

1. 507319

3. 545330

5. 359257

4. 462171

4. 957237

4.234040

2. 918732

3. 087376

1. 269559

2. 165175

1. 411954

0.547046

2. 673105

1. 170433

2. 507950

0.088588

3. 604134

1. 516475

4. 902466

1.504174

5.093422

3.443761

3. 992949

5.759214

6.102785

4. 167675

$\mathbf{T S}_{\mathrm{AB}}$

$\begin{array}{rr}\text { Mn } & 0.360171 \\ \mathrm{~N} & -1.611542 \\ \mathrm{~N} & 0.726439 \\ \mathrm{C} & -3.249184 \\ \mathrm{C} & -1.653171 \\ \mathrm{C} & -3.000513 \\ \mathrm{H} & -3.338222 \\ \mathrm{C} & -3.793317 \\ \mathrm{H} & -4.882574 \\ \mathrm{C} & -2.907889 \\ \mathrm{C} & -0.423626 \\ \mathrm{H} & -0.435239 \\ \mathrm{C} & -3.971870 \\ \mathrm{C} & -4.276353 \\ \mathrm{H} & -4.827264 \\ \mathrm{C} & -3.894670 \\ \mathrm{C} & -3.196058 \\ \mathrm{H} & -2.910466 \\ \mathrm{C} & -2.865943 \\ \mathrm{C} & -2.149103 \\ \mathrm{H} & -1.081304 \\ \mathrm{H} & -2.195118 \\ \mathrm{H} & -2.594762 \\ \mathrm{H} & 1.984791\end{array}$
2.639844

$-2.486579$

$-2.803481$

$-3.005396$

$-3.755295$

$-2.216209$

$-0.784477$

$-1.643268$

$-2.304524$

$-0.582962$

0.563692

0.388925

1. 267049

$-0.877613$

$-0.991907$

$-1.998926$

$-2.992391$

$-1.874993$

$-3.080832$

$-3.185891$

1. 939440

1. 916224

1. 675132

1. 910717

0.514058

2. 162204

3. 323121

2. 946751

3. 867779

3. 253055

3.014650

2. 774248

1. 600255

1.985562

0.840824

0.485360

$-4.143962$

$-3.057967$

$-5.484510$

$-4.077027$

$-6.270994$

$-2.522784$

$-5.735898$

$-5.926889$

$-4.408590$

$-7.306328$

$-3.626552$

$-6.347774$

$-3.981992$

$-2.592839$

$-0.635395$

$-0.379468$

$-0.136796$

$-0.720771$

$-0.095065$

0.016369

0.241427

$-0.217033$

$-0.202089$

$-0.453181$

0.018981

0.247609

$-1.893372$

$-2.126717$

$-3.037502$

$-1.233576$

$-0.069820$

0.656122

0.205645

1. 498838

1. 337156

2. 200862

1.992767

$-3.162396$
$-3.312494$

$-0.924312$

0.013849

$-0.227550$

0.336438

4. 813370

5.502782

4.824692

5.267223

$-3.450101$

$-3.720886$

$-4.443158$

$-4.650856$

$-4.886433$

$-5.444043$

$-4.606014$

$-4.949944$

$-3.886768$

$-3.610129$

$-2.530732$

$-3.231193$

$-2.178087$

2. 365829

0.655104

0.465124

1. 707799

1. 909298

2. 410869

4. 314106

3.767982

5.480873

4. 426683

4.229614

3. 729292

1. 671867

2.273556

0.406747

0.890112

$-0.020960$

1. 191831

$-0.414813$

2. 273593

$-0.387368$

$-0.037969$

0.034481

$-0.737385$

0.420430

$-0.690385$

0.062945

0.746119

$-0.744827$

$-1.402318$

$-2.831691$

0.449865

$-2.769888$

$-3.199687$

$-4.212925$

$-2.064340$

$-2.006401$

$-0.972243$

$-3.473209$

$-4.550478$

0.806521

2.161901

2. 428666

3. 178308

2. 803416

3. 575501

1. 463770

1.128589

0.890678

1. 977749

0.248053

$-5.117181$ 


\begin{tabular}{|c|c|}
\hline $\mathrm{H}$ & 0.561691 \\
\hline $\mathrm{H}$ & 1.498062 \\
\hline $\mathrm{H}$ & 3.208910 \\
\hline $\mathrm{H}$ & -5.303841 \\
\hline $\mathrm{C}$ & -4.420289 \\
\hline $\mathrm{H}$ & -3.633569 \\
\hline $\mathrm{H}$ & -4.697883 \\
\hline $\mathrm{H}$ & -4.987007 \\
\hline $\mathrm{H}$ & -4.562702 \\
\hline $\mathrm{C}$ & -4.210736 \\
\hline $\mathrm{H}$ & -3.315339 \\
\hline C & 1.964670 \\
\hline C & 2.833865 \\
\hline $\mathrm{C}$ & 4.080122 \\
\hline $\mathrm{H}$ & 4.750982 \\
\hline $\mathrm{C}$ & 4.467497 \\
\hline $\mathrm{H}$ & 5.442352 \\
\hline $\mathrm{C}$ & 3.603121 \\
\hline $\mathrm{H}$ & 3.904715 \\
\hline C & 2.343671 \\
\hline $\mathrm{C}$ & 1.438157 \\
\hline $\mathrm{H}$ & 1.042086 \\
\hline $\mathrm{C}$ & 2.415796 \\
\hline $\mathrm{H}$ & 2.197901 \\
\hline C & 2.651367 \\
\hline $\mathrm{H}$ & 3.770823 \\
\hline 0 & 1.490504 \\
\hline $\mathrm{C}$ & 3.741799 \\
\hline $\mathrm{H}$ & 5.600193 \\
\hline $\mathrm{C}$ & 4.759640 \\
\hline $\mathrm{H}$ & 5.497400 \\
\hline $\mathrm{C}$ & 4.703144 \\
\hline $\mathrm{H}$ & 3.562958 \\
\hline C & 3.618234 \\
\hline $\mathrm{H}$ & 1.749199 \\
\hline C & 2.598872 \\
\hline C & 1.557795 \\
\hline $\mathrm{H}$ & 0.590035 \\
\hline $\mathrm{C}$ & 2.825401 \\
\hline $\mathrm{Si}$ & 1.102117 \\
\hline $\mathrm{C}$ & 3.192338 \\
\hline $\mathrm{H}$ & 0.440162 \\
\hline C & 4.479469 \\
\hline $\mathrm{H}$ & 1.767963 \\
\hline $\mathrm{C}$ & 5.429520 \\
\hline $\mathrm{H}$ & 2.458366 \\
\hline $\mathrm{C}$ & 5.092464 \\
\hline $\mathrm{H}$ & 4.747754 \\
\hline $\mathrm{C}$ & 3.803324 \\
\hline $\mathrm{H}$ & 6.436799 \\
\hline $\mathrm{H}$ & 5.835121 \\
\hline $\mathrm{H}$ & 3.545784 \\
\hline
\end{tabular}

\begin{tabular}{|c|c|}
\hline-2.104562 & -5.240978 \\
\hline 2.643246 & -2.756071 \\
\hline 2.908160 & -2.326818 \\
\hline-2.545195 & -0.794444 \\
\hline-2.900439 & -0.233678 \\
\hline-3.102490 & -0.979823 \\
\hline-3.855370 & 0.242919 \\
\hline-2.290876 & 4.734935 \\
\hline-0.607361 & 5.152607 \\
\hline-1.514763 & 4.631437 \\
\hline-1.869139 & 5.175839 \\
\hline-0.085023 & -3.538361 \\
\hline 1.015899 & -3.307079 \\
\hline 1.034935 & -3.961404 \\
\hline 1.885636 & -3.794076 \\
\hline-0.008100 & -4.815822 \\
\hline 0.021911 & -5.314084 \\
\hline-1.091806 & -5.025367 \\
\hline-1.914210 & -5.684696 \\
\hline-1.154471 & -4.396115 \\
\hline-2.348245 & -4.613891 \\
\hline-2.737834 & -3.658902 \\
\hline 2.145495 & -2.393674 \\
\hline 1.783496 & -1.371576 \\
\hline 0.723717 & 2.589935 \\
\hline 1.197854 & 0.807451 \\
\hline 0.296847 & 0.482844 \\
\hline 1.284256 & 1.897739 \\
\hline 2.390925 & 2.047057 \\
\hline 1.952986 & 2.596902 \\
\hline 2.583954 & 4.541307 \\
\hline 2.062274 & 3.996494 \\
\hline 1.590337 & 5.785429 \\
\hline 1.502630 & 4.694746 \\
\hline 0.411710 & 4.540851 \\
\hline 0.840289 & 3.994689 \\
\hline-0.024504 & 1.844805 \\
\hline 0.127728 & 2.373509 \\
\hline-3.554642 & 0.636430 \\
\hline-2.831484 & 0.494029 \\
\hline-4.444259 & 1.684808 \\
\hline-3.450761 & 1.721170 \\
\hline-4.993419 & 1.744955 \\
\hline-1.139653 & 1.988417 \\
\hline-4.661658 & 0.761450 \\
\hline-4.701194 & 2.456886 \\
\hline-3.783729 & -0.284541 \\
\hline-5.676572 & 2.557759 \\
\hline-3.238835 & -0.347873 \\
\hline-5.089140 & 0.810929 \\
\hline-3.527977 & -1.047435 \\
\hline-2.557962 & -1.167642 \\
\hline
\end{tabular}

$-1.061387$ $-0.278361$ $-0.229591$ $-0.753152$ 0.408699 0.994258 1.582825

0.658971

0.921799

$-0.128750$

0.414999

0.942961

$-2.157218$

$-2.723377$

$-3.806774$

$-1.949265$

$-0.564843$

0.063164

0.047788

1. 546912

2.019410

2. 017545

2. 261847

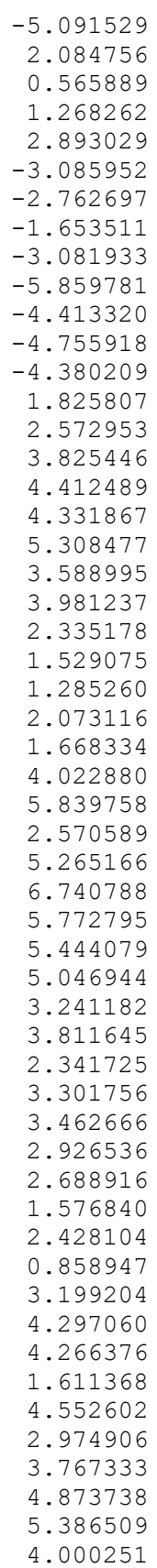

C

$-0.802873$

$-2.708562$

$-0.212497$

$-4.578904$

$-2.586608$

$-3.871148$

$-4.082592$

$-4.795279$

$-5.883582$

$-4.048557$

$-1.285551$

$-1.180128$

$-5.366243$

$-5.865143$

$-6.462599$

$-5.622899$

$-4.859319$

$-4.674893$

$-4.333095$

$-3.548428$

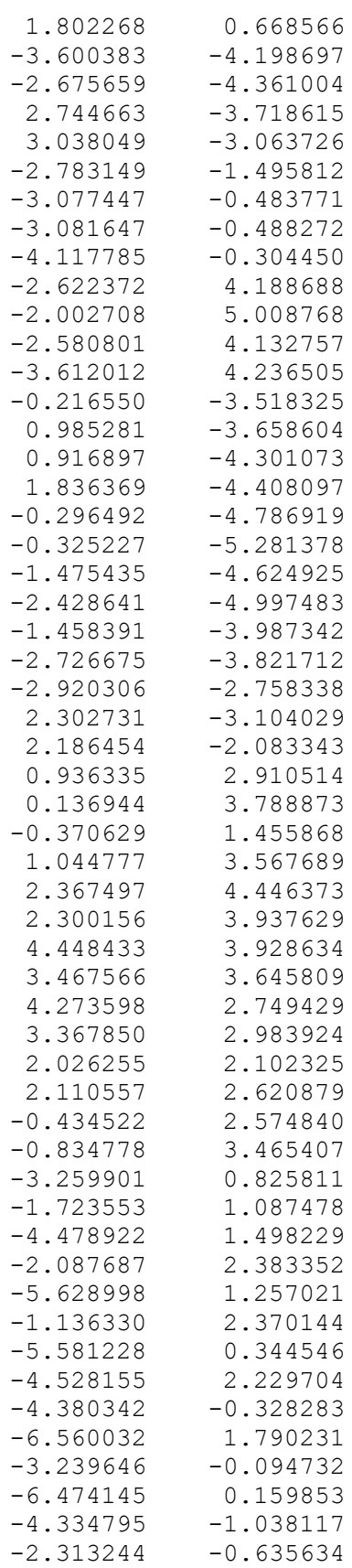

$-0.028950$

0.481058

1. 013305

$-0.307808$

1.140946

1. 427789

1. 945347

0.916520

0.962983

0.342239

1. 398008

1. 931789

$-1.489726$

$-2.089332$

$-3.005672$

$-1.550604$

$-0.371077$

0.081842

0.261420

1. 546021
$-0.601856$

$-1.363439$

$-2.426566$

0.105103

$-2.589474$

$-3.117784$

$-2.193424$

$-2.253585$

$-1.122951$

$-3.101165$

$-4.059070$

0.000791

1. 173516

1. 084297

2. 449465

2. 530784

3. 513354

1. 388991

1. 560455
$-4.055292$ 


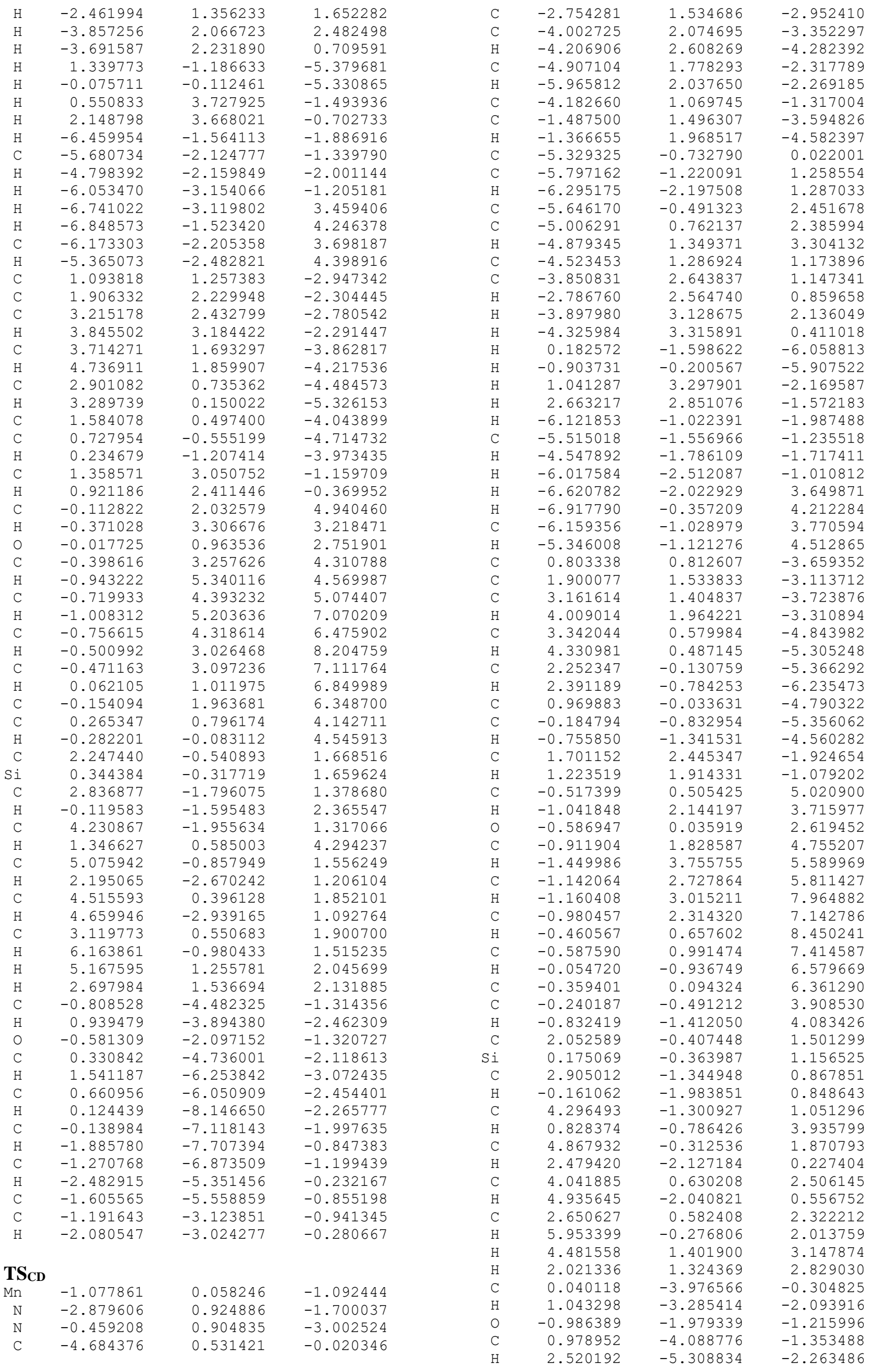




$\begin{array}{lr}\mathrm{C} & 1.796853 \\ \mathrm{H} & 2.325513 \\ \mathrm{C} & 1.686478 \\ \mathrm{H} & 0.659069 \\ \mathrm{C} & 0.751187 \\ \mathrm{H} & -0.798420 \\ \mathrm{C} & -0.066951 \\ \mathrm{C} & -0.849016 \\ \mathrm{H} & -1.728469\end{array}$

-5.223875
-7.141102
-6.254969
-6.953097
-6.149119
-4.928541
-5.015348
-2.781540
-2.934928

$$
\begin{array}{r}
-1.445402 \\
-0.570486 \\
-0.494873 \\
1.288074 \\
0.550204 \\
1.458094 \\
0.645568 \\
-0.194768 \\
0.468439
\end{array}
$$

D

$\begin{array}{rr}\text { Mn } & -1.168662 \\ \mathrm{~N} & -2.924159 \\ \mathrm{~N} & -0.489471 \\ \mathrm{C} & -4.752220 \\ \mathrm{C} & -2.769161 \\ \mathrm{C} & -3.995249 \\ \mathrm{H} & -4.174146 \\ \mathrm{C} & -4.916140 \\ \mathrm{H} & -5.965595 \\ \mathrm{C} & -4.223436\end{array}$

$-1.497153$

$-1.356423$

$-5.369043$

$-5.863278$

$-6.336774$

$-5.769344$

$-5.153639$

$-5.066330$

$-4.645155$

$-3.993976$

$-2.930363$

$-4.045660$

$-4.482039$

0.116506

$-0.958961$

1.047232

2. 659461

$-6.037779$

$-5.493603$

$-4.503240$

$-6.030796$

$-6.371039$

$-7.380785$

$-6.343190$

$-5.757196$

0.774011

1.880243

3. 141714

3. 996351

3. 312930

4. 301694

2. 213687

2. 344899

0.931061

$-0.236111$

$-0.798546$

1. 692072

1.201974

$-0.536209$

$-1.253361$

$-0.763750$

$-0.974118$

$-1.397895$

$-1.056492$

$-0.770364$

$-0.705594$

0.008891

$-0.269211$

0.160467

$-0.183680$

$-0.439382$

$-1.158887$

1. 936626

0.079697

2. 806152
$-0.036803$

0.911949

0.824866

0.602826

1. 509403

2. 089125

2. 622208

1. 833589

2. 129703

1. 106588

1. 432802

1. 889660

$-0.675157$

$-1.126799$

$-2.115523$

$-0.347799$

0.914742

1. 537449

1. 405355

2. 771970

2.703591

3. 276341

3. 422514

$-1.764059$

$-0.357138$

3. 222246

2. 769472

$-1.033347$

$-1.549147$

$-1.819006$

$-2.483593$

$-1.939589$

$-0.480361$

$-0.837830$

$-0.473858$

0.692794

1. 414080

1. 245755

1. 805686

0.381895

0.258925

$-0.328398$

$-1.011498$

$-0.191912$

$-0.989047$

$-1.485810$

2. 367577

1. 873899

0.459566

2.001347

$-0.146279$

1.760596

3.747481

2. 737584

3. 185462

2. 423819

0.868798

1.123623

$-0.861688$

0.149740

$-0.629730$

$-1.438294$

$-0.434553$

$-0.344986$

$-1.207759$
$-1.071160$

$-1.695418$

$-2.970324$

$-0.022138$

$-2.950092$

$-3.363143$

$-4.298747$

$-2.332186$

$-2.292692$

$-1.322181$

$-3.579257$

$-4.571666$

0.049403

1. 288171

1. 339348

2. 456483

2. 362892

3. 262095

1.145750

1.089713

0.797543

2. 068485

0.342556

$-5.962507$

$-5.809159$

$-2.209298$

$-1.591465$

$-1.992027$

$-1.181424$

$-1.589358$

$-0.950145$

3. 813240

3.910106

3. 768801

4.629820

$-3.617383$

$-3.091653$

$-3.692686$

$-3.295467$

$-4.784470$

$-5.239075$

$-5.287498$

$-6.134835$

$-4.720084$

$-5.262488$

$-4.453008$

$-1.933922$

$-1.073514$

5.048206

3.712775

2. 678273

4. 742596

5.498343

5.750642

7.856778

7.072538

8. 412587

7.384165

6.627702

6. 378961

3. 993326

4. 228799

1. 645517

1. 234214

0.835673
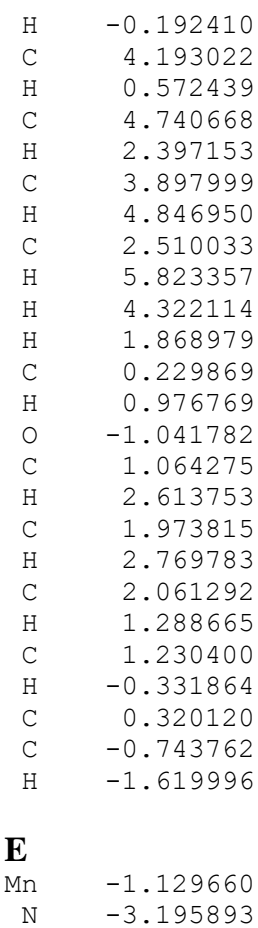

$\mathrm{N}$
$\mathrm{N}$

$-1.129660$

$-3.195893$

$-1.294058$

$-4.353757$

$-3.565027$

$-4.959108$

$-5.526022$

$-5.443849$

$-6.475933$

$-4.335251$

$-2.563056$

$-2.849174$

$-3.999987$

$-4.040649$

$-3.779036$

$-4.421317$

$-4.782206$

$-5.091481$

$-4.760416$

$-5.162955$

$-4.460819$

$-5.201261$

$-6.162596$

$-1.194637$

$-2.424617$

$-0.054291$

1. 729925

$-4.240077$

$-3.609626$

$-2.561002$

$-3.705408$

$-3.404619$

$-4.824969$

$-4.427234$

$-5.036160$

$-0.311651$

0.771841

1. 767211

2.597656

1. 707131

2. 492512

0.642793

0.600870

$-0.380524$

$-1.494294$

$-1.751706$

0.835544

0.873013

$-0.314369$

$-2.130542$

$-1.198440$

$-1.078706$

$-0.404109$

$-1.833624$

0.380479

$-1.810827$

0.364136

$-0.393269$

1. 004346

0.986025

$-3.939910$

$-3.092429$ 


$\begin{array}{rr}\mathrm{H} & -0.818413 \\ \mathrm{O} & -0.965185 \\ \mathrm{C} & -0.326092 \\ \mathrm{H} & 0.281087 \\ \mathrm{C} & 0.293244 \\ \mathrm{H} & 1.405739 \\ \mathrm{C} & 0.923631 \\ \mathrm{H} & 1.429724 \\ \mathrm{C} & 0.935946 \\ \mathrm{H} & 0.341056 \\ \mathrm{C} & 0.324622 \\ \mathrm{C} & -1.021812 \\ \mathrm{H} & -2.098016 \\ \mathrm{C} & 1.118544 \\ \mathrm{Si} & -0.152140 \\ \mathrm{C} & 1.414164 \\ \mathrm{H} & -0.384955 \\ \mathrm{C} & 2.367185 \\ \mathrm{H} & -0.608564 \\ \mathrm{C} & 3.060607 \\ \mathrm{H} & 0.896660 \\ \mathrm{C} & 2.805260 \\ \mathrm{H} & 2.578320 \\ \mathrm{C} & 1.840922 \\ \mathrm{H} & 3.811919 \\ \mathrm{H} & 3.361071 \\ \mathrm{H} & 1.667549 \\ \mathrm{C} & 1.198063 \\ \mathrm{H} & 2.126670 \\ \mathrm{O} & -0.230057 \\ \mathrm{C} & 2.277942 \\ \mathrm{H} & 4.354375 \\ \mathrm{C} & 3.523087 \\ \mathrm{H} & 4.673137 \\ \mathrm{C} & 3.703819 \\ \mathrm{H} & 2.758333 \\ \mathrm{C} & 2.628564 \\ \mathrm{H} & 0.553957 \\ \mathrm{C} & 1.388208 \\ \mathrm{H} & -0.161181 \\ \mathrm{H} & \\ \mathrm{H} & \\ \mathrm{H} & \\ \mathrm{H} & \\ \mathrm{H} & -93579\end{array}$

\section{TSEF}

$\begin{array}{cl}\text { Mn } & -1.127092 \\ \mathrm{~N} & -3.194868 \\ \mathrm{~N} & -1.296155 \\ \mathrm{C} & -4.350141 \\ \mathrm{C} & -3.567555 \\ \mathrm{C} & -4.963432 \\ \mathrm{H} & -5.533193 \\ \mathrm{C} & -5.445801 \\ \mathrm{H} & -6.478434 \\ \mathrm{C} & -4.334071 \\ \mathrm{C} & -2.566950 \\ \mathrm{H} & -2.856531 \\ \mathrm{C} & -3.977842 \\ \mathrm{C} & -4.016873 \\ \mathrm{H} & -3.740594 \\ \mathrm{C} & -4.414270 \\ \mathrm{C} & -4.792775 \\ \mathrm{H} & -5.114532 \\ \mathrm{C} & -4.772586 \\ \mathrm{C} & -5.192682 \\ \mathrm{H} & -4.496937 \\ \mathrm{H} & -5.237010 \\ \mathrm{H} & -6.193381 \\ \mathrm{H} & -1.192263 \\ \mathrm{H} & -2.423700 \\ \mathrm{H} & -0.059023 \\ \mathrm{H} & 1.725038 \\ \mathrm{H} & -4.208083 \\ \mathrm{C} & -3.570948 \\ \mathrm{H} & -2.528101 \\ \mathrm{H} & -3.640910 \\ \mathrm{H} & -3.396529 \\ \mathrm{H} & -4.812381 \\ & \end{array}$

\begin{tabular}{rr}
3.925584 & 2.536997 \\
1.341708 & 2.491099 \\
3.971720 & 3.513709 \\
6.038199 & 3.286046 \\
5.157620 & 3.937334 \\
6.145799 & 5.520959 \\
5.219198 & 5.192085 \\
4.121900 & 6.996972 \\
4.085003 & 6.020034 \\
2.009344 & 6.239419 \\
2.894661 & 5.592609 \\
1.553153 & 3.920926 \\
1.618634 & 4.167139 \\
-0.719507 & 2.542269 \\
0.240028 & 1.535538 \\
-2.020203 & 2.049262 \\
-4.163190 & -1.263513 \\
-2.827993 & 2.689283 \\
0.683475 & 4.461973 \\
-2.341722 & 3.810016 \\
-2.383440 & 1.148819 \\
-1.044259 & 4.291480 \\
-3.832123 & 2.306130 \\
-0.240649 & 3.667808 \\
-2.968092 & 4.303243 \\
-0.660851 & 5.153993 \\
0.772042 & 4.047825 \\
-3.320698 & -2.443766 \\
-1.718228 & -1.343203 \\
-2.153235 & -0.809065 \\
-2.484846 & -2.109739 \\
-1.973005 & -2.475748 \\
-2.633890 & -2.746734 \\
-3.740207 & -4.222143 \\
-3.623546 & -3.725393 \\
-5.240685 & -4.828430 \\
-4.465189 & -4.065009 \\
-4.974692 & -3.697673 \\
-4.313898 & -3.428299 \\
-3.187497 & -1.755763 \\
-3.079622 & -2.551192 \\
& \\
\hline
\end{tabular}

$-0.421387$

$-0.071210$

0.635929

$-0.948917$

0.617764

0.871948

1.382108

0.331471

0.322274

$-0.244021$

0.944309

1.466228

$-2.322263$

$-2.965282$

$-4.026126$

$-2.297580$

$-0.947633$

$-0.406940$

$-0.261358$

1.194970

1.809916

1.611803

1.325751

$-1.400540$

$-0.231002$

3.065167

3.080775

$-2.884008$

$-3.118032$

$-2.901596$

$-4.200369$

$-3.092128$

$-4.035785$

5.036143

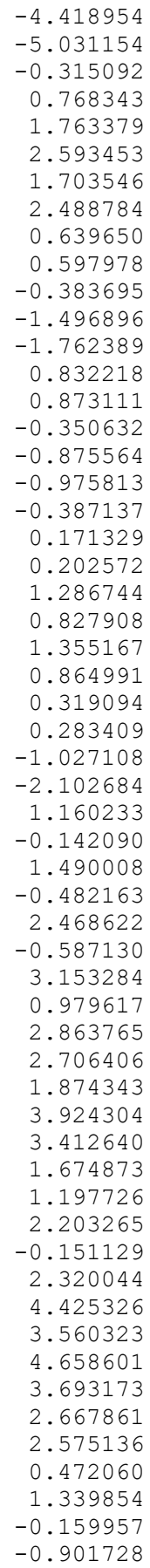

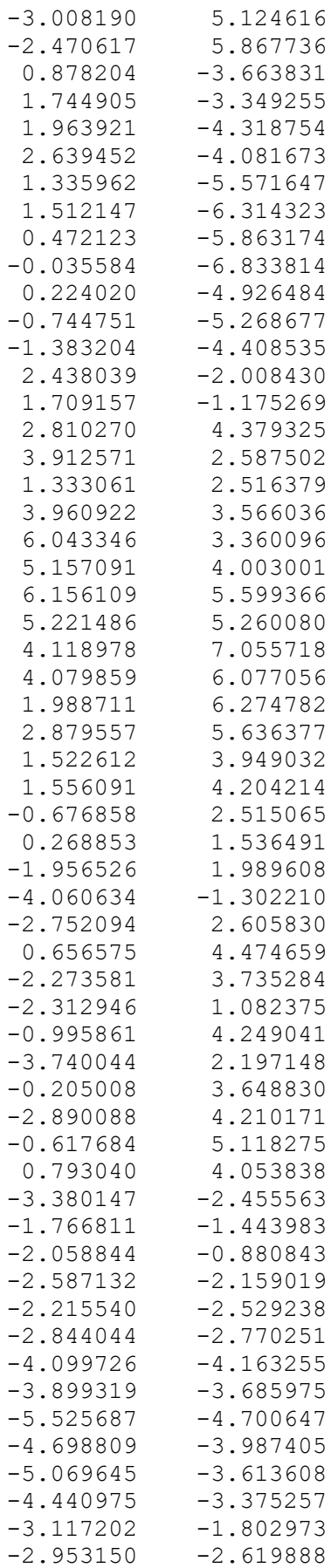

5.124616 5.867736

$-3.663831$

$-3.349255$

$-4.318754$

$-4.081673$

$-5.571647$

$-6.314323$

$-5.863174$

$-6.833814$

$-4.926484$

$-5.268677$

$-4.408535$

$-2.008430$

$-1.175269$

4. 379325

2. 587502

2. 516379

3. 566036

3.360096

4. 003001

5.599366

5.260080

7.055718

6.077056

6.274782

5.636377

3. 949032

4. 204214

2. 515065

1.536491

1. 989608

$-1.302210$

2. 605830

4.474659

3.735284

1. 082375

4. 249041

2. 197148

3. 648830

4. 210171

5. 118275

4.053838

$-2.455563$

$-1.443983$

$-0.880843$

$-2.159019$

$-2.529238$

$-2.770251$

$-4.163255$

$-3.685975$

$-4.700647$

$-3.987405$

$-3.613608$

$-3.375257$

$-1.802973$

$-2.619888$

0.463979

0.509047

1.275204

$-0.320983$

1.031626

1.108886

1.467805

0.629236

0.524335

0.264827

1.406872

1.827402

$-1.593534$

$-2.132603$

$-3.121061$

$-1.452401$

$-0.198605$
$-0.965088$

$-1.334785$

$-2.983732$

0.624060

$-2.602795$

$-2.802432$

$-3.700946$

$-1.622798$

$-1.415562$

$-0.736631$

$-3.427973$

$-4.426706$

0.905975

2. 200279

2. 403600

3. 230806

2. 931224 


\begin{tabular}{|c|c|c|c|}
\hline $\mathrm{H}$ & -6.095073 & 0.351875 & 3.719462 \\
\hline C & -5.472148 & 0.378177 & 1.652236 \\
\hline C & -6.107629 & 1.733406 & 1.411716 \\
\hline $\mathrm{H}$ & -5.465394 & 2.390514 & 0.801751 \\
\hline $\mathrm{H}$ & -6.309893 & 2.241408 & 2.369313 \\
\hline $\mathrm{H}$ & -7.071632 & 1.648157 & 0.878047 \\
\hline $\mathrm{H}$ & -0.312038 & -0.863912 & -6.200748 \\
\hline $\mathrm{H}$ & -1.792521 & 0.072871 & -5.912285 \\
\hline $\mathrm{H}$ & -0.711209 & 3.988244 & -2.180573 \\
\hline $\mathrm{H}$ & 1.034431 & 4.155489 & -1.843844 \\
\hline $\mathrm{H}$ & -3.898523 & -2.264766 & -1.152193 \\
\hline $\mathrm{C}$ & -3.468244 & -2.403414 & -0.146963 \\
\hline $\mathrm{H}$ & -2.396564 & -2.126558 & -0.203133 \\
\hline $\mathrm{H}$ & -3.502147 & -3.477979 & 0.100086 \\
\hline $\mathrm{H}$ & -4.313913 & -1.617020 & 5.284765 \\
\hline $\mathrm{H}$ & -4.964047 & -3.130535 & 4.614104 \\
\hline $\mathrm{C}$ & -5.095744 & -2.035693 & 4.623632 \\
\hline $\mathrm{H}$ & -6.068665 & -1.810288 & 5.093086 \\
\hline C & -0.280475 & 1.620453 & -3.799580 \\
\hline $\mathrm{C}$ & 0.569666 & 2.667951 & -3.349249 \\
\hline C & 1.703777 & 2.993219 & -4.114926 \\
\hline $\mathrm{H}$ & 2.357234 & 3.804851 & -3.775166 \\
\hline $\mathrm{C}$ & 2.002203 & 2.296100 & -5.295023 \\
\hline $\mathrm{H}$ & 2.891664 & 2.556929 & -5.878037 \\
\hline $\mathrm{C}$ & 1.164220 & 1.255087 & -5.717806 \\
\hline $\mathrm{H}$ & 1.404070 & 0.696381 & -6.629907 \\
\hline C & 0.015089 & 0.894124 & -4.986133 \\
\hline C & -0.847216 & -0.261857 & -5.448416 \\
\hline $\mathrm{H}$ & -1.122551 & -0.926536 & -4.610837 \\
\hline $\mathrm{C}$ & 0.239504 & 3.432288 & -2.087517 \\
\hline $\mathrm{H}$ & 0.125823 & 2.755171 & -1.217347 \\
\hline C & 0.016962 & 0.461730 & 4.666270 \\
\hline $\mathrm{H}$ & -1.111769 & 1.882624 & 3.500023 \\
\hline O & -0.225017 & 0.016979 & 2.278508 \\
\hline $\mathrm{C}$ & -0.764794 & 1.619013 & 4.503314 \\
\hline $\mathrm{H}$ & -1.710051 & 3.313566 & 5.471016 \\
\hline C & -1.098424 & 2.415512 & 5.612979 \\
\hline $\mathrm{H}$ & -0.915491 & 2.684329 & 7.761519 \\
\hline C & -0.654419 & 2.064228 & 6.897215 \\
\hline $\mathrm{H}$ & 0.477996 & 0.622808 & 8.065486 \\
\hline C & 0.127381 & 0.907381 & 7.067236 \\
\hline $\mathrm{H}$ & 1.067261 & -0.789473 & 6.099469 \\
\hline $\mathrm{C}$ & 0.458995 & 0.113049 & 5.960021 \\
\hline C & 0.402619 & -0.417530 & 3.486841 \\
\hline $\mathrm{H}$ & 0.106126 & -1.463936 & 3.708639 \\
\hline $\mathrm{C}$ & 2.145338 & 0.161559 & 0.629944 \\
\hline Si & 0.313123 & -0.390811 & 0.705780 \\
\hline $\mathrm{C}$ & 3.069344 & -0.466199 & -0.242283 \\
\hline $\mathrm{H}$ & 2.117648 & -2.566907 & 1.457631 \\
\hline C & 4.389471 & -0.002151 & -0.367887 \\
\hline $\mathrm{H}$ & 1.508796 & -0.416570 & 3.382617 \\
\hline C & 4.817531 & 1.110302 & 0.376637 \\
\hline $\mathrm{H}$ & 2.753185 & -1.336801 & -0.830530 \\
\hline C & 3.918758 & 1.754477 & 1.243848 \\
\hline $\mathrm{H}$ & 5.085573 & -0.509484 & -1.045538 \\
\hline C & 2.600115 & 1.284881 & 1.364650 \\
\hline $\mathrm{H}$ & 5.846390 & 1.474223 & 0.280165 \\
\hline $\mathrm{H}$ & 4.246684 & 2.621627 & 1.828508 \\
\hline $\mathrm{H}$ & 1.911155 & 1.797441 & 2.047439 \\
\hline C & 1.415794 & -4.267114 & 0.332641 \\
\hline $\mathrm{H}$ & 0.615678 & -3.445878 & -1.494915 \\
\hline O & 0.323003 & -2.089881 & 0.478279 \\
\hline C & 1.092186 & -4.316859 & -1.035250 \\
\hline $\mathrm{H}$ & 1.109284 & -5.492686 & -2.857184 \\
\hline C & 1.369412 & -5.468197 & -1.792984 \\
\hline $\mathrm{H}$ & 2.188774 & -7.480691 & -1.781789 \\
\hline C & 1.974285 & -6.583049 & -1.191983 \\
\hline $\mathrm{H}$ & 2.768748 & -7.406837 & 0.655573 \\
\hline $\mathrm{C}$ & 2.299746 & -6.540784 & 0.175669 \\
\hline $\mathrm{H}$ & 2.272541 & -5.368150 & 1.998632 \\
\hline C & 2.020552 & -5.392436 & 0.931078 \\
\hline C & 1.147810 & -3.027414 & 1.172888 \\
\hline $\mathrm{H}$ & 0.652568 & -3.334459 & 2.117321 \\
\hline
\end{tabular}

G

$\begin{array}{lll}-0.215716 & 0.214514 & -1.502074\end{array}$

\begin{tabular}{|c|c|c|}
\hline-2.249415 & 0.093953 & -2.037637 \\
\hline-0.044305 & 0.383162 & -3.697894 \\
\hline-3.739733 & -0.129346 & -0.035703 \\
\hline-2.391093 & 0.027179 & -3.427360 \\
\hline-3.740556 & -0.249704 & -3.763698 \\
\hline-4.145846 & -0.351560 & -4.772021 \\
\hline-4.431430 & -0.362797 & -2.546422 \\
\hline-5.495583 & -0.557336 & -2.405622 \\
\hline-3.485820 & -0.147300 & -1.50279 \\
\hline-1.243556 & 0.209763 & -4.24186 \\
\hline-1.358382 & 0.191610 & -5.33669 \\
\hline-4.029956 & -1.337941 & 0.65506 \\
\hline-4.291485 & -1.288973 & 2.03756 \\
\hline-4.511808 & -2.225072 & 2.5658 \\
\hline-4.279535 & -0.080802 & 2.75837 \\
\hline-4.003744 & 1.105357 & 2.05267 \\
\hline-4.008484 & 2.061867 & 2.5902 \\
\hline-3.734150 & 1.105304 & 0.6715 \\
\hline-3.476124 & 2.418242 & -0.04045 \\
\hline-2.415932 & 2.527698 & -0.33272 \\
\hline-3.731177 & 3.272600 & 0.60777 \\
\hline-4.068250 & 2.499827 & -0.96857 \\
\hline 3.113332 & -1.999255 & -3.76284 \\
\hline 1.329672 & -2.066255 & -3.84031 \\
\hline-0.875127 & 2.511205 & -6.09354 \\
\hline 0.259145 & 3.820669 & -5.71541 \\
\hline-4.864797 & -2.718619 & -0.81530 \\
\hline-4.055936 & -2.670838 & -0.06524 \\
\hline-3.111907 & -2.861324 & -0.60529 \\
\hline-4.210280 & -3.497319 & 0.64748 \\
\hline-5.123207 & -0.933103 & 4.57310 \\
\hline-5.076874 & 0.853127 & 4.55455 \\
\hline-4.534800 & -0.058252 & 4.24948 \\
\hline-3.584860 & -0.073530 & 4.8151 \\
\hline 1.071533 & 0.687359 & -4.53329 \\
\hline 1.078406 & 1.854465 & -5.35015 \\
\hline 2.227264 & 2.122944 & -6.12221 \\
\hline 2.241911 & 3.025304 & -6.74456 \\
\hline 3.339295 & 1.271939 & -6.09898 \\
\hline 4.220592 & 1.500302 & -6.70750 \\
\hline 3.320707 & 0.130926 & -5.28311 \\
\hline 4.185959 & -0.541496 & -5.25848 \\
\hline 2.201561 & -0.176227 & -4.48873 \\
\hline 2.191659 & -1.412068 & -3.61956 \\
\hline 2.129422 & -1.148665 & -2.54575 \\
\hline-0.084824 & 2.825714 & -5.38782 \\
\hline-0.565130 & 2.937592 & -4.40122 \\
\hline 5.148289 & -0.062574 & 0.74358 \\
\hline 4.551359 & 0.635867 & -1.21036 \\
\hline 2.816176 & -0.403857 & 0.11411 \\
\hline 5.360346 & 0.608822 & -0.47476 \\
\hline 6.740058 & 1.758249 & -1.69006 \\
\hline 6.590461 & 1.236923 & -0.73788 \\
\hline 8.582294 & 1.687093 & 0.00548 \\
\hline 7.624437 & 1.197126 & 0.21091 \\
\hline 8.218682 & 0.491909 & 2.17901 \\
\hline 7.420101 & 0.525850 & 1.42965 \\
\hline 6.034792 & -0.613346 & 2.64836 \\
\hline 6.190907 & -0.096635 & 1.69329 \\
\hline 3.840472 & -0.772528 & 1.04853 \\
\hline 3.525704 & -0.534003 & 2.08426 \\
\hline 1.069422 & -2.708587 & 0.53037 \\
\hline 1.172621 & -0.807218 & 0.38617 \\
\hline 0.076278 & -3.339417 & 1.31923 \\
\hline 1.473694 & 1.597501 & 2.14803 \\
\hline-0.040012 & -4.738814 & 1.36403 \\
\hline 4.003587 & -1.870826 & 1.01707 \\
\hline 0.835362 & -5.543605 & 0.61488 \\
\hline-0.611420 & -2.725983 & 1.91259 \\
\hline 1.826143 & -4.940873 & -0.17851 \\
\hline-0.812813 & -5.203366 & 1.98715 \\
\hline 1.935687 & -3.540681 & -0.22088 \\
\hline 0.745830 & -6.635020 & 0.64912 \\
\hline 2.515293 & -5.562233 & -0.76187 \\
\hline 2.716670 & -3.086101 & -0.84295 \\
\hline 0.253913 & 1.114257 & 3.85054 \\
\hline 0.342085 & -1.002276 & 4.26244 \\
\hline
\end{tabular}




\begin{tabular}{rr} 
O & 0.774372 \\
$\mathrm{C}$ & 0.171327 \\
$\mathrm{H}$ & -0.175647 \\
$\mathrm{C}$ & -0.117912 \\
$\mathrm{H}$ & -0.551626 \\
$\mathrm{C}$ & -0.327745 \\
$\mathrm{H}$ & -0.404930 \\
$\mathrm{C}$ & -0.245426 \\
$\mathrm{H}$ & 0.112555 \\
$\mathrm{C}$ & 0.043925 \\
$\mathrm{C}$ & 0.572361 \\
$\mathrm{H}$ & -0.258826 \\
$\mathrm{H}$ & 0.347872 \\
$\mathrm{C}$ & 1.746725 \\
$\mathrm{Si}$ & 1.321719 \\
$\mathrm{C}$ & 2.922752 \\
$\mathrm{H}$ & 2.549427 \\
$\mathrm{C}$ & 3.231647 \\
$\mathrm{H}$ & 0.670920 \\
$\mathrm{C}$ & 2.373010 \\
$\mathrm{H}$ & 3.608632 \\
$\mathrm{C}$ & 1.204546 \\
$\mathrm{H}$ & 4.145764 \\
$\mathrm{C}$ & 0.895390 \\
$\mathrm{H}$ & 2.616755 \\
$\mathrm{H}$ & 0.535697 \\
$\mathrm{H}$ & -0.015941 \\
\hline &
\end{tabular}

\section{$\mathbf{T S}_{\text {GH }}$}

\begin{tabular}{|c|c|}
\hline & \\
\hline $\mathrm{Mn}$ & -0.190812 \\
\hline $\mathrm{N}$ & -2.161568 \\
\hline $\mathrm{N}$ & 0.008588 \\
\hline $\mathrm{C}$ & -3.636636 \\
\hline $\mathrm{C}$ & -2.302617 \\
\hline C & -3.630125 \\
\hline $\mathrm{H}$ & -4.030453 \\
\hline $\mathrm{C}$ & -4.304313 \\
\hline $\mathrm{H}$ & -5.351510 \\
\hline $\mathrm{C}$ & -3.376421 \\
\hline C & -1.176186 \\
\hline $\mathrm{H}$ & -1.286790 \\
\hline $\mathrm{C}$ & -4.052899 \\
\hline C & -4.314592 \\
\hline $\mathrm{H}$ & -4.626043 \\
\hline C & -4.189124 \\
\hline C & -3.798107 \\
\hline $\mathrm{H}$ & -3.718995 \\
\hline $\mathrm{C}$ & -3.518999 \\
\hline $\mathrm{C}$ & -3.143914 \\
\hline $\mathrm{H}$ & -2.069591 \\
\hline $\mathrm{H}$ & -3.350438 \\
\hline $\mathrm{H}$ & -3.700636 \\
\hline $\mathrm{H}$ & 3.351617 \\
\hline $\mathrm{H}$ & 1.608680 \\
\hline $\mathrm{H}$ & -0.971425 \\
\hline $\mathrm{H}$ & 0.082878 \\
\hline $\mathrm{H}$ & -5.119237 \\
\hline $\mathrm{C}$ & -4.213634 \\
\hline $\mathrm{H}$ & -3.359485 \\
\hline $\mathrm{H}$ & -4.303319 \\
\hline $\mathrm{H}$ & -5.118038 \\
\hline $\mathrm{H}$ & -4.889633 \\
\hline $\mathrm{C}$ & -4.443073 \\
\hline $\mathrm{H}$ & -3.500151 \\
\hline $\mathrm{C}$ & 1.102578 \\
\hline $\mathrm{C}$ & 1.031881 \\
\hline $\mathrm{C}$ & 2.162314 \\
\hline $\mathrm{H}$ & 2.118653 \\
\hline $\mathrm{C}$ & 3.330503 \\
\hline $\mathrm{H}$ & 4.196260 \\
\hline $\mathrm{C}$ & 3.389264 \\
\hline $\mathrm{H}$ & 4.299749 \\
\hline $\mathrm{C}$ & 2.289004 \\
\hline $\mathrm{C}$ & 2.352156 \\
\hline $\mathrm{H}$ & 2.137700 \\
\hline $\mathrm{C}$ & -0.192160 \\
\hline
\end{tabular}

$\begin{array}{rr}-0.373561 & 1.996270 \\ -0.010680 & 4.690584 \\ -0.742978 & 6.701799 \\ 0.142202 & 6.058460 \\ 1.538065 & 7.666630 \\ 1.419813 & 6.600939 \\ 3.550495 & 6.178867 \\ 2.548402 & 5.765548 \\ 3.280122 & 3.756694 \\ 2.395709 & 4.401804 \\ 0.987747 & 2.369465 \\ 1.432024 & 1.778711 \\ 4.829002 & -2.070228 \\ 4.808117 & 0.327343 \\ 3.980706 & -1.310997 \\ 4.463708 & 1.037946 \\ 3.753777 & -2.135528 \\ 5.081863 & 2.260151 \\ 2.628880 & -1.111970 \\ 6.058849 & 2.792279 \\ 3.709388 & 0.634717 \\ 6.416206 & 2.099150 \\ 4.803284 & 2.795185 \\ 5.796158 & 0.877316 \\ 6.543594 & 3.743716 \\ 7.181831 & 2.506990 \\ 6.092279 & 0.344369\end{array}$

0.571825

0.163915

0.522441

$-0.043626$

0.005105

$-0.388699$

$-0.576307$

$-0.480550$

$-0.741467$

$-0.132869$

0.226564

0.137062

$-1.196306$

$-1.081418$

$-1.976570$

0.139288

1.271679

2.240906

1.205855

2.473485

2.495214

3. 362442

2. 572673

$-1.552540$

$-1.870989$

2.377216

3.788063

$-2.588025$

$-2.546471$

$-2.786622$

$-3.345628$

$-0.570716$

1.200116

0.229800

0.134215

0.837948

1.927690

2.201419

3.044233

1.434478

1.668859

0.373895

$-0.230355$

0.062882

$-1.088310$

$-0.751833$
$-2.048572$

$-3.733825$

$-0.029328$

$-3.432280$

$-4.738292$

$-2.514683$

$-2.356464$

$-1.488913$

$-4.263065$

$-5.353780$

0.695791

2.074404

2. 627288

2.761668

2.023183

2.531508

0.645691

$-0.094348$

$-0.360187$

0.523951

$-1.042193$

$-3.502453$

$-3.736354$

$-6.275176$

$-6.060581$

$-0.605322$

0.025869

$-0.629424$

0.780222

4.596604

4.527156

4.250293

4.820334

$-4.595436$

$-5.509557$

$-6.306555$

$-7.006088$

$-6.210203$

$-6.838422$

$-5.294077$

$-5.208555$

$-3.501452$

$-2.468296$

$-5.624240$
$-1.588155$

$-3.740603$

$-4.475752$

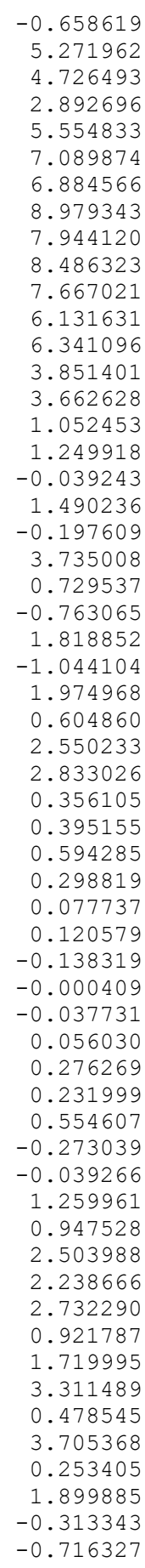

H

$-0.262029$

$-1.971820$

$-0.005355$

$-3.411284$

$-1.983641$

$-3.089005$

$-3.355750$

$-3.758819$

$-4.668604$

$-3.050900$

$-0.977334$

$-1.014976$

$-3.545861$

$-3.910997$

$-3.989356$

$-4.171036$
2.999724

$-0.145537$

0.880370

0.060939

0.559021

1.399559

0.847869

0.660385

0.434486

$-0.599153$

$-0.271861$

$-1.108004$

$-0.558587$

$-0.477194$

$-0.065739$

$-2.219956$

$-0.339626$

$-2.885406$

1.850873

$-4.274858$

$-1.577496$

$-5.028375$

$-2.311448$

$-4.386627$

$-4.771960$

$-2.996463$

$-6.112315$

$-4.970233$

$-2.510179$

1.204672

$-0.952989$

$-0.008992$

$-0.029017$

$-1.038998$

$-0.070788$

1.084163

1.118114

3.288942

2. 354573

3. 363225

2.396702

1.295762

1.884786

3.771801

4.193781

3.049021

4.190678

2.948491

5.032853

1. 254024

5.898607

3.531135

5.915716

5.018017

5.073797

6.560172

6.593549

5.114797

1.613069

0.377776

0.466889

0.863229

$-0.530108$

$-1.410050$

$-2.212517$

$-1.030203$

$-1.468965$

0.077716

$-0.428949$

$-1.099095$

0.216521

0.977160

0.472970

2.357182

$-4.642151$

0.965799

$-0.855327$

0.459342

$-0.217683$

$-1.496747$

$-0.572677$

$-0.027722$

0.249355

2. 083044

1. 433599

2. 714795

1. 788593

1. 387498

2. 399257

0.417698

0.536117

1.029343

2. 441271

0.905332

1. 465586

0.164873

1. 617148

$-0.448934$

1. 391703

$-0.327797$

0.068141

$-1.018931$

$-0.806138$

4.156676

4.252005

2. 058241

4. 829011

6.734589

6.223418

8. 045568

6.959548

6.855890

6.291644 


\begin{tabular}{|c|c|c|c|}
\hline C & -4.046643 & 2.975746 & 1.150521 \\
\hline $\mathrm{H}$ & -4.258569 & 4.048485 & 1.058986 \\
\hline $\mathrm{C}$ & -3.670268 & 2.261400 & -0.002648 \\
\hline C & -3.588020 & 2.997661 & -1.324765 \\
\hline $\mathrm{H}$ & -2.539409 & 3.200013 & -1.619613 \\
\hline $\mathrm{H}$ & -4.100828 & 3.971802 & -1.260891 \\
\hline $\mathrm{H}$ & -4.042570 & 2.418905 & -2.146682 \\
\hline $\mathrm{H}$ & 3.816033 & -0.483310 & -3.422087 \\
\hline $\mathrm{H}$ & 2.233884 & -1.297276 & -3.268695 \\
\hline $\mathrm{H}$ & -1.646220 & 0.886563 & -6.420893 \\
\hline $\mathrm{H}$ & -1.051576 & 2.372361 & -7.195184 \\
\hline $\mathrm{H}$ & -4.217745 & -1.856827 & 1.328873 \\
\hline C & -3.304384 & -1.269509 & 1.538570 \\
\hline $\mathrm{H}$ & -2.521649 & -1.648283 & 0.862339 \\
\hline $\mathrm{H}$ & -3.006583 & -1.492636 & 2.576506 \\
\hline $\mathrm{H}$ & -5.654177 & 2.941070 & 3.885604 \\
\hline $\mathrm{H}$ & -4.493409 & 4.225936 & 3.474277 \\
\hline $\mathrm{C}$ & -4.596141 & 3.139475 & 3.631040 \\
\hline $\mathrm{H}$ & -3.996887 & 2.860939 & 4.515113 \\
\hline C & 0.917272 & 0.680033 & -4.907930 \\
\hline C & 0.482995 & 1.302047 & -6.110936 \\
\hline C & 1.436363 & 1.539927 & -7.120523 \\
\hline $\mathrm{H}$ & 1.111043 & 2.022550 & -8.049369 \\
\hline $\mathrm{C}$ & 2.781538 & 1.183807 & -6.949948 \\
\hline $\mathrm{H}$ & 3.507047 & 1.379368 & -7.746517 \\
\hline C & 3.196118 & 0.587281 & -5.750288 \\
\hline $\mathrm{H}$ & 4.247281 & 0.311236 & -5.608116 \\
\hline C & 2.280243 & 0.331412 & -4.712868 \\
\hline C & 2.726114 & -0.320860 & -3.425394 \\
\hline $\mathrm{H}$ & 2.476853 & 0.301831 & -2.545821 \\
\hline C & -0.954399 & 1.739055 & -6.298566 \\
\hline $\mathrm{H}$ & -1.316938 & 2.318231 & -5.430180 \\
\hline C & 5.584952 & -0.200299 & 0.965131 \\
\hline $\mathrm{H}$ & 4.757029 & 1.187553 & -0.469411 \\
\hline O & 3.156450 & -0.131050 & 0.744749 \\
\hline C & 5.675801 & 0.774390 & -0.044306 \\
\hline $\mathrm{H}$ & 6.988112 & 1.970413 & -1.287284 \\
\hline C & 6.932381 & 1.210096 & -0.500457 \\
\hline $\mathrm{H}$ & 9.087743 & 1.018119 & -0.308284 \\
\hline C & 8.109527 & 0.677688 & 0.047543 \\
\hline $\mathrm{H}$ & 8.936863 & -0.721544 & 1.490500 \\
\hline C & 8.024433 & -0.297457 & 1.057333 \\
\hline $\mathrm{H}$ & 6.711546 & -1.496049 & 2.297681 \\
\hline C & 6.771211 & -0.733540 & 1.511249 \\
\hline C & 4.248289 & -0.683259 & 1.500229 \\
\hline $\mathrm{H}$ & 4.146250 & -0.384263 & 2.562778 \\
\hline $\mathrm{C}$ & 1.588504 & -2.567338 & 0.294176 \\
\hline $\mathrm{Si}$ & 1.607599 & -0.767380 & 0.817526 \\
\hline $\mathrm{C}$ & 0.615026 & -3.472704 & 0.779630 \\
\hline $\mathrm{H}$ & 1.515875 & 1.060024 & 3.209782 \\
\hline C & 0.595398 & -4.808819 & 0.348757 \\
\hline $\mathrm{H}$ & 4.218806 & -1.791445 & 1.476411 \\
\hline C & 1.551320 & -5.264947 & -0.574527 \\
\hline $\mathrm{H}$ & -0.129245 & -3.130524 & 1.506323 \\
\hline C & 2.528953 & -4.382724 & -1.064226 \\
\hline $\mathrm{H}$ & -0.165037 & -5.495705 & 0.735805 \\
\hline $\mathrm{C}$ & 2.545933 & -3.046155 & -0.633444 \\
\hline $\mathrm{H}$ & 1.536213 & -6.307569 & -0.909996 \\
\hline $\mathrm{H}$ & 3.280447 & -4.736385 & -1.778388 \\
\hline $\mathrm{H}$ & 3.318032 & -2.368670 & -1.016345 \\
\hline C & -0.047140 & 0.151841 & 4.385473 \\
\hline $\mathrm{H}$ & 0.374555 & -1.967142 & 4.338621 \\
\hline O & 0.919660 & -0.749214 & 2.341387 \\
\hline C & -0.067929 & -1.150579 & 4.916017 \\
\hline $\mathrm{H}$ & -0.662483 & -2.417285 & 6.571631 \\
\hline $\mathrm{C}$ & -0.651099 & -1.397472 & 6.171311 \\
\hline $\mathrm{H}$ & -1.668210 & -0.539371 & 7.888572 \\
\hline $\mathrm{C}$ & -1.214685 & -0.345987 & 6.910578 \\
\hline $\mathrm{H}$ & -1.629132 & 1.786925 & 6.955727 \\
\hline $\mathrm{C}$ & -1.192973 & 0.958818 & 6.386412 \\
\hline $\mathrm{H}$ & -0.601445 & 2.223410 & 4.726068 \\
\hline C & -0.615342 & 1.205161 & 5.132078 \\
\hline $\mathrm{C}$ & 0.596786 & 0.462912 & 3.046072 \\
\hline $\mathrm{H}$ & -0.087061 & 1.087989 & 2.438396 \\
\hline $\mathrm{H}$ & 0.196265 & 5.012283 & -1.969472 \\
\hline $\mathrm{C}$ & 1.053346 & 4.271325 & 0.509387 \\
\hline $\mathrm{Si}$ & 0.877986 & 3.810217 & -1.340426 \\
\hline
\end{tabular}

$\begin{array}{rrr}2.283279 & 4.115592 & 1.195103 \\ 2.294900 & 3.915128 & -1.871523 \\ 2.411987 & 4.447810 & 2.554761 \\ 0.854040 & 0.159414 & -0.100002 \\ 1.308928 & 4.949202 & 3.266359 \\ 3.161320 & 3.740754 & 0.655654 \\ 0.077274 & 5.110809 & 2.607903 \\ 3.378823 & 4.324826 & 3.056213 \\ -0.045773 & 4.772261 & 1.250230 \\ 1.409543 & 5.217773 & 4.323669 \\ -0.787573 & 5.508443 & 3.151160 \\ -1.015468 & 4.912139 & 0.756503\end{array}$

\section{C'}

$-0.687580$

$-2.739769$

$-0.616014$

$-4.112983$

$-2.960451$

$-4.339985$

$-4.807141$

$-4.965433$

$-6.028938$

$-3.953110$

$-1.846640$

$-2.014279$

$-4.701023$

$-4.851029$

$-5.294806$

$-4.447085$

$-3.874923$

$-3.562997$

$-3.699371$

$-3.103060$

$-2.042922$

$-3.132377$

$-3.637128$

2. 451581

0.680090

$-1.386571$

$-0.195381$

$-6.157500$

$-5.158679$

$-4.471480$

$-5.230953$

$-5.068476$

$-5.278852$

$-4.622981$

$-3.657125$

0.488806

0.535563

1.686278

1.733113

2.761225

3. 645089

2. 703191

3. 539238

1.580370

1.517469

1. 365437

$-0.583385$

$-1.057497$

4. 630002

4.192626

2. 333001

4.967305

6.526190

6.278504

8.287870

7.266865

7.699205

6.935971

5.376359

5.628050

3.225906

2. 857971
$-0.340351$

$-0.415061$

$-0.161613$

$-0.683021$

$-0.407573$

$-0.600699$

$-0.631541$

$-0.741445$

$-0.889327$

$-0.624126$

$-0.254213$

$-0.234251$

$-1.826204$

$-1.853668$

$-2.743759$

$-0.785643$

0.339892

1.193432

0.413379

1. 672575

1. 528701

2. 500141

1.989297

$-2.638793$

$-2.720380$

1. 994768

3. 251722

$-2.867324$

$-3.026581$

$-3.255635$

$-3.918568$

$-1.787687$

$-0.017208$

$-0.833182$

$-0.715395$

0.090469

1. 250479

1. 462098

2. 358595

0.564514

0.751831

$-0.569767$

$-1.278322$

$-0.822735$

$-2.058789$

$-1.801430$

2. 272478

2. 404124

$-0.679987$

$-0.010322$

$-0.903168$

$-0.103901$

0.788713

0.341443

0.563007

0.215654

$-0.461464$

$-0.359509$

$-1.249809$

$-0.802656$

$-1.182091$

$-0.710160$
$-1.803222$

$-2.237398$

$-3.992312$

$-0.163898$

$-3.616330$

$-3.885621$

$-4.871551$

$-2.637284$

$-2.446602$

$-1.639115$

$-4.483862$

$-5.571484$

0.448072

1. 848921

2. 312304

2. 667693

2.043659

2.658848

0.651136

0.054855

$-0.230919$

0.782665

$-0.856891$

$-4.025911$

$-4.240165$

$-6.422312$

$-6.041386$

$-0.802655$

$-0.356608$

$-1.187451$

0.287657

4. 494772

4. 524429

4.169843

4. 693506

$-4.855609$

$-5.681375$

$-6.468579$

$-7.097775$

$-6.451069$

$-7.069768$

$-5.627392$

$-5.606890$

$-4.819941$

$-3.953095$

$-2.885669$

$-5.715661$

$-4.728682$

0.768286

$-1.236125$

$-0.018602$

$-0.469845$

$-1.684022$

$-0.714801$

0.082389

0.273694

2. 293456

1. 514001

2. 727983

1. 758139

1.061722

1. 999369 


$\begin{array}{rr}\mathrm{C} & 0.476776 \\ \mathrm{Si} & 0.658535 \\ \mathrm{C} & -0.592021 \\ \mathrm{C} & -0.772934 \\ \mathrm{H} & 3.265000 \\ \mathrm{C} & 0.120508 \\ \mathrm{H} & -1.294374 \\ \mathrm{C} & 1.191104 \\ \mathrm{H} & -1.607142 \\ \mathrm{C} & 1.360780 \\ \mathrm{H} & -0.015439 \\ \mathrm{H} & 1.895411 \\ \mathrm{H} & 2.202961 \\ \mathrm{H} & 0.268357 \\ \mathrm{H} & 0.418585 \\ \mathrm{C} & 1.278362 \\ \mathrm{Si} & 1.149999 \\ \mathrm{C} & 2.195001 \\ \mathrm{H} & 2.499984 \\ \mathrm{C} & 2.277401 \\ \mathrm{H} & 0.378378 \\ \mathrm{C} & 1.446873 \\ \mathrm{H} & 2.855408 \\ \mathrm{C} & 0.534802 \\ \mathrm{H} & 2.993632 \\ \mathrm{C} & 0.452795 \\ \mathrm{H} & 1.513615 \\ \mathrm{H} & -0.110834 \\ \mathrm{H} & -0.258777\end{array}$

TS C'D'

\begin{tabular}{|c|c|c|c|}
\hline $\mathrm{Mn}$ & -0.658702 & 0.030678 & -1.891617 \\
\hline $\mathrm{N}$ & -2.655330 & -0.330246 & -2.185398 \\
\hline $\mathrm{N}$ & -0.624593 & -0.129919 & -4.041177 \\
\hline $\mathrm{C}$ & -3.932172 & -0.427723 & -0.033471 \\
\hline $\mathrm{C}$ & -2.922351 & -0.529837 & -3.544176 \\
\hline C & -4.286272 & -0.877843 & -3.719777 \\
\hline $\mathrm{H}$ & -4.781341 & -1.082243 & -4.670545 \\
\hline C & -4.851996 & -0.902203 & -2.436265 \\
\hline $\mathrm{H}$ & -5.889611 & -1.117158 & -2.178147 \\
\hline $\mathrm{C}$ & -3.824621 & -0.559635 & -1.508868 \\
\hline C & -1.857912 & -0.387315 & -4.469660 \\
\hline $\mathrm{H}$ & -2.057053 & -0.506405 & -5.544805 \\
\hline C & -4.339966 & -1.538810 & 0.757610 \\
\hline C & -4.432413 & -1.388192 & 2.155824 \\
\hline $\mathrm{H}$ & -4.736815 & -2.252874 & 2.758892 \\
\hline C & -4.145598 & -0.171996 & 2.798997 \\
\hline $\mathrm{C}$ & -3.764318 & 0.921419 & 1.997405 \\
\hline $\mathrm{H}$ & -3.557899 & 1.889068 & 2.471887 \\
\hline $\mathrm{C}$ & -3.654275 & 0.819966 & 0.599501 \\
\hline $\mathrm{C}$ & -3.289714 & 2.051517 & -0.202880 \\
\hline $\mathrm{H}$ & -2.257754 & 1.996309 & -0.600143 \\
\hline $\mathrm{H}$ & -3.353233 & 2.957538 & 0.421217 \\
\hline $\mathrm{H}$ & -3.953226 & 2.180666 & -1.075499 \\
\hline $\mathrm{H}$ & 2.646465 & -2.330786 & -3.980413 \\
\hline $\mathrm{H}$ & 0.876322 & -2.567559 & -4.040028 \\
\hline $\mathrm{H}$ & -1.725041 & 1.689709 & -6.535755 \\
\hline $\mathrm{H}$ & -0.593923 & 3.055290 & -6.483136 \\
\hline $\mathrm{H}$ & -5.683799 & -2.892188 & -0.302378 \\
\hline C & -4.672074 & -2.883896 & 0.142543 \\
\hline $\mathrm{H}$ & -3.970035 & -3.158026 & -0.662050 \\
\hline $\mathrm{H}$ & -4.649147 & -3.677245 & 0.908225 \\
\hline $\mathrm{H}$ & -4.677765 & -0.934497 & 4.765206 \\
\hline $\mathrm{H}$ & -4.861040 & 0.832336 & 4.591061 \\
\hline $\mathrm{C}$ & -4.239990 & -0.034533 & 4.302975 \\
\hline $\mathrm{H}$ & -3.243664 & 0.123321 & 4.755416 \\
\hline C & 0.414964 & 0.114213 & -4.988225 \\
\hline C & 0.319246 & 1.177641 & -5.930649 \\
\hline $\mathrm{C}$ & 1.394521 & 1.375937 & -6.820550 \\
\hline $\mathrm{H}$ & 1.331473 & 2.196789 & -7.544288 \\
\hline $\mathrm{C}$ & 2.533927 & 0.561665 & -6.786161 \\
\hline $\mathrm{H}$ & 3.356540 & 0.736246 & -7.487530 \\
\hline C & 2.621873 & -0.467542 & -5.837086 \\
\hline $\mathrm{H}$ & 3.512471 & -1.105282 & -5.797474 \\
\hline C & 1.577567 & -0.702614 & -4.925372 \\
\hline C & 1.674895 & -1.815667 & -3.909576 \\
\hline
\end{tabular}

$\begin{array}{rr}-3.140062 & 0.412271 \\ -1.251042 & 0.161987 \\ -3.675081 & 1.172573 \\ -5.061824 & 1.304871 \\ -2.274740 & 1.260413 \\ -5.951674 & 0.683741 \\ -2.997566 & 1.675162 \\ -5.444549 & -0.072008 \\ -5.449370 & 1.900874 \\ -4.056395 & -0.209176 \\ -7.033495 & 0.790465 \\ -6.131686 & -0.555049 \\ -3.677573 & -0.801793 \\ -0.695178 & 1.527141 \\ 4.261993 & -2.458205 \\ 4.007425 & 0.167631 \\ 3.299280 & -1.573275 \\ 3.477461 & 1.108121 \\ 3.007818 & -2.149759 \\ 4.003340 & 2.406998 \\ 1.997728 & -1.591263 \\ 5.070527 & 2.789350 \\ 2.649019 & 0.826717 \\ 5.611451 & 1.868692 \\ 3.581669 & 3.120333 \\ 5.084639 & 0.569232 \\ 5.481810 & 3.802331 \\ 6.447005 & 2.160136 \\ 5.522926 & -0.140532\end{array}$

0.030678

$-0.129919$

$-0.427723$

$-0.877843$

1.082243

$-0.559635$

1.538810

1.388192

0.171996

0.819966

051517

2.957538

1. 689709

3.055290

$-2.883896$

$-3.158026$

$-0.934497$

.832336

0.123321

114213

1. 375937

$-1.815667$
1.574013

$-0.870677$

$-1.237714$

4.672753

4. 449059

2. 403204

5.155298

6.886445

6.524890

8.492370

7.425807

7.642943

6.948855

5.215417

5. 582160

3.204386

2. 855065

0.595925

0.755450

$-0.544281$

$-0.690479$

3.085280

0.303639

$-1.322226$

1. 444944

$-1.577076$

1. 585971

0.191588

2. 227882

2. 483034

0.068467

$-0.193227$

0.838226

0.659515

1. 770215

2.009383

1.888834

0.538236

1.073442

2. 410702

0.141410

2. 620759

0.026930

1.166009

$-0.494227$

$-0.697622$

\section{D'}

$-0.659898$

$-2.508581$

$-0.804051$

$-3.579070$

$-2.820480$

$-4.059978$

$-4.557788$

$-4.507665$

$-5.437237$

$-3.534501$

$-1.930031$

$-2.182201$

$-3.712092$

$-3.745492$

$-3.833603$

$-3.665204$

$-3.561545$

$-3.519686$

$-3.518532$

$-3.454749$

$-2.427793$

$-3.764170$

$-4.102764$

2. 917943

1. 270189

$-2.576757$

$-1.864698$

$-4.854265$
$-1.427995$

2. 114913

2. 365490

$-0.743344$

$-0.040471$

$-0.573124$

$-0.229994$

0.445732

0.042441

0.015709

$-0.197813$

$-0.898243$

$-0.711090$

$-1.375498$

$-0.979449$

$-1.073192$

$-0.637692$

$-2.868050$

$-0.989653$

$-3.551056$

$-4.935967$

$-2.173688$

$-5.670820$

$-2.997071$

$-5.013274$

$-5.444933$

$-3.626664$

$-6.752047$

$-5.582559$

$-3.127398$

$-0.635346$

3. 293329

3. 325670

2. 408584

2.898789

2. 320741

3. 568841

0.581324

4.678589

2. 031629

5.115913

3. 224430

4. 447046

5.201182

5.983645

4.809664

0.808751

$-0.182863$

0.077747

0.013917

$-0.780408$

$-1.463134$

$-2.009590$

$-1.278943$

$-1.645617$

$-0.482089$

$-0.607488$

$-1.051621$

$-0.901774$

$-0.406804$

$-1.121844$

0.965982

1.858494

2. 937142

1. 414460

2. 445268

2.559343

3. 435486

2. 175590

$-1.228563$

$-1.880949$

0.966435

2. 508598

$-2.701158$
$-2.877028$

$-5.978710$

$-4.968974$

1. 218237

$-0.812159$

0.339429

0.001014

$-1.114702$

$-0.162355$

0.758700

0.886624

2. 932633

2. 105991

3. 225547

2. 270499

1. 422630

2. 381778

0.047491

0.245397

0.541295

0.364690

1. 510382

$-0.304695$

1. 080669

$-0.795777$

0.758507

$-0.627521$

$-0.438991$

$-1.309099$

$-1.012040$

1. 542205

$-2.353712$

0.176275

$-1.471305$

1. 155975

$-2.133096$

2. 383747

$-0.776683$

2. 665067

0.955518

1. 709300

3. 122847

0.479403

3. 623323

1. 918513

$-0.259495$

$-2.295495$

$-2.171486$

$-4.333775$

0.085331

$-3.396946$

$-3.302808$

$-4.105781$

$-1.986439$

$-1.549727$

$-1.313444$

$-4.489467$

$-5.464830$

1. 167788

2. 486195

3. 313907

2. 773826

1. 689673

1.885710

0.356770

$-0.752347$

$-1.153884$

$-0.379264$

$-1.603203$

$-4.328782$

$-4.100386$

$-6.833959$

$-7.349444$

0.705393 


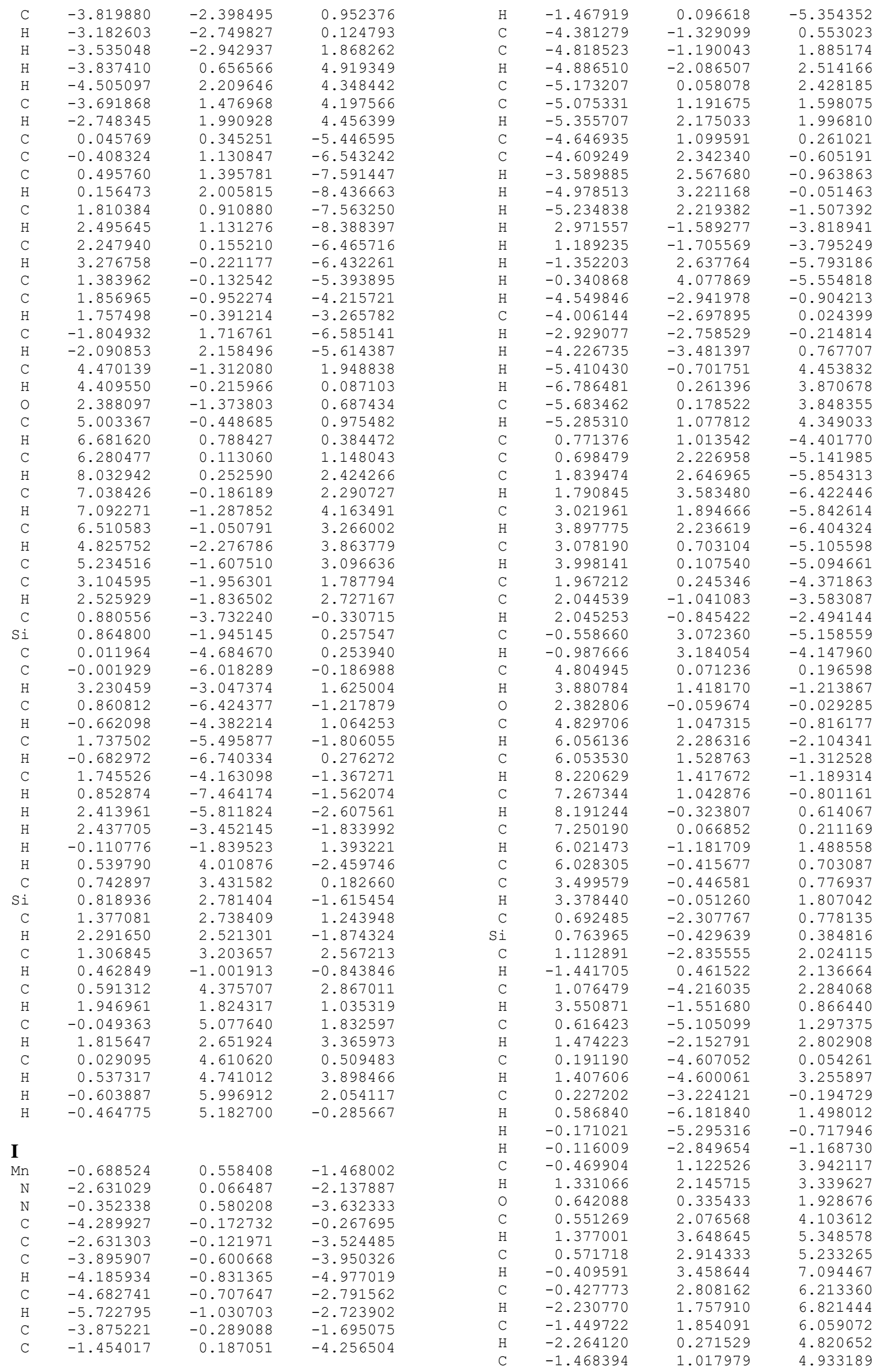




$\begin{array}{lr}\mathrm{C} & -0.529439 \\ \mathrm{H} & -0.668087 \\ \mathrm{C} & -0.126079 \\ \mathrm{H} & 0.659967 \\ \mathrm{O} & -0.523669 \\ \mathrm{C} & 0.523572 \\ \mathrm{H} & 1.487544 \\ \mathrm{C} & 0.982608 \\ \mathrm{H} & 1.159526 \\ \mathrm{C} & 0.797398 \\ \mathrm{H} & 0.018507 \\ \mathrm{C} & 0.154794 \\ \mathrm{H} & -0.800198 \\ \mathrm{C} & -0.306451 \\ \mathrm{C} & -0.618269 \\ \mathrm{H} & -1.098371\end{array}$

0.212241
-0.836031
4.724331
4.861597
2.682646
5.387918
7.212434
6.695148
8.374849
7.349573
7.210340
6.696697
4.859894
5.385930
3.358836
2.916160

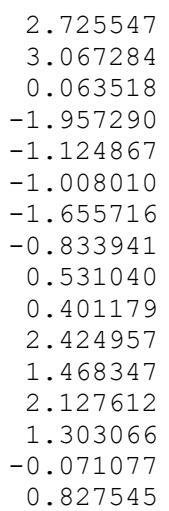

\section{TS IJ}

$\begin{array}{cc}\text { Mn } & -1.023334 \\ \text { N } & -2.603587 \\ \text { N } & -0.419887 \\ \text { C } & -4.347938 \\ \text { C } & -2.425568 \\ \text { C } & -3.510470 \\ \text { H } & -3.647491 \\ \text { C } & -4.362078\end{array}$

C $\quad-4.362078$

$\mathrm{H} \quad-5.311235$

$-3.781282$

$\begin{array}{ll}\mathrm{C} & -1.297403 \\ \mathrm{H} & -1.187543\end{array}$

C $\quad-4.628781$

C $\quad-5.199992$

$\mathrm{H} \quad-5.415093$

C $\quad-5.507248$

C $\quad-5.231098$

$\mathrm{H} \quad-5.485674$

$\begin{array}{ll}\text { C } & -4.663569 \\ \text { C } & -4.460434\end{array}$

H $\quad-3.390179$

$\mathrm{H} \quad-4.955767$

$\mathrm{H} \quad-4.867233$

2. 480103

0.875787

$-1.123298$

0.162690

$-5.073524$

$-4.330731$

$-3.342691$

$-4.352973$

$-6.612003$

$-6.830985$

$-6.101946$

$-5.320929$

0.704572

0.900093

2. 017054

2. 158911

2. 936387

3. 802689

2. 740852

3. 463075

1. 633076

1.490161

1.019904

$-0.094200$

$-0.112162$

4. 729802

3. 591024

2. 328885

4.591973

5.604571

5.728770

7.898369

7.013606

8.155847

7. 157589
0.823707

$-0.424319$

0.086705

$-0.247805$

$-1.132373$

$-2.024351$

$-2.696961$

$-1.853338$

$-2.358551$

$-0.852919$

$-0.828486$

$-1.331019$

$-1.053520$

$-0.455201$

$-1.085648$

0.916513

1.695293

2. 762716

1. 143750

2. 033189

2. 230830

3. 007587

1. 569507

$-2.537583$

$-2.607610$

2. 578090

3. 811147

$-3.113956$

$-2.539204$

$-2.766955$

$-2.927221$

0.790153

2. 329856

1. 540980

2. 012882

0.405965

1. 776188

2. 129393

3. 181247

1. 160705

1. 451721

$-0.180458$

$-0.940233$

$-0.587918$

$-2.051818$

$-2.192058$

2. 831420

2.958103

1.068609

1.541625

1. 374321

1. 396448

1. 804969

1.547023

1. 488017

1. 369039

0.905469

1. 041405
$-1.563241$

$-1.930437$

$-3.539482$

$-0.147250$

$-3.121495$

$-3.316243$

$-4.164703$

$-2.213016$

$-1.380415$

$-3.930828$

0.991762

2. 132418

3. 004485

2.185077

1. 044992

1.051643

$-0.118195$

$-1.329534$

$-1.534161$

$-1.185908$

$-2.245149$

$-4.393525$

$-5.144876$

$-4.574522$

$-4.698329$

0.428132

1. 010077

0.576409

2. 041876

4.054965

3. 176138

3.428766

4.053987

$-4.352783$

$-4.694152$

$-5.471410$

$-5.744807$

$-5.901561$

$-6.504857$

$-5.549339$

$-5.870147$

$-4.777974$

$-4.412696$

$-3.425813$

$-4.263443$

$-3.163826$

$-0.214393$

$-1.991827$

0.084763

$-1.575212$

$-3.445642$

$-2.388451$

$-2.487108$

$-1.852555$

$-0.062266$

$-0.492586$
$-2.030117$

$-4.903555$
6.142541

6.023800

3. 528227

3. 697161

0.831541

0.791326

0.189062

$-1.219852$

0.191742

3. 418846

0.842002

$-0.296563$

1. 495767

$-0.303481$

1. 482031

0.846844

2. 021788

1. 998901

$-0.698530$

1. 302427

0.436066

0.468241

1. 477609

0.563700

$-0.430242$

$-0.506695$

$-2.507777$

$-1.672185$

$-2.670551$

$-1.764494$

$-0.836818$

$-1.580965$

0.117867

2. 080959

$-0.048322$

1.534124

3. 289493

2. 205645

2.050644

1. 513686

$-0.430454$

0.119592

$-1.651769$

$-0.568715$

$-0.635502$

$-1.709884$

J

$-1.414286$

$-3.446362$

$-1.831120$

$-4.314803$

$-3.927937$

$-5.266051$

$-5.898993$

$-5.600533$

$-6.555463$

$-4.459831$

$-3.064368$

$-3.435536$

$-4.309874$

$-4.159352$

$-4.148293$

$-4.024152$

$-4.057982$

$-3.984196$

$-4.199596$

$-4.269102$

$-3.347727$

$-4.410969$

$-5.101039$

$-0.087210$

$-1.796432$

$-2.276061$

$-0.763249$

$-5.504113$

0.646556

0.895521

0.867156

1.390193

$-1.034544$

0.864364

$-1.776737$

2. 488716

$-3.180812$

$-0.211606$

$-3.878708$

$-1.256983$

$-3.161489$

$-3.732015$

$-1.758751$

$-4.973852$

$-3.697356$

$-1.215954$

1. 023137

1.817474

1. 423231

1. 317293

1.193555

0.962497

0.038953

0.315013

$-0.495600$

0.018010

0.121106

0.365825

1. 450074

0.807010

4.505974

3. 763709

2. 240393

4.618207

5.851521

5.790384

7.805108

6.890614

7. 640569

6.795431

5.567400

5.630992 


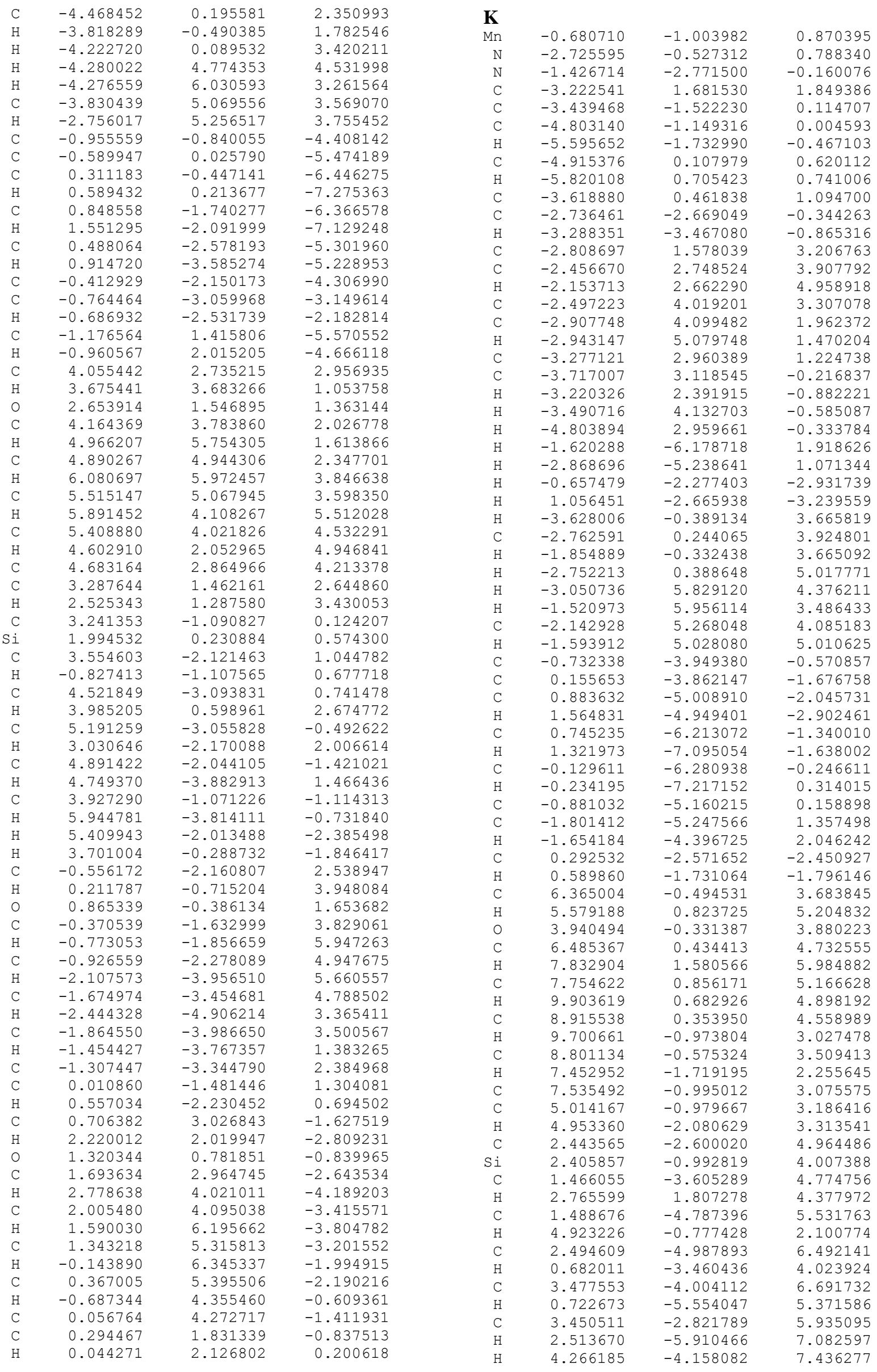




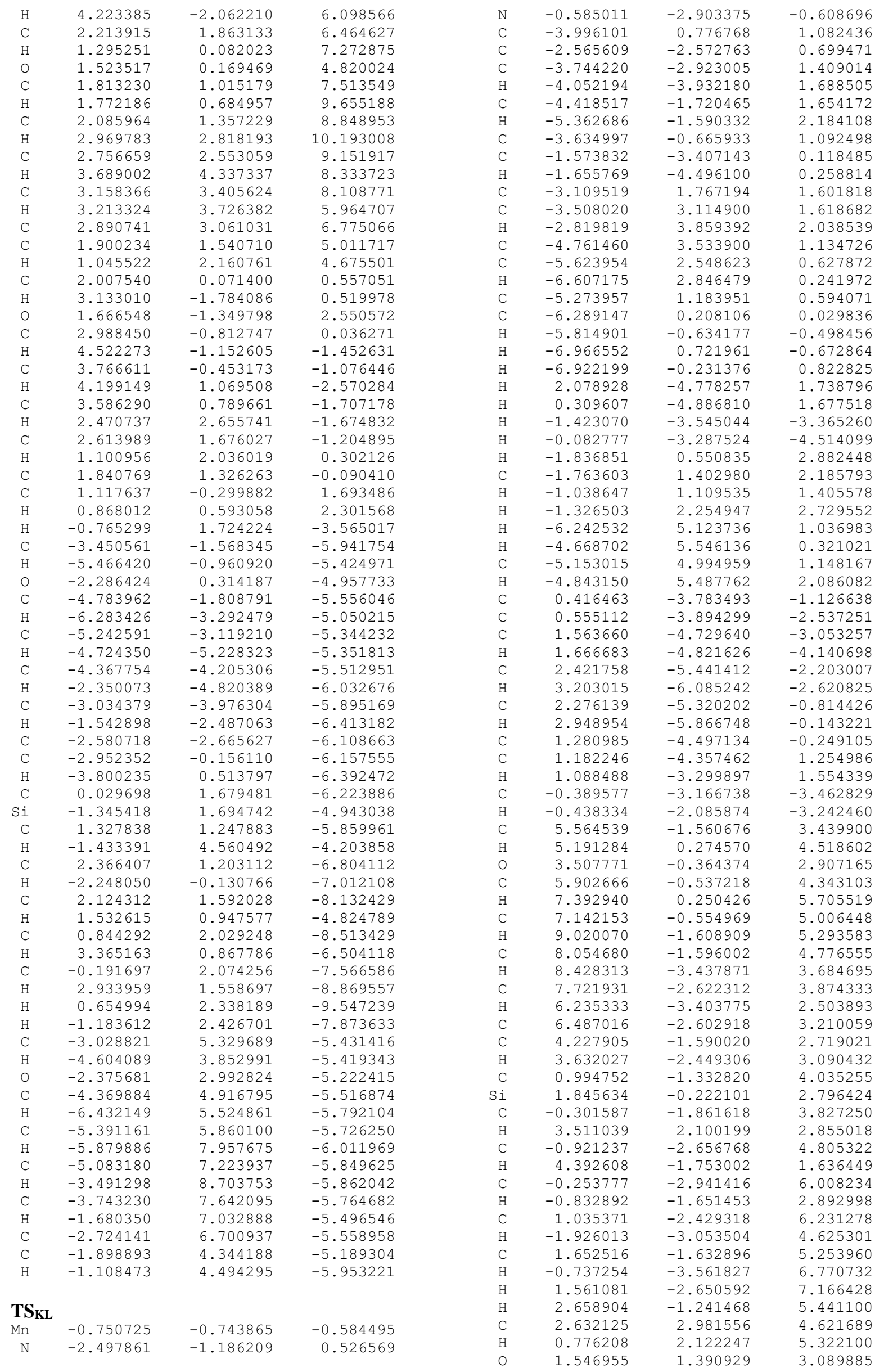




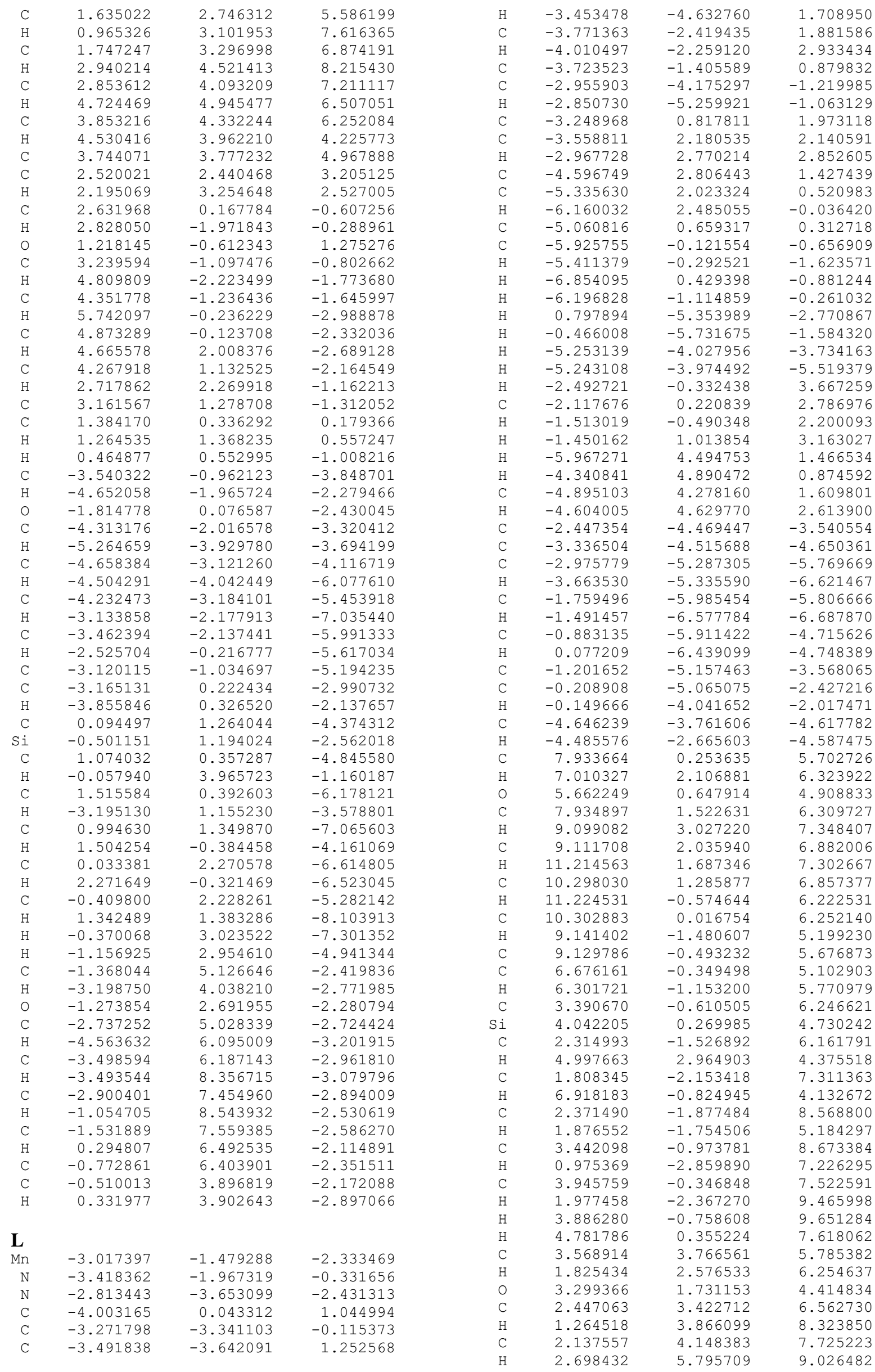




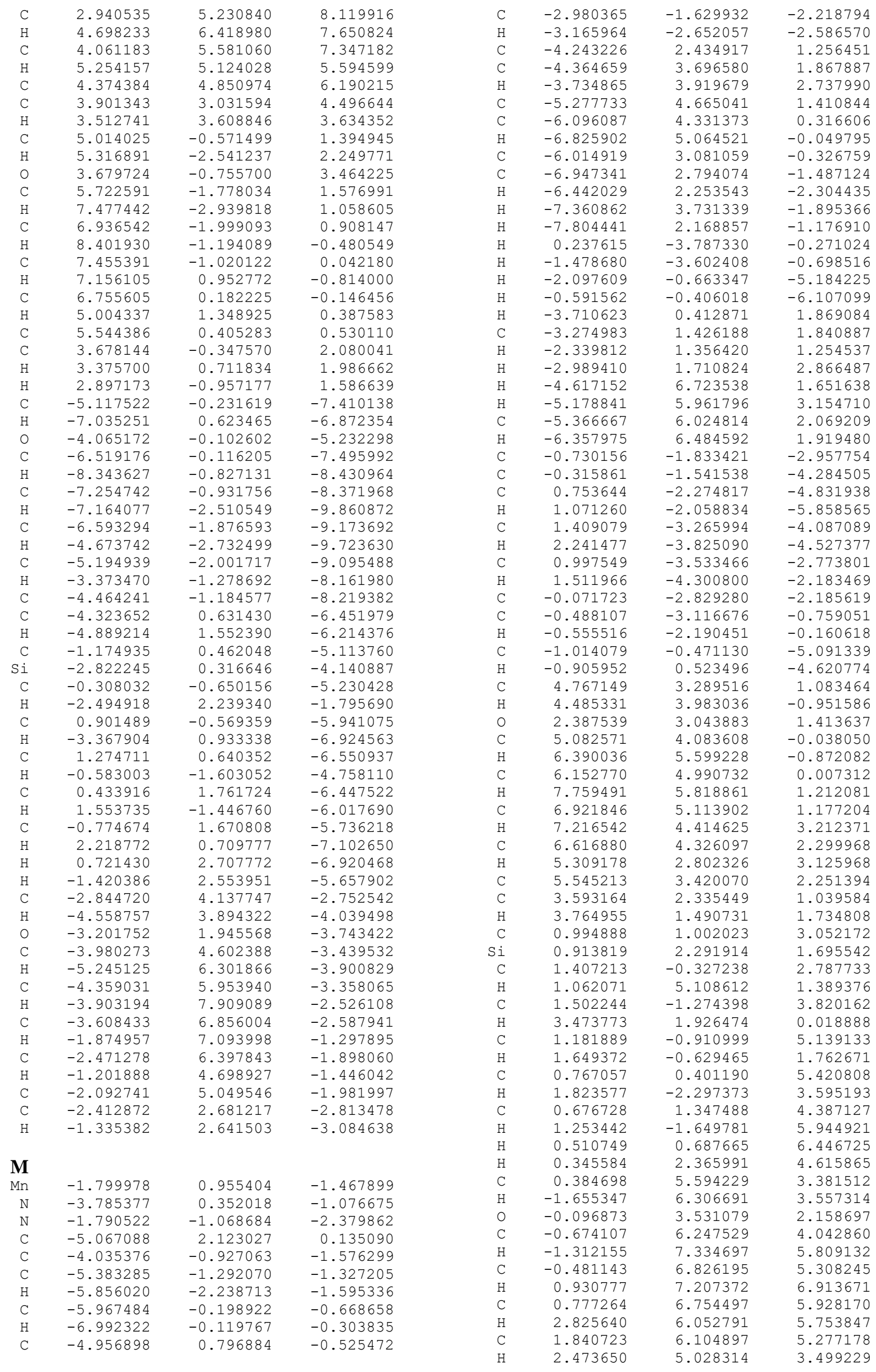




$\begin{array}{rr}\mathrm{C} & 1.645336 \\ \mathrm{C} & 0.174660 \\ \mathrm{H} & -0.700671 \\ \mathrm{C} & 0.768904 \\ \mathrm{H} & 1.941368 \\ \mathrm{O} & 0.311926 \\ \mathrm{C} & 1.823788 \\ \mathrm{H} & 3.510948 \\ \mathrm{C} & 2.704065 \\ \mathrm{H} & 3.245811 \\ \mathrm{C} & 2.557847 \\ \mathrm{H} & 1.400593 \\ \mathrm{C} & 1.518912 \\ \mathrm{H} & -0.146267 \\ \mathrm{C} & 0.642873 \\ \mathrm{C} & -0.221850 \\ \mathrm{H} & -0.624171 \\ \mathrm{H} & -4.764395 \\ \mathrm{C} & -3.095091 \\ \mathrm{Si} & -3.318460 \\ \mathrm{C} & -2.503229 \\ \mathrm{H} & -2.665235 \\ \mathrm{C} & -2.340957 \\ \mathrm{H} & -2.687330 \\ \mathrm{C} & -2.765750 \\ \mathrm{H} & -2.164103 \\ \mathrm{C} & -3.354011 \\ \mathrm{H} & -1.880998 \\ \mathrm{C} & -3.517300 \\ \mathrm{H} & -2.638252 \\ \mathrm{H} & -3.686196 \\ \mathrm{H} & -3.980512 \\ & \\ \mathrm{H} & \end{array}$

\section{TSMN}

\begin{tabular}{|c|c|c|c|}
\hline & & & \\
\hline Mn & -1.875088 & 1.316799 & -1.317614 \\
\hline $\mathrm{N}$ & -3.709166 & 0.546594 & -0.624957 \\
\hline $\mathrm{N}$ & -1.672729 & -0.712484 & -2.085122 \\
\hline C & -5.141371 & 2.168603 & 0.668403 \\
\hline C & -3.858501 & -0.758501 & -1.108747 \\
\hline C & -5.149236 & -1.253986 & -0.792410 \\
\hline $\mathrm{H}$ & -5.536473 & -2.244008 & -1.039842 \\
\hline C & -5.804906 & -0.220684 & -0.108685 \\
\hline $\mathrm{H}$ & -6.812655 & -0.236918 & 0.308656 \\
\hline C & -4.894439 & 0.872123 & -0.019368 \\
\hline C & -2.796061 & -1.369252 & -1.821708 \\
\hline $\mathrm{H}$ & -2.922825 & -2.406053 & -2.170481 \\
\hline C & -4.481719 & 2.460012 & 1.893875 \\
\hline C & -4.769426 & 3.669064 & 2.556530 \\
\hline $\mathrm{H}$ & -4.270877 & 3.877443 & 3.511490 \\
\hline C & -5.690313 & 4.600273 & 2.043810 \\
\hline C & -6.344219 & 4.285037 & 0.837788 \\
\hline $\mathrm{H}$ & -7.074996 & 4.990952 & 0.423174 \\
\hline C & -6.095740 & 3.086103 & 0.144719 \\
\hline C & -6.855340 & 2.795960 & -1.133709 \\
\hline $\mathrm{H}$ & -6.192686 & 2.419760 & -1.930712 \\
\hline $\mathrm{H}$ & -7.355704 & 3.705406 & -1.505518 \\
\hline $\mathrm{H}$ & -7.636024 & 2.028933 & -0.979794 \\
\hline $\mathrm{H}$ & 0.496638 & -3.529843 & -0.252099 \\
\hline $\mathrm{H}$ & -1.224672 & -3.539599 & -0.682733 \\
\hline $\mathrm{H}$ & -2.039007 & -0.130222 & -4.905430 \\
\hline $\mathrm{H}$ & -0.524790 & 0.340744 & -5.728291 \\
\hline $\mathrm{H}$ & -3.935885 & 0.451311 & 2.527142 \\
\hline C & -3.519707 & 1.473987 & 2.522528 \\
\hline $\mathrm{H}$ & -2.561546 & 1.422775 & 1.977127 \\
\hline $\mathrm{H}$ & -3.292227 & 1.758683 & 3.562533 \\
\hline $\mathrm{H}$ & -5.435201 & 6.748055 & 2.277838 \\
\hline $\mathrm{H}$ & -5.640733 & 5.868113 & 3.811154 \\
\hline C & -5.967263 & 5.905623 & 2.758480 \\
\hline $\mathrm{H}$ & -7.042036 & 6.156710 & 2.742934 \\
\hline C & -0.612112 & -1.408763 & -2.743979 \\
\hline C & -0.233799 & -0.995035 & -4.050021 \\
\hline $\mathrm{C}$ & 0.826667 & -1.663468 & -4.689642 \\
\hline $\mathrm{H}$ & 1.114126 & -1.351613 & -5.700214 \\
\hline C & 1.508418 & -2.712779 & -4.056274 \\
\hline $\mathrm{H}$ & 2.334704 & -3.219880 & -4.565671 \\
\hline C & 1.129268 & -3.106076 & -2.765812 \\
\hline
\end{tabular}

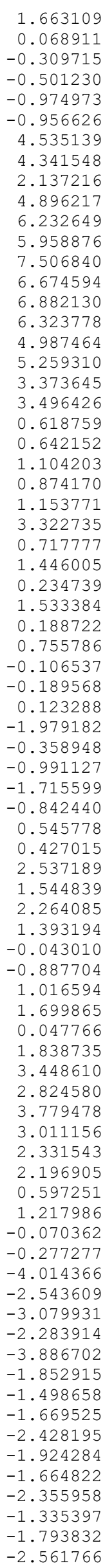

\subsection{9}

5.529452

5.399184

2. 348072

0.589908

1.475694

1.421372

0.826684

1.556570

2. 718342

2.614706

4.386778

3.544749

4.165779

3.418230

2.187815

3.171121

2. 813407

5.081291

3.209104

5.740070

2.640061

7.135561

2.575206

7.897069

5.158725

7.260161

7.626861

5.866198

8.984921

7.850593

5.388528
$-3.921259$

$-2.471705$

$-2.919771$

$-2.064255$

0.121391

1.052375

3. 397564

4. 207926

3.136423

4.255472

5.818956

5.160392

5.919722

5.216646

4.401213

4.364861

2. 792980

3. 461103

2.441019

1. 577156

0.936324

2. 423543

$-0.328927$

5.255231

$-1.408108$

2. 066504

$-1.241916$

$-0.477861$

0.006020

$-2.379201$

1.085828

$-2.084438$

0.139044

2.055416

5.427389

5.868038

3.606390

5.828404

6.495973

6.181995

6.420124

6.139547

5.715484

5.743107

5.082752

5.388625

5.023072

5.569342

2. 912568

0.883736

1.901588

1.778267

0.907476

1.795214

2. 941672

2. 932750

4.958806

4.061916

4. 945525

4.055888

2. 921434

3. 911617

3. 909541

4. 550762

3. 230714

4.220176

2. 212640

5.191099

2. 880997

6.521662

3.189400

6.873585

4.909637

5.899876

7.280564

7. 910784

6.198386
$-2.263289$

$-2.087441$

$-0.691375$

$-0.021663$

$-4.746729$

$-4.150622$

0.904677

$-1.098354$

1.064003

$-0.154525$

$-0.839434$

$-0.008177$

1. 313132

1.200244

3.202585

2. 260958

2. 937961

2. 112241

0.750518

1.430676

2. 364898

1.238584

1.951759

1. 378142

2. 849343

$-0.288167$

4. 174413

0.921425

4. 601744

2. 514583

3.705953

4.873363

5.633941

4.045823

3. 395305

3.687237

1.826379

4.159946

6.092454

5.510378

7.163748

6.111652

5.820728

5.357833

3. 417580

4.007983

1.945574

1. 490527

$-2.328292$

$-1.943213$

$-0.284108$

$-2.557679$

$-3.708890$

$-3.557066$

$-5.141083$

$-4.361081$

$-4.763569$

$-4.148803$

$-2.990367$

$-3.147734$

$-1.336418$

$-0.898724$

$-2.068677$

$-4.287383$

$-3.033062$

$-5.640963$

$-3.800211$

$-6.558527$

$-2.154007$

$-6.144912$

$-5.986937$

$-4.808222$

$-7.600791$

$-3.892926$

$-6.860626$

$-4.479217$

$-2.857374$ 
N

\begin{tabular}{|c|c|c|c|}
\hline & & 1.001900 & -1.174014 \\
\hline $\mathrm{N}$ & -4.928966 & 0.478847 & -0.807754 \\
\hline $\mathrm{N}$ & -2.828349 & -0.325031 & -2.390745 \\
\hline C & -6.560762 & 1.887701 & 0.448802 \\
\hline C & -4.731301 & -0.879093 & -1.071189 \\
\hline $\mathrm{C}$ & -5.755727 & -1.642498 & -0.455775 \\
\hline $\mathrm{H}$ & -5.856476 & -2.728924 & -0.483785 \\
\hline C & -6.597293 & -0.721284 & 0.189260 \\
\hline $\mathrm{H}$ & -7.486503 & -0.942837 & 0.780793 \\
\hline C & -6.059616 & 0.577748 & -0.043610 \\
\hline C & -3.625169 & -1.254879 & -1.880043 \\
\hline $\mathrm{H}$ & -3.447129 & -2.320354 & -2.093155 \\
\hline C & -5.766058 & 2.669445 & 1.337283 \\
\hline C & -6.259829 & 3.906151 & 1.789917 \\
\hline $\mathrm{H}$ & -5.647342 & 4.495378 & 2.484006 \\
\hline C & -7.515402 & 4.401727 & 1.389519 \\
\hline C & -8.287480 & 3.610985 & 0.520216 \\
\hline $\mathrm{H}$ & -9.270598 & 3.975688 & 0.197162 \\
\hline C & -7.839440 & 2.363333 & 0.044431 \\
\hline C & -8.728359 & 1.568170 & -0.890834 \\
\hline $\mathrm{H}$ & -8.158634 & 1.123682 & -1.724028 \\
\hline $\mathrm{H}$ & -9.517515 & 2.209861 & -1.316737 \\
\hline $\mathrm{H}$ & -9.231313 & 0.733546 & -0.369422 \\
\hline $\mathrm{H}$ & 0.427031 & -1.978976 & -0.754768 \\
\hline $\mathrm{H}$ & -1.207260 & -2.658877 & -0.908673 \\
\hline $\mathrm{H}$ & -3.721907 & -0.156145 & -5.140219 \\
\hline $\mathrm{H}$ & -2.589708 & 0.756690 & -6.175166 \\
\hline $\mathrm{H}$ & -4.424322 & 1.110853 & 2.065302 \\
\hline C & -4.416283 & 2.188947 & 1.832325 \\
\hline $\mathrm{H}$ & -3.619661 & 2.347580 & 1.080008 \\
\hline $\mathrm{H}$ & -4.115279 & 2.738332 & 2.739548 \\
\hline $\mathrm{H}$ & -7.479983 & 6.575141 & 1.358996 \\
\hline $\mathrm{H}$ & -7.830848 & 5.880908 & 2.953748 \\
\hline C & -8.007182 & 5.749186 & 1.871881 \\
\hline $\mathrm{H}$ & -9.085715 & 5.878396 & 1.683172 \\
\hline C & -1.681800 & -0.679868 & -3.159221 \\
\hline C & -1.635842 & -0.261709 & -4.517686 \\
\hline C & -0.490446 & -0.562081 & -5.277000 \\
\hline $\mathrm{H}$ & -0.454636 & -0.250998 & -6.327368 \\
\hline C & 0.592634 & -1.249753 & -4.709135 \\
\hline $\mathrm{H}$ & 1.479215 & -1.473493 & -5.311683 \\
\hline C & 0.539886 & -1.639827 & -3.363624 \\
\hline $\mathrm{H}$ & 1.391258 & -2.161339 & -2.911284 \\
\hline C & -0.586897 & -1.363998 & -2.562761 \\
\hline C & -0.595517 & -1.758931 & -1.100924 \\
\hline $\mathrm{H}$ & -1.002815 & -0.954765 & -0.463953 \\
\hline C & -2.808410 & 0.465804 & -5.134965 \\
\hline $\mathrm{H}$ & -3.060087 & 1.388513 & -4.575269 \\
\hline C & 6.018517 & 3.508171 & 2.265028 \\
\hline $\mathrm{H}$ & 6.503013 & 4.286788 & 0.299404 \\
\hline 0 & 3.785011 & 2.986048 & 1.504738 \\
\hline C & 6.656229 & 4.400433 & 1.378673 \\
\hline $\mathrm{H}$ & 7.976095 & 6.107766 & 1.169377 \\
\hline $\mathrm{C}$ & 7.483431 & 5.422900 & 1.868010 \\
\hline $\mathrm{H}$ & 8.333527 & 6.359679 & 3.634049 \\
\hline C & 7.684536 & 5.564294 & 3.252073 \\
\hline $\mathrm{H}$ & 7.211706 & 4.783027 & 5.222648 \\
\hline C & 7.055122 & 4.679672 & 4.143479 \\
\hline $\mathrm{H}$ & 5.735328 & 2.967360 & 4.345526 \\
\hline C & 6.225853 & 3.659108 & 3.650561 \\
\hline C & 5.102767 & 2.426870 & 1.734888 \\
\hline $\mathrm{H}$ & 5.034371 & 1.596591 & 2.464103 \\
\hline C & 2.015817 & 0.687853 & 2.153244 \\
\hline $8 i$ & 2.490967 & 2.059829 & 0.973707 \\
\hline C & 2.608426 & -0.596426 & 2.080447 \\
\hline $\mathrm{H}$ & 2.401939 & 4.874246 & 0.669717 \\
\hline $\mathrm{C}$ & 2.269429 & -1.601686 & 3.000350 \\
\hline $\mathrm{H}$ & 5.500213 & 2.019902 & 0.785938 \\
\hline C & 1.326989 & -1.342111 & 4.009780 \\
\hline $\mathrm{H}$ & 3.335015 & -0.816695 & 1.290632 \\
\hline C & 0.725094 & -0.075507 & 4.095451 \\
\hline $\mathrm{H}$ & 2.738150 & -2.589282 & 2.927799 \\
\hline C & 1.068317 & 0.929267 & 3.176638 \\
\hline $\mathrm{H}$ & 1.060518 & -2.126455 & 4.726689 \\
\hline
\end{tabular}

-0.013529
0.590095
0.805317
-1.088077
1.229415
-0.480069
-1.980566
-0.981601
-0.590303
-0.201560
1.697642
1.082604
2.588149
1.583357
1.346368
0.765773
4.374628
4.987346
2.815475
5.304127
7.333915
6.621626
8.054289
7.026575
6.415107
6.106864
4.078558
4.790838
2.934558
2.444674
-1.607407
-4.174484
-2.992045
-5.520147
-3.086442
-6.405450
-5.372251
-5.969368
-5.884684
-4.641395
-7.438287
-3.758074
-289106
-16

0.129670

1. 912039

5.195820

5.787296

3.134266

5.771363

6.777268

6.328031

6.746210

6.310611

5. 729857

5.738107

4. 749679

5.186479

4.582057

4.939273

2.115493

0.164909

1. 280734

1.072214

0.378048

1. 195152

2. 455231

2. 360180

4. 317120

3. 403164

4. 102502

3.282193

1. 966978

2. 958521

4.412019

4. 888318

4.119057

5.193376

5.022008

5.715047

1. 359961

5. 939281

5.028319

5. 641722

5.948384

5. 125123

6. 345311

5. 818679

4. 911963

0.485617

0.382019

1.399775

$-0.568064$

0.839095

0.696989

0.971109

0.139043

$-0.108353$

$-0.041483$

1. 363045

1. 754772

$-1.969331$

$-2.434140$

$-3.513620$

$-1.558829$

$-0.180650$

0.513007

0.336129

1.849309

2.022803

2. 564081

2. 460125

$-1.156052$

0.208205

4. 834051

4.492048

$-3.860552$

$-2.977486$
4.878054

3.247760

2. 085439

1.209033

0.829838

2. 121408

3. 322657

3. 309965

5.404689

4. 477851

5. 358622

4. 452122

3. 235468

3. 263223

0.810127

$-0.058698$

$-2.191309$

$-1.474314$

$-0.471220$

$-1.999176$

$-2.309247$

$-2.467860$

$-3.508646$

$-3.142331$

$-3.859311$

$-3.340078$

$-3.011318$

$-2.862374$

$-1.732592$

$-1.697548$

$-2.802010$

$-3.554740$

$-2.259209$

$-3.228717$

$-1.044745$

$-4.186022$

$-2.468252$

$-5.503183$

$-2.206826$

$-5.851206$

$-3.902862$

$-4.887765$

$-6.251476$

$-6.873997$

$-5.177642$

$-0.953381$

$-1.504117$

$-2.938851$

0.337949

$-2.812643$

$-3.175546$

$-4.134133$

$-2.059102$

$-1.962192$

$-1.047415$

$-3.518048$

$-4.538324$

0.588066

1. 902900

2. 091114

2. 978963

2. 706827

3. 536055

1. 408709

1. 182796

0.092304

1.796459

1. 712487

$-5.348318$

$-6.748916$

$-2.349904$

$-0.933737$

$-0.784177$

$-0.537852$ 


\begin{tabular}{|c|c|c|c|}
\hline $\mathrm{H}$ & -4.547104 & -2.402326 & -1.463130 \\
\hline $\mathrm{C}$ & -3.482517 & -3.847604 & -0.273464 \\
\hline $\mathrm{H}$ & -5.819925 & -1.203423 & 5.040157 \\
\hline C & -4.327490 & -2.755533 & 4.946171 \\
\hline C & -5.605944 & -2.081707 & 4.400877 \\
\hline C & -6.815336 & -3.037451 & 4.497387 \\
\hline C & -0.370007 & 1.927773 & -3.613259 \\
\hline C & 0.235617 & 3.099129 & -3.064861 \\
\hline C & 1.382564 & 3.616723 & -3.692079 \\
\hline $\mathrm{H}$ & 1.855406 & 4.519323 & -3.292078 \\
\hline C & 1.929560 & 2.999969 & -4.826822 \\
\hline $\mathrm{H}$ & 2.821728 & 3.419642 & -5.303698 \\
\hline $\mathrm{C}$ & 1.335434 & 1.842808 & -5.342377 \\
\hline $\mathrm{H}$ & 1.774880 & 1.358332 & -6.221582 \\
\hline C & 0.186728 & 1.275658 & -4.751780 \\
\hline C & -0.391663 & -0.016773 & -5.334661 \\
\hline $\mathrm{H}$ & -1.220740 & -0.345140 & -4.684669 \\
\hline C & -0.398845 & 3.815801 & -1.871136 \\
\hline $\mathrm{H}$ & -0.935569 & 3.048112 & -1.274878 \\
\hline C & 0.183874 & 0.570495 & 4.634035 \\
\hline $\mathrm{H}$ & -0.742073 & 2.137495 & 3.476982 \\
\hline 0 & -0.329770 & 0.069198 & 2.296071 \\
\hline $\mathrm{C}$ & -0.383258 & 1.846579 & 4.468392 \\
\hline $\mathrm{H}$ & -0.935682 & 3.717168 & 5.416200 \\
\hline C & -0.490557 & 2.726258 & 5.560119 \\
\hline $\mathrm{H}$ & -0.116089 & 3.025474 & 7.679493 \\
\hline C & -0.032047 & 2.340188 & 6.829215 \\
\hline $\mathrm{H}$ & 0.893350 & 0.751885 & 7.989139 \\
\hline $\mathrm{C}$ & 0.534693 & 1.064495 & 7.002221 \\
\hline $\mathrm{H}$ & 1.079637 & -0.807677 & 6.055503 \\
\hline C & 0.639181 & 0.187126 & 5.913285 \\
\hline C & 0.339448 & -0.396371 & 3.470883 \\
\hline $\mathrm{H}$ & -0.071317 & -1.383898 & 3.766493 \\
\hline $\mathrm{C}$ & 1.964257 & 0.093021 & 0.546863 \\
\hline Si & 0.116067 & -0.388028 & 0.706910 \\
\hline $\mathrm{C}$ & 2.825421 & -0.578130 & -0.356626 \\
\hline $\mathrm{H}$ & 1.863755 & -2.574964 & 1.474920 \\
\hline $\mathrm{C}$ & 4.156366 & -0.167937 & -0.542175 \\
\hline $\mathrm{H}$ & 1.422910 & -0.548411 & 3.276240 \\
\hline $\mathrm{C}$ & 4.659172 & 0.932531 & 0.172612 \\
\hline $\mathrm{H}$ & 2.451971 & -1.442625 & -0.919745 \\
\hline C & 3.824777 & 1.617408 & 1.072425 \\
\hline $\mathrm{H}$ & 4.803002 & -0.708604 & -1.242697 \\
\hline $\mathrm{C}$ & 2.494672 & 1.201466 & 1.253288 \\
\hline $\mathrm{H}$ & 5.696411 & 1.254714 & 0.029065 \\
\hline $\mathrm{H}$ & 4.212088 & 2.473295 & 1.636955 \\
\hline $\mathrm{H}$ & 1.857306 & 1.742834 & 1.963353 \\
\hline $\mathrm{C}$ & 1.145395 & -4.275820 & 0.365863 \\
\hline $\mathrm{H}$ & 0.371437 & -3.445829 & -1.468851 \\
\hline 0 & 0.059731 & -2.091860 & 0.516356 \\
\hline C & 0.835881 & -4.321329 & -1.005455 \\
\hline $\mathrm{H}$ & 0.859420 & -5.496687 & -2.827763 \\
\hline $\mathrm{C}$ & 1.109003 & -5.475159 & -1.761020 \\
\hline $\mathrm{H}$ & 1.908179 & -7.495686 & -1.742738 \\
\hline $\mathrm{C}$ & 1.696490 & -6.596321 & -1.154548 \\
\hline $\mathrm{H}$ & 2.463037 & -7.429331 & 0.700676 \\
\hline C & 2.007940 & -6.558260 & 0.216473 \\
\hline $\mathrm{H}$ & 1.972913 & -5.386638 & 2.039953 \\
\hline C & 1.732098 & -5.407764 & 0.969762 \\
\hline C & 0.888579 & -3.032319 & 1.203384 \\
\hline $\mathrm{H}$ & 0.402410 & -3.333011 & 2.154212 \\
\hline $\mathrm{H}$ & -5.801156 & -4.548687 & -1.630884 \\
\hline $\mathrm{H}$ & -6.863728 & -3.250508 & -1.018944 \\
\hline $\mathrm{H}$ & -6.213453 & -4.475243 & 0.102155 \\
\hline $\mathrm{H}$ & -4.165919 & 3.650489 & 1.598551 \\
\hline $\mathrm{H}$ & -3.182039 & 2.173829 & 1.374335 \\
\hline $\mathrm{H}$ & -4.082564 & 2.420992 & 2.890808 \\
\hline $\mathrm{H}$ & -6.714697 & 3.536581 & 1.469821 \\
\hline $\mathrm{H}$ & -6.751013 & 2.365418 & 2.809476 \\
\hline $\mathrm{H}$ & -7.546774 & 1.970603 & 1.262958 \\
\hline $\mathrm{H}$ & -3.289245 & -4.516278 & -1.131272 \\
\hline $\mathrm{H}$ & -3.614871 & -4.486044 & 0.618278 \\
\hline $\mathrm{H}$ & -2.581115 & -3.231821 & -0.111100 \\
\hline $\mathrm{H}$ & -4.472268 & -3.078146 & 5.992745 \\
\hline $\mathrm{H}$ & -3.466470 & -2.066174 & 4.917788 \\
\hline $\mathrm{H}$ & -4.062718 & -3.649970 & 4.354473 \\
\hline $\mathrm{H}$ & -6.976241 & -3.357859 & 5.542164 \\
\hline
\end{tabular}

$\begin{array}{rrrr}\mathrm{H} & -6.657848 & -3.947339 & 3.891224 \\ \mathrm{H} & -7.741812 & -2.553667 & 4.142984 \\ \mathrm{H} & 1.061026 & -1.341907 & -4.340437 \\ \mathrm{H} & 1.500333 & -0.926974 & -6.017409 \\ \mathrm{H} & 0.191522 & -2.093629 & -5.706969 \\ \mathrm{H} & -2.240808 & 4.353117 & -2.964174 \\ \mathrm{H} & -0.992032 & 5.625568 & -2.961824 \\ \mathrm{H} & -1.955460 & 5.318242 & -1.489142 \\ \mathrm{H} & -1.756492 & 0.985270 & -6.749322 \\ \mathrm{H} & -1.417136 & -0.724251 & -7.139796 \\ \mathrm{H} & -0.188140 & 0.528274 & -7.457788 \\ \mathrm{H} & 1.400373 & 3.788101 & -0.601416 \\ \mathrm{H} & 0.104517 & 4.878132 & -0.036396 \\ \mathrm{H} & 1.116468 & 5.353233 & -1.413602\end{array}$

\section{$\mathbf{I}^{\mathbf{R}}$}

$-0.777295$

$-2.775237$

$-0.407110$

$-4.497157$

$-2.796153$

$-4.131481$

$-4.446895$

$-4.945333$

$-6.034284$

$-4.081767$

$-1.567075$

$-1.593318$

$-4.623651$

$-5.061765$

$-5.164547$

$-5.383164$

$-5.263039$

$-5.526969$

$-4.830161$

$-4.787553$

$-4.207751$

$-4.093025$

$-6.211398$

2. 465005

0.353590

$-0.323314$

1.081207

$-5.706087$

$-4.367571$

$-3.835292$

$-3.483599$

$-4.885919$

$-7.279435$

$-5.882019$

$-5.980694$

0.812487

1.165175

2.383331

2.665254

3.238698

4.178924

2.889272

3.564838

1.683799

1.274432

0.679940

0.294617

$-0.534075$

4.756852

3.795360

2.330970

4.752885

5.940499

5.961094

8.127346

7.186608

8.148780

7.197900

6.008458
0.437848

0.315341

0.523612

$-0.038498$

0.483350

0.486857

0.595142

0.315798

0.257453

0.208827

0.576543

0.669690

$-1.378432$

$-1.598629$

$-2.630049$

$-0.538974$

0.772814

1.607124

1.048255

2. 496913

2. 506653

3. 455213

3.002151

$-2.645777$

$-2.656987$

3. 354340

4. 311537

$-3.182441$

$-2.576638$

$-2.201930$

$-3.662268$

$-1.641029$

$-1.459822$

$-0.799790$

0.186508

0.620311

1.816696

1.837968

2.746774

0.731683

0.772728

$-0.429682$

$-1.290569$

$-0.511333$

$-1.804835$

$-1.511690$

3.076617

2. 912247

0.324701

1.055244

0.115930

0.897219

1.691131

1.250635

1.314007

1.038840

0.293464

0.466673

$-0.335867$
$-1.520908$

$-2.220884$

$-3.680546$

$-0.409354$

$-3.610819$

$-4.084292$

$-5.123651$

$-2.952605$

$-2.922766$

$-1.824668$

$-4.318066$

$-5.415558$

0.062919

1. 382021

1.738795

2.251195

1.760075

2.421516

0.446971

$-0.048386$

$-0.988730$

0.940782

$-0.378549$

$-3.217194$

$-4.619758$

$-6.488486$

$-4.604261$

$-1.340064$

$-0.857104$

$-1.749023$

$-0.210392$

4.499259

3. 664567

3.672598

4.166271

$-4.419999$

$-5.111495$

$-5.822819$

$-6.366227$

$-5.844782$

$-6.405172$

$-5.140217$

$-5.157501$

$-4.420427$

$-3.714033$

$-2.823207$

$-5.099030$

$-4.389766$

0.051311

$-1.737065$

$-0.078740$

$-1.233300$

$-2.862995$

$-1.859948$

$-1.698475$

$-1.209400$

0.591719

0.075560

1.700213 


\begin{tabular}{|c|c|c|c|}
\hline C & 5.992562 & 0.112561 & 0.698890 \\
\hline C & 3.470771 & -0.054419 & 0.766838 \\
\hline $\mathrm{H}$ & 3.368265 & 0.571611 & 1.677666 \\
\hline $\mathrm{C}$ & 0.792147 & -2.025924 & 1.177645 \\
\hline $\mathrm{Si}$ & 0.737209 & -0.259820 & 0.425448 \\
\hline C & 1.181387 & -2.261061 & 2.519408 \\
\hline $\mathrm{H}$ & -1.472537 & 1.077326 & 1.990903 \\
\hline C & 1.254668 & -3.563024 & 3.043073 \\
\hline $\mathrm{H}$ & 3.542543 & -1.106236 & 1.115408 \\
\hline C & 0.940741 & -4.665953 & 2.230701 \\
\hline $\mathrm{H}$ & 1.432211 & -1.408791 & 3.162588 \\
\hline C & 0.549016 & -4.459929 & 0.897151 \\
\hline $\mathrm{H}$ & 1.558210 & -3.718764 & 4.084758 \\
\hline $\mathrm{C}$ & 0.471952 & -3.153194 & 0.384650 \\
\hline $\mathrm{H}$ & 0.998055 & -5.682112 & 2.636396 \\
\hline $\mathrm{H}$ & 0.299705 & -5.315992 & 0.260009 \\
\hline $\mathrm{H}$ & 0.156377 & -3.007081 & -0.657210 \\
\hline C & -0.467920 & 1.705261 & 3.795691 \\
\hline $\mathrm{H}$ & 1.675309 & 1.915617 & 3.649011 \\
\hline 0 & 0.597224 & 0.767326 & 1.821315 \\
\hline $\mathrm{C}$ & 0.783047 & 2.193529 & 4.217448 \\
\hline $\mathrm{H}$ & 1.861301 & 3.398090 & 5.662788 \\
\hline C & 0.880340 & 3.025883 & 5.346518 \\
\hline $\mathrm{H}$ & -0.194908 & 4.024725 & 6.949431 \\
\hline C & -0.270892 & 3.377219 & 6.069296 \\
\hline $\mathrm{H}$ & -2.429624 & 3.167612 & 6.205229 \\
\hline $\mathrm{C}$ & -1.524182 & 2.894735 & 5.651981 \\
\hline $\mathrm{H}$ & -2.601617 & 1.701350 & 4.196379 \\
\hline $\mathrm{C}$ & -1.620876 & 2.067627 & 4.523009 \\
\hline C & -0.594366 & 0.772102 & 2.601958 \\
\hline $\mathrm{H}$ & -0.822764 & -0.251388 & 2.972500 \\
\hline $\mathrm{C}$ & -0.256332 & 4.732646 & -0.149572 \\
\hline $\mathrm{H}$ & -1.256027 & 5.063198 & -2.047524 \\
\hline O & -0.693753 & 2.644330 & -1.237055 \\
\hline C & -0.822903 & 5.542539 & -1.164709 \\
\hline $\mathrm{H}$ & -1.259901 & 7.564023 & -1.801202 \\
\hline C & -0.822553 & 6.932055 & -1.021817 \\
\hline $\mathrm{H}$ & -0.261879 & 8.611911 & 0.234774 \\
\hline C & -0.259377 & 7.521974 & 0.128250 \\
\hline $\mathrm{H}$ & 0.735024 & 7.189184 & 2.031179 \\
\hline C & 0.302574 & 6.723812 & 1.140175 \\
\hline $\mathrm{H}$ & 0.730275 & 4.693430 & 1.787466 \\
\hline C & 0.303741 & 5.330233 & 1.004720 \\
\hline C & -0.238827 & 3.273571 & -0.252874 \\
\hline $\mathrm{H}$ & 0.208287 & 2.712760 & 0.598814 \\
\hline $\mathrm{H}$ & -5.525802 & -4.020712 & -2.036880 \\
\hline $\mathrm{H}$ & -6.323048 & -2.432308 & -1.863368 \\
\hline $\mathrm{H}$ & -6.295854 & -3.570772 & -0.490141 \\
\hline $\mathrm{H}$ & -3.993874 & 4.460206 & 0.494313 \\
\hline $\mathrm{H}$ & -3.082904 & 3.101980 & 1.210572 \\
\hline $\mathrm{H}$ & -4.667379 & 3.570649 & 1.877097 \\
\hline $\mathrm{H}$ & -6.178517 & 4.034654 & -0.770716 \\
\hline $\mathrm{H}$ & -6.850669 & 3.003211 & 0.522726 \\
\hline $\mathrm{H}$ & -6.699704 & 2.366805 & -1.136664 \\
\hline $\mathrm{H}$ & -3.258325 & -4.455380 & -0.945425 \\
\hline $\mathrm{H}$ & -3.981718 & -4.147729 & 0.647774 \\
\hline $\mathrm{H}$ & -2.525604 & -3.249149 & 0.147712 \\
\hline $\mathrm{H}$ & -5.248075 & -1.764845 & 5.535376 \\
\hline $\mathrm{H}$ & -3.890861 & -1.166174 & 4.541702 \\
\hline $\mathrm{H}$ & -4.757538 & -2.650594 & 4.070463 \\
\hline $\mathrm{H}$ & -7.656112 & -1.592625 & 4.694566 \\
\hline $\mathrm{H}$ & -7.245930 & -2.456420 & 3.189388 \\
\hline $\mathrm{H}$ & -8.011503 & -0.847912 & 3.109990 \\
\hline $\mathrm{H}$ & 3.161576 & -2.048693 & -2.605855 \\
\hline $\mathrm{H}$ & 3.035131 & -3.089532 & -4.052932 \\
\hline $\mathrm{H}$ & 2.101707 & -3.482812 & -2.596493 \\
\hline $\mathrm{H}$ & -0.927863 & 2.503145 & -6.846412 \\
\hline $\mathrm{H}$ & 0.460875 & 3.544273 & -7.243236 \\
\hline $\mathrm{H}$ & -0.977201 & 4.244169 & -6.453759 \\
\hline $\mathrm{H}$ & -0.544834 & -2.099267 & -4.932865 \\
\hline $\mathrm{H}$ & 0.020777 & -3.567685 & -4.090131 \\
\hline $\mathrm{H}$ & 0.889890 & -2.972939 & -5.532626 \\
\hline $\mathrm{H}$ & 1.518458 & 4.140034 & -3.606117 \\
\hline $\mathrm{H}$ & 0.415501 & 5.190439 & -4.539967 \\
\hline $\mathrm{H}$ & 1.904809 & 4.575236 & -5.291003 \\
\hline
\end{tabular}


